\title{
Archytas lu par Simplicius. Un art de la conciliation
}

\author{
Marc-Antoine Gavray \\ Université de Liège_Fonds National de la Recherche Scientifique, Liège, Belgique \\ Marc-Antoine.Gavray@ulg.ac.be*
}

\begin{abstract}
Intent upon harmonizing doctrines of their predecessors, some Neoplatonic commentators are faced with a problem of resolving doctrinal discrepancies so as to restore the $\sigma v \mu \varphi \omega v i \alpha$ in the history of philosophy. This article considers a particular example of this attempt ats harmonization: how Simplicius reconciles Aristotle's Categories with the Neopythagorean doctrine of the Pseudo-Archytas. The chronological inversion introduced by the counterfeiter produces remarkable effects on the late Platonic doctrine about general terms, to the extent that a commentator such as Simplicius works to reduce the dissonance between Archytas' and Aristotle's words.

This paper has three aims: to restore the general grid that Simplicius uses for reading and commenting on Archytas through Aristotle; to identify the exegetical strategies aimed at a doctrinal reconciliation; to consider a specific case, provided by the doctrine of weight, which engenders a new physical theory by Simplicius.
\end{abstract}

\section{Keywords}

authority, exegesis, harmony, Iamblichus, Neoplatonic cursus, weight

À maints égards, le Commentaire de Simplicius sur les Catégories revêt l'apparence d'un palimpseste. Lisant lemme après lemme le texte

\footnotetext{
*) Je tiens à remercier Ph. Hoffmann, les membres de son séminaire, ainsi que les participants du 7th Annual Conference of the International Society for Neoplatonic Studies, Kraków, June 18-21, 2009. Leurs remarques, questions et objections face à différentes parties de cette étude ont contribué à son élaboration progressive.
} 
d'Aristote, il suit à la lettre le Commentaire de Jamblique, qui avait luimême absorbé la contribution de son prédécesseur, Porphyre ${ }^{1}$. Par l'intermédiaire de ce dernier, Simplicius accède aux commentaires péripatéticiens de Boéthos de Sidon et d'Alexandre d'Aphrodise, ainsi qu'aux réponses aux objections soulevées par Plotin à l'encontre d'Aristote. L'enchevêtrement de ces multiples strates alimente l'exégèse, qui mêle les références selon des critères précis afin de brosser un tableau harmonieux et organisé de la tradition grecque. Au sein de l'édifice, chaque élément reçoit une place déterminée et participe à l'interprétation globale du texte aristotélicien. Or, à cette filiation, il reste à ajouter une étape en amont du Stagirite:

Le pythagoricien Archytas, avant même Aristote, avait divisé en dix les genres premiers dans son livre intitulé Sur le Tout; il avait clairement mis en lumière leurs traits caractéristiques au moyen d'exemples, et il avait indiqué l'ordre qu'ils ont les uns par rapport aux autres, les différences spécifiques de chacun et leurs attributs communs et particuliers. Jamblique inséra les textes d'Archytas aux endroits qui convenaient et, comme ils étaient empreints d'une grande concentration intellective, il en donna l'explication; il démontra aussi l'accord d'Aristote avec eux. Que s'il y avait çà et là quelques discordances—mais elles sont peu nombreuses—, il les offrit aux regards des amoureux du savoir, non sans examiner la cause de la discordance: ce qui était fort légitime, puisque Aristote, manifestement, voulait suivre en tout les traces d'Archytas (In Cat., 2, 15-25; tr. Hoffmann).

La paternité pythagoricienne confère à la doctrine des catégories une plus haute autorité, dans la mesure où elle recèle une vérité supérieure. Il incombe dès lors au bon exégète de révéler l'harmonie d'Aristote et de sa source, afin de manifester la doctrine complète des catégories. C'est ce rôle qu'à la suite de Jamblique Simplicius assume au fil du commentaire.

L'histoire se complique dès que l'on se penche sur l'identité de ce philosophe pythagoricien. Contemporain de Platon, avec lequel il aurait entre-

1) Voir Simplicius, Commentaire sur les Catégories d'Aristote (In Cat.), 2, 3-3, 17. Simplicius y affirme: “j'ai pris modèle sur le commentaire de Jamblique, avec tout le soin dont j'étais capable, en le suivant pas à pas et en utilisant fréquemment le texte même de ce philosophe" (3, 2-4; tr. Hoffmann, dans Hadot [1990]). Les citations de Simplicius renvoient aux éditions des Commentaria in Aristotelem Graeca. Sur la filiation des commentateurs néoplatoniciens des Catégories, lire les conclusions de Hoffmann (2000) 375-376. 
tenu une correspondance-apocryphe-, Archytas de Tarente fut un brillant mathématicien, auteur de livres Sur la musique, Sur les Sciences, d'une Harmonique, etc. ${ }^{2}$ Sous son nom subsistent également, de façon fragmentaire, de nombreux ouvrages inauthentiques, datant pour l'essentiel de la période impériale ${ }^{3}$. Le traité auquel se réfere Simplicius dans ce

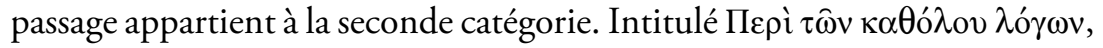
le pseudépigraphe date du $\mathrm{I}^{\mathrm{er}}$ siècle ACN-II ${ }^{\mathrm{e} m e}$ siècle PCN et vise à répondre aux objections formulées contre la doctrine aristotélicienne des catégories ${ }^{4}$. Nourri par les débats de l'époque (Boéthos, Eudore et Andronicos), il rassemble des éléments puisés dans le corpus aristotélicien (Métaphysique, Physique et, bien entendu, Catégories), les enrichit d'emprunts au stoïcisme et les traduit en dialecte dorien, pour se parer d'authenticité. Le jeu d'emboîtement devient alors évident: dans le sillage de Jamblique, Simplicius discerne chez Aristote l'inspiration d'une source pythagoricienne-qui s'avère lui être bien postérieure et entièrement contrefaite.

Palimpseste, c'est bien l'apparence que revêtent les Catégories aux yeux de Simplicius. Dans l'esprit du commentateur, Archytas sert de modèle à Aristote. Par conséquent, l'exégèse du premier doit accompagner celle du second dans la mesure où, loin de se limiter à une pratique scolaire, l'exercice de confrontation aide à révéler la vérité commune et originaire consignée dans ces œuvres majeures. Tout en interprétant les Catégories, Simplicius explique Archytas et enrichit ainsi d'éléments inédits l'exposé des genres premiers. Dans ces conditions, le travail du lecteur moderne consiste à dégager la grille de lecture d'Archytas sous-jacente à celle d'Aristote, dans l'idée qu'un tel examen enrichira nos connaissances des présupposés doctrinaux et de la méthode aux fondements du commentaire de Simplicius. Si, suivant Jamblique, Simplicius adopte une perspective en vertu de laquelle il reconnait au Pythagoricien la division en dix genres,

\footnotetext{
2) Un échange épistolaire entre Platon et Archytas devait offrir la justification de l'influence du pythagorisme sur la doctrine platonicienne. Sur Archytas de Tarente, lire l'étude approfondie de Huffman (2005).

3) Sur la littérature pseudo-pythagoricienne de cette période, Thesleff (1961) et Thesleff (1965).

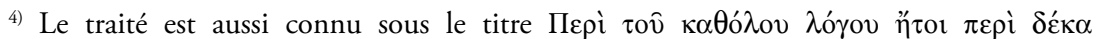
$\kappa \alpha \tau \eta \gamma о \rho ı \hat{v}$. Simplicius est notre source principale, en dehors du seul manuscrit par lequel il a été conservé, le codex Ambrosianus A 92 sup. (gr. 23). Pour l'interprétation, Szlezák (1972). Pour sa chronologie et sa réception, Hoffmann (1980) 308-312.
} 
leurs caractéristiques, leur ordre, leurs différences et leurs attributs, il convient d'examiner la congruence de son hypothèse, car il ne lui échappait pas qu'existaient plusieurs points de désaccord: comment justifier, par exemple, l'absence chez Aristote d'un élément de doctrine, pourtant essentiel, inséré par le faussaire afin de parer une critique rédhibitoire? Aussi rares semblent-elles, ces dissonances représentent les nœuds auxquels s'arriment les efforts pour révéler l'harmonie véritable. Tel que le conçoit du moins Simplicius, le rôle du bon exégète consiste en effet à rétablir l'harmonie, c'est-à-dire à isoler les points de rupture apparents pour restaurer la jonction profonde, inscrite dans la vérité des choses (In Cat., 7, 23-32). La recherche de la convergence manifeste la fécondité du commentarisme dans le néoplatonisme tardif, étant donné qu'elle permet la production de doctrines nouvelles, nées de la rencontre entre des pensées distinctes, voire incompatibles, mais qui, pour un Simplicius, s'ancrent dans la vérité et trouvent leur justification dans les textes mêmes. Étudier ces zones de tâtonnement s'avère dès lors un moyen d'approcher la méthode d'un commentateur, ainsi que la doctrine qui sous-tend son travail, car elles sont un lieu privilégié pour tester la solidité des principes méthodologiques de l'exégèse, dès lors que leur rupture tout comme leur résistance entrainnent certains aménagements philosophiquement pertinents et dignes d'attention.

Cette contribution ne portera ni sur Archytas, ni sur son double, ni même sur les Catégories, mais bien sur leur lecteur, Simplicius5. Elle voudrait esquisser une typologie de l'usage que ce dernier fait d'Archytas et analyser les rapports qu'il conçoit entre l'original (Archytas) et sa copie (Aristote), afin de cerner les procédés mis en œuvre dans la composition, tout autant que les postulats et les apports doctrinaux ${ }^{6}$. À l'aide d'un examen d'ensemble des passages concernés, elle entend dégager les différentes

\footnotetext{
5) Dans la suite de cet article, Archytas servira à désigner le faussaire et son traité, et non le vrai pythagoricien.

6) Deux livres récents ont grandement enrichi nos connaissances sur Simplicius: Baltussen (2008) et Golitsis (2008). Ils nous informent sur ses habitudes de composition, sur ses sources et sur les modalités d'expression de ses doctrines. Toutefois, ils se focalisent sur le Commentaire à la Physique. C'est en vue d'esquisser un pendant à ces travaux pour le Commentaire sur les Catégories que cette étude se penchera sur l'utilisation d'Archytas par Simplicius dans son exégèse des Catégories.
} 
stratégies d'utilisation avant, dans une dernière partie, de proposer une illustration des innovations doctrinales issues des procédés identifiés.

\section{Le questionnaire néoplatonicien}

Approcher Archytas se révèle un exercice délicat. D’une part, Simplicius n'en propose pas de commentaire lemmatique mais l'intègre à son exégèse d'Aristote. D'autre part, les passages à examiner se trouvent disséminés à travers le Commentaire sur les Catégories, de la deuxième page à l'avantdernier paragraphe: Archytas retient l'attention de Simplicius en une centaine d'endroits. Un expédient pour affronter cette double difficulté consisterait à appliquer un principe codifié de l'exégèse néoplatonicienne tardive, qui devrait fournir une perspective unifiée sur le Pythagoricien, respectueuse des méthodes en cours chez ces commentateurs.

Afin de tracer les lignes de conduite pour l'interprétation, un Simplicius adresse à l'œuvre en présence six questions préalables, "à savoir le but, l'utilité, la raison d'être du titre, l'ordre de lecture, s'il s'agit d'un livre authentique du philosophe, la division en chapitres". Il en ajoute une septième: "peut-être n'est-il pas non plus absurde de chercher de quelle partie de la philosophie il relève ${ }^{7}$." Les réponses dessinent les contours de l'interprétation et définissent le cadre général à l'intérieur duquel évolue l'exégèse. Elles énoncent des précautions, établissent la valeur des informations et exposent la manière de rendre compte des difficultés.

Afin de rassembler les éléments épars et de saisir l'optique adoptée à l'égard du Pythagoricien-en lui-même mais aussi dans sa relation aux Catégories_-, je voudrais me livrer à un jeu de reconstitution de ce qu'aurait pu être ce questionnaire au sujet d'Archytas. Aussi artificiel que paraisse cet exercice, il pourrait s'avérer riche d'un intérêt heuristique-bien qu'il ne se trouve nulle part comme tel chez Simplicius, pour ce qui regarde Archytas. Identifier le but du traité, en évaluer l'utilité, en comprendre l'ordre ou discerner la partie de la philosophie qu'il vise, c'est en effet reconstituer une grille d'interprétation à laquelle Simplicius devait (aurait pu) se conformer. Par conséquent, c'est déjà engager l'interprétation du pseudo-Archytas, mais aussi la façon dont il convient de le lire par rapport à Aristote. Dans

7) In Cat., 8, 10-13. Simplicius y répond pour les Catégories en In Cat., 8, 9-20, 12; pour la Physique en In Phys., 1, 3-6, 30. 
un second temps, il deviendra commode d'évaluer la fidélité de Simplicius vis-à-vis de ses principes exégétiques.

\section{Le but}

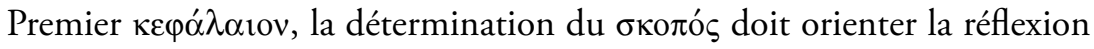
et éviter sa dispersion en rapportant toutes les explications à un principe unique. Concernant nos traités, elle revient à définir la notion de catégorie et l'intention poursuivie dans son étude. Le traité Sur les notions universelles porte donc sur les genres les plus élevés, dans la mesure où les universels sont toujours attribués de réalités inférieures, sans jamais être eux-mêmes substrats. Il sert en cela, affirme Simplicius, le même objectif que les Catégories (17, 25-28).

Selon le бколó $\varsigma$ formulé par Porphyre et repris par Simplicius, les Catégories portent "sur les mots simples et premiers qui désignent les réalités premières et généralissimes au moyen des notions simples et premières (13, 19-21)." En d'autres termes, les catégories ne s'identifient pas aux êtres en tant que tels, mais aux mots qui les signifient en tant qu'ils les signifient (16, 20-21). Le traité d'Aristote concerne les mots dans leur relation étroite de signification avec les réalités ${ }^{8}$. La complexité de cette définition résulte de la différenciation entre mot, réalité et notion, qui survient après un état d'unité originelle. Dans la doctrine néoplatonicienne tardive, être et notion se confondent d'abord au sein de l'Intellect, au sens où l'intellection possède tant les êtres que leurs notions, au moyen d'une union sans distinction. Par conséquent, ce niveau consacre l'inutilité du langage. Or, à partir du moment où l'âme s'écarte de l'Intellect, elle en vient à

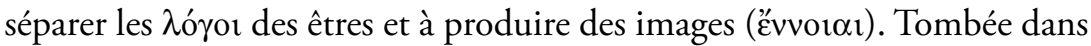
le devenir, elle ne connaît d'autre solution pour se souvenir et exprimer la vérité que d'instaurer un langage en relation étroite avec les notions des réalités qu'elle cherche à désigner' ${ }^{9}$. C'est ce niveau de l'institution du langage dans sa relation aux choses que les Catégories prendrait pour бколós.

Si Simplicius attribue aux deux traités un бколó $\varsigma$ identique, certaines explications postérieures conduisent à nuancer cet accord. L'attention

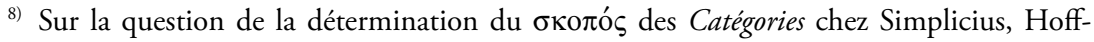
mann (1987).

9) L'origine de l'instauration nécessaire du langage est expliquée en In Cat., 12, 16-32.
} 
d'Archytas semble ainsi davantage porter sur les êtres eux-mêmes, tandis qu'il laisse l'expression au second plan. Au premier chef, il expose comment sont les choses, sans se contenter de comment elles sont dites. En effet, il

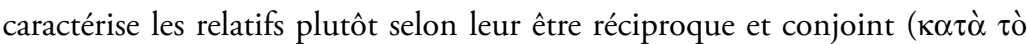

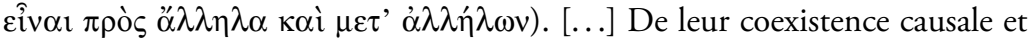

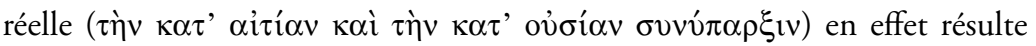
leur coexistence linguistique ( $\dot{\eta} \tau$ ov̂ $\lambda \varepsilon ́ \gamma \varepsilon \sigma \theta \alpha$ ). Car en tant qu'une chose est, elle est aussi dite, et parce qu'elle est, elle est dite; mais ce n'est pas parce qu'elle est dite, qu'elle est. En tant qu'une chose est de telle nature, elle amène la particularité du genre; alors qu'en tant qu'elle est seulement dite, personne ne pourra exposer sa distinction générique, de telle sorte que la réciprocité

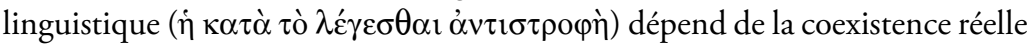

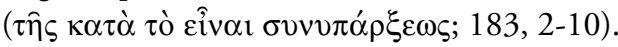

Bien qu'il ne s'exprime en rien moins précisément qu'Aristote, pourquoi Archytas a-t-il omis cette particularité des relatifs: se dire dans le rapport à son

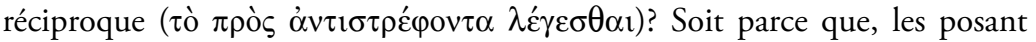

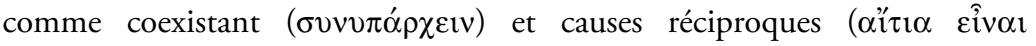
$\dot{\alpha} \lambda \lambda \eta \dot{\eta} \lambda \omega v)$, il a admis leur réciprocité réelle (

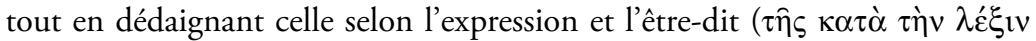

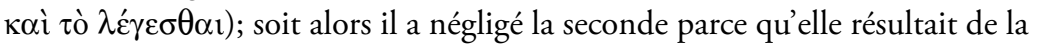
première $(189,12-16)$.

Selon Simplicius, Archytas ne se satisfait pas de considérations sémantiques mais privilégie les déterminations ontologiques au détriment des aspects linguistiques. L'exemple illustre son point de vue, qui consiste à étudier les relatifs non pas en tant qu'ils désignent des termes que l'on dit en relation l'un par rapport à l'autre, mais en tant qu'ils sont des réalités en relation effective l'une avec l'autre, car seule l'existence révèle les déterminations génériques en vertu desquelles deux termes s'opposent. Leur coexistence précède leur réciprocité linguistique. Chez Archytas, l'enquête prend un tour ontologique en vertu duquel l'être prime sur l'être-dit, se situant à un niveau plus élevé, qui s'enquiert moins du $\lambda$ ó $\gamma$ os que des $\pi \rho \alpha ́ \gamma \mu \alpha \tau \alpha-$ de l'être qu'il signifie et de la notion qu'il recouvre. Selon l'usage pythagoricien repris par Platon, il se penche sur les réalités naturelles en tant qu'elles participent aux surnaturelles: il traite d'un plan du réel antérieur à la discrimination achevée qui a abouti à l'institution du langage (6, 27-30). 
Archytas se montre aussi davantage attentif à l'unité qu'à la multiplicité: il poursuit l'unité et se satisfait de trouver un genre, car il vise ce qui assure l'identité de la classe. Dans l'analyse des genres, il s'attèle moins à détailler leurs divisions qu’à énoncer la propriété générique qui leur confère l'unité. Là où, en vue d'atteindre l'unité du genre, Aristote part de la multiplicité spécifique qui nous est immédiatement accessible, Archytas secondarise la division de l'un en multiple et nie qu'une pluralité puisse révéler l'unité. À ses yeux, la pluralité doit toujours s'observer $\pi \varepsilon p i ̀$ tò év, c'est-à-dire eu égard à l'unité dont elle dépend et à laquelle elle s'avère postérieure, tant dans l'ordre logique que méthodologique $(240,20-29 ; 272,2-6)$. Les deux auteurs adoptent des points de vue opposés que Simplicius juge pourtant complémentaires. Pour les concilier, il lui suffirait de les hiérarchiser, aussi bien sous l'aspect exégétique que gnoséologique. L'apprenti philosophe devrait successivement s'atteler à maîtriser ces regards, afin de s'élever dans la connaissance: d'abord passer du multiple à l'un, à partir du monde qui lui est familier, pour ensuite atteindre le privilège de l'unité sur la multiplicité. Il arriverait alors au point de vue premier dans l'ordre de la connaissance en soi, mais second dans celui de la connaissance pour nous.

La perspective unitaire du Pythagoricien se manifeste également par un privilège accordé à la pureté des genres. Archytas s'occupe de ce qui caractérise le genre comme tel et qui en assure l'identité, sans se soucier des

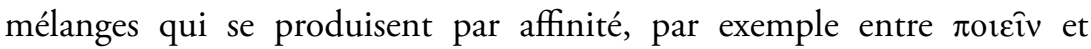
$\pi \alpha ́ \sigma \chi \varepsilon ı v$. À nouveau, il vise à saisir le genre dans l'intégrité de son unité, en le purifiant de tout résidu extérieur: il conçoit l'agir et le pâtir en tant que tels, et non en ce qu'ils conservent les traces d'un mélange réciproque ${ }^{10}$. Au-delà des aspects méthodologiques, cette perspective rend possible l'élaboration d'une cosmologie fondée sur un modèle démiurgique. Envisagés

10) En In Cat., 315, 1-9, Simplicius rejette l'hypothèse de Jamblique selon laquelle l'Intellect serait défini comme passion pure, car l'Intellect recèle toujours un résidu actif en éveil, comme toute forme de connaissance. Selon Simplicius, qui prétend seulement restituer une vérité déjà exprimée avec concision, Archytas s'astreint à une recherche de l'identité du genre. Pour cette raison, il parle d'actions et de passions pures, et non en tant qu'elles s'entremêlent. Il propose donc pour l'agir l'íøıv suivant: posséder la cause de son mouvement en soi-même, et pour le pâtir: avoir sa cause en un autre. Lidée de pureté des catégories implique de bien saisir la signification de $\dot{\varepsilon} v \alpha \hat{\tau} \tau \hat{̣}$. Dans ce cas, la locution équivaut à $\dot{\varepsilon} v$ ö $\lambda \omega$ : c'est en totalité qu'un acteur doit être acteur, le patient patient, autrement dit une partie ne peut pas être cause ou agent, l'autre causée ou patient. 
dans leur pureté, le roteîv désigne le dieu (ce par quoi agit tout ce qui agit

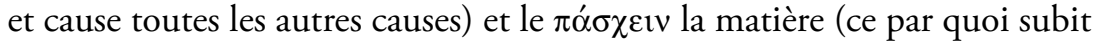
tout ce qui subit), tandis que les éléments résultent de leur mélange. Ces

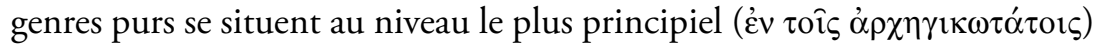
et engendrent toutes les substances qui nous entourent: sous l'effet de l'action du divin, le Ě $\chi \varepsilon ı v$ arrive à la matière et donne naissance à la substance composée $(334,20-27)$. Il apparaît qu'Archytas n'identifie pas tout simplement les genres premiers à des catégories, mais qu'il établit la façon dont ces termes premiers et universels valent aussi à titre de réalités dotées du rang de principes constitutifs du monde. Si, sur ce point, la comparaison avec Aristote s'avère délicate, puisqu'à la différence d'Archytas il n'offre aucun traitement détaillé des genres mineurs (auxquels appartiennent le

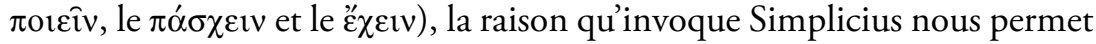
de mesurer l'écart conçu par notre commentateur entre les buts respectifs de ces deux auteurs: Aristote restreint l'exposé relatif à ces genres, parce qu'aucun développement ni aucune théorie avancée par Archytas ne conviendrait à un traité de logique $(300,19-25)$. Il appert que là où l'un s'autorise à s'enquérir de la constitution du monde, l'autre se tait délibérément, eu égard au statut de son exposé.

En conclusion, si de prime abord le traité Sur les notions universelles reçoit de Simplicius le même oкotós que les Catégories, au fil des analyses son point de vue s'avère plus théorétique à maints égards. Il privilégierait une perspective unitaire distincte de la primauté qu'Aristote reconnaît à la multiplicité et recentrerait le propos par le rappel que les réalités se trouvent premières par rapport au langage. Il en résulte qu'il viserait à dresser un exposé de l'ontologie pythagoricienne plutôt qu'à fournir une logique trop détachée des réalités que ses termes désigneraient. Il accéderait à un niveau plus élevé dans la compréhension de la signification originaire des mots simples et premiers, qui sont les plus génériques de tous, et introduirait à un état plus unifié de la langue, dans son noyau fondamental, qui élève l'âme vers de plus hautes connaissances et la conduit vers le premier principe. Bref, il renouerait avec une étape de la procession antérieure à cette séparation achevée qui a mené à l'institution du langage.

\section{L'utilité}

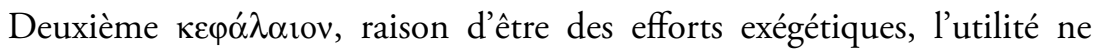
paraît pas indépendante du but. "Il faut savoir qu'on n'a pas toujours 
besoin d'expliquer tous ces points. Souvent en effet l'utilité apparaît en même temps que le but" (8, 31-9, 1; tr. Hoffmann). Si les Catégories enseignent sur les mots simples qui engendrent les expressions complexes d'où surgiront les syllogismes, elles s'avèrent utiles à titre d'introduction, tant pour la philosophie dans son ensemble que pour la seule étude de la logique. Dans la mesure où il partagerait un but similaire, le traité d'Archytas devrait avoir une même utilité.

Les précisions apportées à la définition du $\sigma \kappa о \pi$ ó incitent à quelques nuances. Le traité Sur les notions universelles a pour fonction de nous instruire sur les êtres premiers envisagés du point de vue supérieur de leur unité, afin d'exercer notre âme à les saisir plus directement. Il constitue une étape plus avancée dans l'élévation vers le principe premier, qui nous initie au processus qui a incité à l'institution du langage.

\section{La justification du titre}

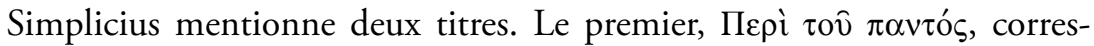

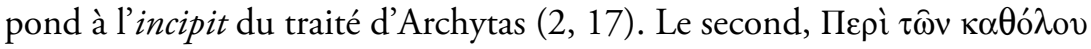
$\lambda$ ó $\gamma \omega v$, fait écho au titre transmis par le seul manuscrit conservé $(13,23$; 17, 26). Dans l'optique de Simplicius, ce dernier intitulé s'avère plus adéquat car il résulte d'un effort de synthèse qui annonce le contenu de l'ouvrage, alors que le premier procède simplement de l'ouverture du texte. Or le titre idoine doit confirmer le but: traitant des genres généralissimes et premiers qui forment le noyau du langage, Archytas aurait choisi l'intitulé qui rendait le mieux raison de son objet, les notions universelles. De plus, ce titre s'avère plus approprié que celui adopté par Aristote, qui aurait retenu Catégories à la seule fin d'éviter la confusion avec Archytas (18, 9-11). Cette contrainte de clarté aurait ainsi conduit Aristote à répéter le geste initial des onomatothètes: en philosophe véritable, il aurait attribué au terme 'catégorie' une signification plus convenable et plus conforme à la nature de son objet par le biais d'une restriction de l'usage ordinaire, pour en limiter le sens à l'idée de prédication $(17,29-18,6)$.

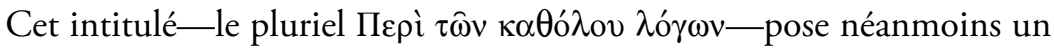

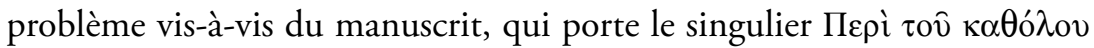
$\lambda$ óyov. Szlezák (1972; 94-95) suggère de voir dans cette variante une confusion, sans la moindre incidence, entre la forme dorienne du génitif singulier en - $\omega$ et le génitif pluriel en - $\omega v$. L'argument serait imparable s'il ne fallait tenir compte de deux passages où Simplicius emploie le singulier 
tout en l'agrémentant d'un déterminatif au pluriel: $\tau o \hat{v} \tau \hat{\omega} v \kappa \alpha \tau \eta \gamma o \rho i \hat{v} v$

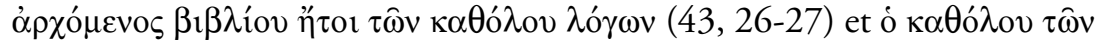

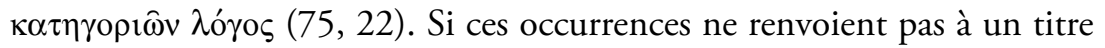
à proprement parler, elles mettent en balance le discours universel (au singulier) et ses objets (au pluriel: les catégories ou notions universelles), faisant plutôt porter l'accent sur ces derniers. Parler de discours universel (au singulier) implique de se positionner résolument sur le plan logique et discursif, dans la mesure où l'attention se focaliserait sur le discours capable de rendre compte de toutes les réalités. En revanche, l'insistance de Simplicius sur le pluriel se révèle riche de sens: elle lui permet d'insister sur la multiplicité des dix termes universels qui servent à désigner les réalités, ouvrant de ce fait la possibilité de souligner la perspective ontologique que notre commentateur attribue à Archytas, au détriment de l'aspect logique.

Formé sur l'incipit, l'autre titre soulève également une question, dont la solution résonne avec le premier problème. Si le traité commençait de la

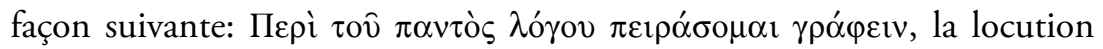

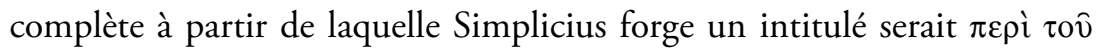

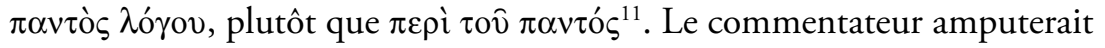

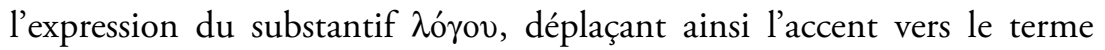
$\pi \alpha \nu \tau o ́ \varsigma$ qui, dans ces conditions, n'occupe plus une position d'adjectif épithète mais tient lieu de substantif. Au lieu d'un traité Sur le discours total, Simplicius citerait un écrit Sur le Tout (en raison de la similitude entre le masculin et le neutre). Or l'incipit du manuscrit renvoie à l'idée d'expres-

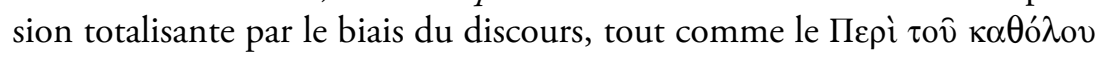
$\lambda$ ó logique, traitant du discours total en mesure de tout désigner. En revanche, la version de Simplicius altère le propos, vu qu'il l'inscrit dans la perspective d'une étude sur la totalité des choses (cf. 128, 27-28: ce changement remonterait à Jamblique).

La distorsion chronologique induite par la croyance en l'authenticité du pseudépigraphe crée une inversion des rapports de référence. Elle

11) Cette première phrase manque dans les fragments transmis par Simplicius, mais elle apparaît dans le manuscrit parallèle. Simplicius emploie également cet intitulé dans l'In

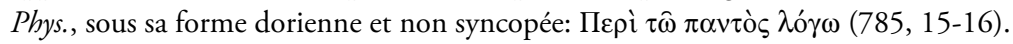

12) Szlezák (1972) 97-98. 
conduit Simplicius à souligner les mérites de chacun de ses deux auteurs. Amené à se positionner par rapport à Archytas, Aristote a réalisé un geste philosophique d'institution du langage. Quant à Archytas, il a clairement ciblé l'objet de son traité. Accentuée par les modifications apportées au titre de l'œuvre du Pythagoricien, cette différence reproduit celle de niveaux que nous avons déjà rencontrée: Archytas écrit sur les termes universels eux-mêmes, ces réalités plus principielles, tandis qu'Aristote les étudie dans leur rapport avec les termes qui leur sont inférieurs et dont ils sont prédiqués.

\section{La place dans l'ordre de lecture}

Simplicius ne dit rien au sujet de la place Des notions universelles car, comme tous les écrits pythagoriciens, ce traité n'appartenait pas au cursus studiorum proprement dit et ne faisait pas l'objet d'une lecture autonome.

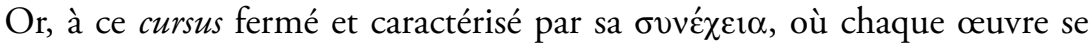
voyait attribuer un temps adapté à son étude, il arrivait de greffer quelques éléments extérieurs. Dans ces circonstances, quel moment Simplicius aurait-il jugé opportun pour la lecture d'Archytas? Pour formuler la question autrement, que penser de la mise en parallèle que propose Simplicius entre les Catégories et Les notions universelles?

Pour toute information sur l'ordre, Simplicius signale que la lecture Des notions universelles doit précéder celle d'un autre traité, Sur les opposés—seul autre texte d'Archytas auquel se réfère le commentateur dans l'In Categorias $^{13}$. Si Archytas a négligé de traiter l'homonymie, sur laquelle s'ouvrent les Catégories, il a aussi discuté séparément les problèmes concernant les opposés, étant donné qu'ils annoncent la formation d'expressions

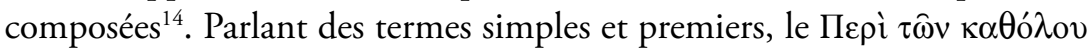

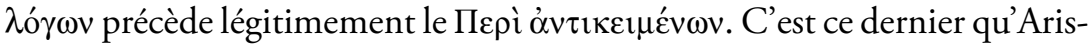
tote aurait transposé à la fin des Catégories:

13) Dans le Commentaire sur la Physique, Simplicius cite deux témoignages empruntés à Eudème. Ceux-ci ont été attribués au véritable Archytas et classés respectivement A 23 (= In Phys., 431, 6-16) et A 24 (= In Phys., 467, 26-468, 3) dans le recueil Diels-Kranz. Sans titre, ces deux textes appartiennent clairement à d'autres ouvrages que les deux mentionnés dans l'In Categorias.

14) Pour le pythagorisme, les noms ont une origine naturelle et ne désignent dès lors qu'une seule chose $(40,5-8)$. 
Aristote a manifestement transcrit les passages sur les opposés à partir du livre d'Archytas intitulé Sur les opposés, que ce dernier n'a pas joint à son texte sur les genres, mais qu'il a jugé digne d'un traité séparé (382, 7-10).

Qu'en aurait-il été de ces traités au sein du programme, par rapport aux écrits aristotéliciens? Les notions universelles auraient vraisemblablement dû succéder aux Catégories. Le premier traité contient en effet une doctrine plus scientifique adressée à des philosophes confirmés, et non à des débutants. Sa brachylogie recèle une théorie d'un ordre supérieur, exprimée sur un ton apodictique, qui requiert un déploiement de la part de l'exégète ${ }^{15}$. Avec Simplicius,

il faut établir à propos des écrits archytéens que rien n'y trahit la recherche ni

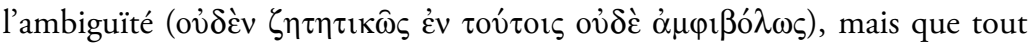
est avancé de façon catégorique ( $\dot{\alpha} \pi \circ \varphi \alpha v \tau \tau \kappa \hat{\omega} \varsigma)$, avec le courage propre à la science, et procède de la manière la plus nécessaire $\left(\delta \mathrm{r}^{\prime} \alpha \hat{v} \tau \hat{\omega} v \mathrm{v} \tilde{\omega} \mathrm{v}\right.$

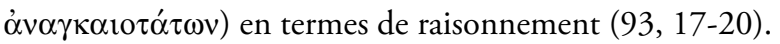

À l'inverse, Aristote écrit sur le mode de la recherche de la vérité ( $\delta$ ì̀

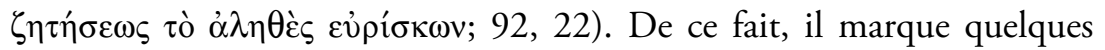
hésitations et affiche une certaine prudence (au moyen d'expressions telles que ठокєî, 189, 27-29). En outre, il passe brièvement sur ce qui relève des notions communes, en raison de leur évidence, là où Archytas présente un exposé complet et détaillé (300, 15-19; cf. Cat., 11b14-15). Or, pour emboîter le pas à Simplicius, nous devrions nous demander pourquoi Aristote persiste à hésiter, au lieu de recourir aux doctrines d'Archytas, dans leur affirmation, alors qu'il les connaît par ailleurs:

Bien qu'il connaisse la division de la substance dans les termes d'Archytas, comment donc Aristote ne l'a-t-il pas utilisée ici $(91,28-30)^{16}$ ?

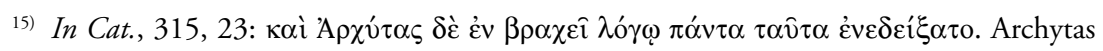
dit d'une façon plus concise ce que Jamblique a exprimé avec ambages. Cf 290, 11-12:

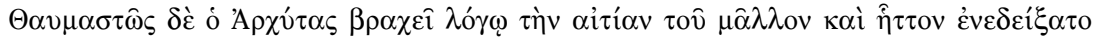

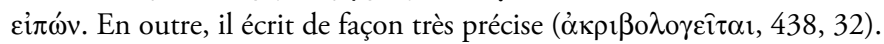

16) Simplicius fait allusion à la division de l'ov̉ $\sigma i ́ \alpha$ en matière, forme et composé des deux, qui se retrouve dans la Métaphysique (par exemple Z 10, 1034b34-1035a4; H 1, 1042a26-31). 
Simplicius juge que les doctrines d'Archytas ne cadrent pas avec un traité logique à finalité introductive. Aristote obéirait aux critères pédagogiques qui résultent de son objet: partir des substances composées, comme il le fait, implique de se focaliser sur les notions communes et sur l'usage habituel des mots, ainsi que de souligner la multiplicité des substrats avec laquelle le lecteur débutant est plus familier, afin de progresser vers la vérité. Il tiendrait des propos adaptés à une introduction à la philosophie, alors qu'Archytas se prête à davantage de théorie et de précision

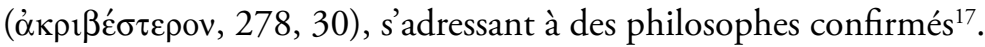

Or, à une occasion, Simplicius attribue une valeur introductive au traité d'Archytas, afin de justifier pourquoi il évite d'exposer des aspects plus élaborés de la doctrine pythagoricienne-à savoir la distinction de l'espèce la plus élevée du $\pi$ oteîv, le théorétique au sens propre, dont l'activité relève des actes d'intellection. Selon Simplicius, Archytas se serait tu "en raison de l'inadaptation d'une telle théorie à une introduction (317, 27-29)". Que conclure d'un tel revirement? Trois hypothèses semblent possibles:

- Simplicius se méprend et transfere à Archytas le caractère isagogique des Catégories. Aussi plausible soit-elle, cette explication est confrontée

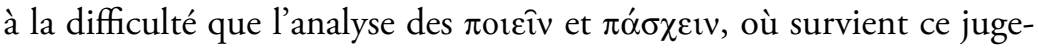
ment, s'éloigne considérablement de la lettre des Catégories, muettes sur le sujet, pour emboîter le pas à Archytas.

- Il nous instruit à propos du moment où greffer ce traité sur le programme d'études: la lecture Des notions universelles devrait venir compléter les écrits du premier cycle, au moment des Catégories. Toutefois, la vérité pythagoricienne n'est-elle pas supposée survenir à un stade avancé du cursus, tant elle dépasse le niveau de la logique?

- Le sens d'introduction n'est ni pédagogique ni chronologique, mais logique: Archytas fournirait l'enseignement complet sur les termes simples qui formeront les expressions composées à la base de la discursivité, c'est-à-dire de l'activité de l'âme qui n'a pas encore atteint l'union intellective avec l'objet. Il en résulte que les précisions relatives aux intelligibles ne s'y trouvent pas nécessaires, puisqu'il s'agit pour l'âme à

17) Le caractère introductif des Catégories est rappelé en 13, 27-28; 110, 24-25; 264, 1-4; 295, 6-10; 387, 23-24; 411, 5-6; 418, 24-28; 426, 34-427, 2. 
ce stade de s'élever à un degré plus haut de la réalité, jusqu’à ces choses mêmes ${ }^{18}$.

Pour Simplicius, le traité Sur les notions universelles d'Archytas décrit un niveau plus élevé du réel que les Catégories. Il possède plus de scientificité et de précision, de sorte qu'il ne convient pas à des débutants. Il offre une brillante introduction à l'ontologie pythagoricienne et sert de preuve dans l'examen des Catégories. Toutefois, sa pleine compréhension requiert des connaissances d'ordre supérieur, acquises lors d'une étape postérieure du cursus. Bref, il peut sous-tendre un commentaire des Catégories, mais pas occuper la même place.

\section{L'authenticité}

Au sein d'un néoplatonisme aux accents néopythagorisants tel que celui de Jamblique, il ne pouvait être question de révoquer l'autorité d'un Pythagoricien de la renommée d'Archytas. Il fournissait un argument supplémentaire en faveur de la thèse de la supériorité de cette vérité. Dès l'Antiquité, des doutes se levèrent pourtant quant à l'authenticité du pseudépigraphe. Au IV ème siècle, en réaction à la position de Jamblique, Thémistius argua qu'il fallait voir en lui un péripatéticien qui avait voulu conférer à une œuvre nouvelle l'ascendant d'un nom ancien et prestigieux ${ }^{19}$. C'est sans doute lui que Simplicius vise lorsqu'il réfute la thèse de certains ( $\tau \iota v \varepsilon ́ \varsigma$ ) qui voyaient dans la définition archytéenne du temps une combinaison d'éléments aristotéliciens et stoïciens (In Cat., $350,13-18)^{20}$. Cette position semble être restée isolée.

Dans la lignée de Jamblique, Simplicius ne doute nullement de l'authenticité du traité, allant jusqu'à défendre son antériorité et son originalité par rapport aux doctrines d'Aristote.

18) De la même façon, le Timée de Platon occupe une des marches les plus élevées de la hiérarchie des dialogues, bien qu'il ne traite pas directement des premiers principes (à la différence du Parménide): il s'exprime sur son sujet avec un plus haut degré de vérité.

19) Le témoignage de ce débat a été conservé par Boèce, In Cat., I, 162A Migne. Cf. Szlezák (1972), 30 et 91-92.

20) Sur l'authenticité de ce traité et ses mises en doute, Hoffmann (1980) 310-312. 


\section{La division en chapitres}

Dernier кєфó $\lambda \alpha \_$, l'explication de la division d'une œuvre en chapitres vise à la mise en lumière de ses articulations. À l'instar du médecin, l'exégète découpe le texte $\kappa \alpha \tau^{\prime} \alpha^{\prime} \rho \rho \alpha$, en respectant son anatomie $(8,25-30)^{21}$. De cette façon, il découvre l'utilité de chaque partie au sein du tout, afin de procurer une connaissance plus précise du composé par la mise à nu des éléments simples. Il souligne comment chacune differre du tout, tout en contribuant à l'objectif global. À partir du moment où l'œuvre étudiée apparait comme un organisme qui adjuge aux éléments une place déterminée, les relations d'interdépendance, de succession ou de complémentarité deviennent un objet d'étude en soi. S'agissant de catégories, il convient de récuser l'idée d'une formulation rhapsodique pour, au contraire, souligner la nécessité des enchaînements de leur énoncé et de leur examen. Dans cette perspective, les questions d'ordre se voient investies d'une importance qui peut surprendre le lecteur moderne.

Tandis que les Catégories comptent trois parties, le livre d'Archytas est d'un seul tenant. Il n'envisage pas la question des homonymes et renvoie celle des opposés à un traité séparé. En revanche, il accorde aux yeux de Simplicius un soin particulier à expliciter ses articulations. À la différence d'Aristote, Archytas s'avère attentif à fournir un ordre aux notions universelles, auquel il demeure fidèle à de rares exceptions près. Non content d'en exprimer les propriétés, il insiste sur les relations qui les lient et assortit cet ordre d'une raison ${ }^{22}$. Par cette attention, il rejoint l'ambition des commentateurs néoplatoniciens de réaliser une déduction des catégories en justifiant la place de chacune. Démontrer l'emboîtement de ces termes et adopter une $\tau \alpha \dot{\alpha} \xi_{1 \zeta}$ définie, c'est en effet révéler une conception du monde et des réalités que recèlent les notions simples-même si, dans les faits, ces transitions se résument souvent à de simples adverbes conjonctifs (عì $\alpha$, हैं $\pi \varepsilon i \tau \alpha$, etc.).

Or c'est au sujet de l'ordre que surgissent le plus grand nombre de divergences entre Archytas et Aristote, dont le seul point commun revient

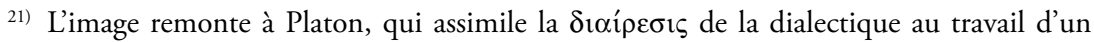
boucher sacrificiel ou d'un médecin qui opère les coupes en respectant les articulations

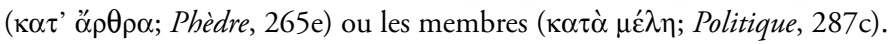

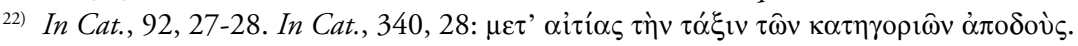


en définitive à placer l'ov́oí en premier lieu ${ }^{23}$. Un tableau synoptique permet d'observer ces variations:

\begin{tabular}{|c|c|c|c|c|}
\hline \multicolumn{2}{|c|}{ Aristote } & \multicolumn{3}{|c|}{ Archytas } \\
\hline Liste initiale & Exposé & $\begin{array}{c}\text { Liste de } \\
\text { Simplicius }\end{array}$ & $\begin{array}{l}\text { Ordre de } \\
\text { Simplicius }\end{array}$ & $\begin{array}{c}\text { Liste de } \\
\text { l'Ambrosianus }\end{array}$ \\
\hline 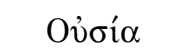 & 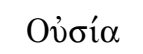 & 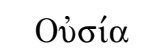 & 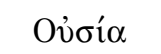 & 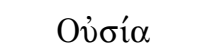 \\
\hline Побóv & Побóv & Пoró $\tau \eta \varsigma$ & 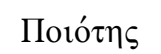 & Пoró $\eta \varsigma$ \\
\hline Пoróv & Прó $\tau_{\imath}$ & 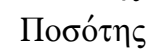 & Побó & 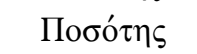 \\
\hline Прó $\varsigma \tau$ & חotóv & Прós $\tau_{1}$ & $\Sigma \chi \varepsilon \dot{\sigma} \sigma \varsigma$ & 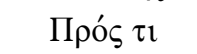 \\
\hline Пov́ & Повєิ̂ิ & Повєิิv & 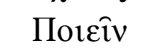 & Повєิิv \\
\hline По $\tau \varepsilon^{\prime}$ & 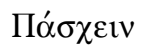 & 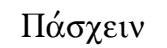 & 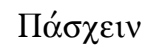 & $\Pi \alpha ́ \sigma \chi \varepsilon \imath \nu$ \\
\hline 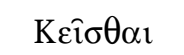 & 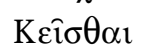 & 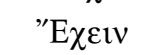 & 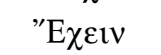 & 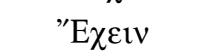 \\
\hline 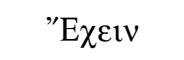 & По $\tau \varepsilon$ & 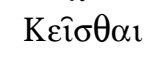 & Тóлоs & 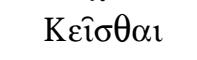 \\
\hline Повєิิv & Пov́ & Пov́ & Xpóvos & Пov́ \\
\hline 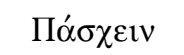 & 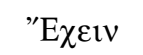 & По $\tau \varepsilon^{\prime}$ & 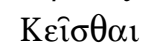 & По $\tau \varepsilon^{\prime}$ \\
\hline
\end{tabular}

Comme le souligne Simplicius, Aristote adopte un ordre différent dans sa liste initiale (Cat., 1 b 25-27) et au fil de l'examen. Un constat analogue vaut pour Archytas: entre l'ordre qu'il est possible de reconstruire, non sans peine, à partir des informations glanées chez Simplicius et la liste qui devait figurer au début du traité-que l'information provienne de Simplicius ou du manuscrit Ambrosianus (qui présentent des listes similaires)—, surviennent de menues variations. Elles concernent les dénominations (Archytas emploie tantôt les substantifs, tantôt les interrogatifs) et l'ordre des catégories de fin de liste. Il appert en outre qu'entre Archytas et Aristote, les points communs sont rares, même sur le plan des premières catégories. Or, comme y insiste Simplicius, la tâche du commentateur, et de toute personne intellectuellement curieuse, implique de ne pas se focaliser sur le désaccord mais d'examiner pourquoi l'accord qui règne généralement entre les deux auteurs se trouve subitement rompu, afin de déterminer les intentions de chacun (Cf. In Cat., 7, 23-32; 121, 18-20). Dans ces conditions,

23) Simplicius ne s'étend pas toujours sur les variations taxinomiques et se contente parfois de les signaler, sans fournir d'explication. C'est le cas en 296, 4-8. 
comment Simplicius parvient-il à maintenir l'importance et la justification de la $\tau \alpha \dot{\alpha} \xi ı$ ?

La première difficulté survient dès la deuxième catégorie, puisqu'Aristote traite de la quantité, Archytas de la qualité. À l'examen, ce premier écart semble résulter de deux conceptions de l’ov̉oía. Autrement dit, une variation d'ordre se produit dès la deuxième étape car la première s'avère moins identique qu'il n'y paraissait.

Après la substance, Archytas a placé le quel, justifiant cet ordre de la façon suivante: "Leur ordre est le suivant: la substance vient la première du fait qu'elle seule subsiste aux autres et peut être pensée par elle-même, alors que les autres ne le peuvent pas sans elle. C'est en effet soit relativement à elle soit à elle qu'elles sont prédiquées dans un substrat (121, 13-17; cf. 206, 15-16).”

Archytas semble supposer que ce qui est intelligible par soi, qui fournit la complétion de façon réelle à tous les genres, qui est présent dans tous sans morcellement et qui est participé par eux préexiste et attribue l'ordre aux genres en fonction de leur parenté avec lui. Il place en effet la substance avant les autres ${ }^{24}$ parce que, subsistant à tous les autres, elle leur fournit l'être à partir d'elle-même, de même que la substance transmet l'être par elle-même. De plus, la substance, dit-il, est et est pensée par elle-même, tandis que les autres ne le sont pas sans elle. Pour ces raisons, elle serait apparenté à ce qui est par soi et qui est délié de toute relation $(121,20-29)$.

Archytas attribue la première place à l'ov̉oí $\alpha$, parce qu'elle possède le statut de condition de possibilité des autres genres. Loin de la limiter à l'autosubsistance dans l'être, il la lui prête également dans la pensée. À la différence des autres catégories, il affirme qu'elle peut exister et être pensée par elle-même. Étant ce qu'il y a de plus proche de l'être par soi et exempt de relation, elle constitue le seul genre qui soit pensable par soi. Dans sa déduction, Archytas irait de ce qui subsiste le plus par soi vers ce qui est le plus en relation. Ensuite, une fois posée la catégorie de la relation, il irait de la forme de relation la plus pure à une forme non naturelle et acquise: à l'intérieur des relations, il partirait de ce qui est en relation par soi vers ce dont le caractère relatif provient d'un autre (cf. 334, 15-20). Bref, il respec-

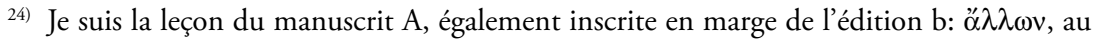
lieu de celle éditée par Kalbfleisch: ő $\lambda \omega v$ (JLKv). Parler subitement des touts dans ce passage n'a en effet aucun sens.
} 
terait le privilège de la simplicité, dans un esprit qui saccorde avec les attentes d'un commentateur néoplatonicien. De ce fait, il ne se livre pas à une enquête phénoménologique fondée sur l'expérience ordinaire, mais il étudie le concept de substance tel qu'en lui-même.

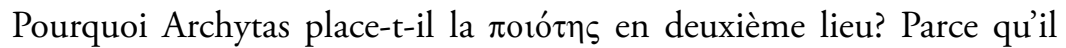
lui reconnaît une plus grande parenté avec l'ov̉ $\sigma i ́ \alpha$. Il distingue en effet à l'intérieur de cette dernière entre substance sensible et substance intelligible—qui reçoit un statut supérieur et retient l'attention d'Archytas. Or la quantité, en tant que grandeur et mesure, implique la corporéité. Par conséquent, elle concerne la seule substance sensible et ne se prédique pas de la substance intelligible. En revanche, la qualité se révèle un trait commun aux deux types de substances. Elle possède dès lors davantage d'universalité et mérite de précéder la quantité.

Archytas divise aussi la substance sensible en ov̉oía formelle, matérielle et composée. Au sein de cette tripartition, il attribue à la première (ov̉ $\sigma i ́ \alpha$

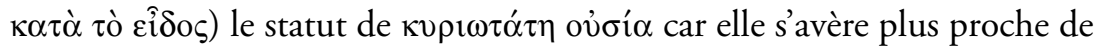
la substance intelligible. De plus, "la qualité est une certaine forme (عî̉os) et configuration ( $\mu$ óp $\varphi \omega \mu \alpha)$ appliquée à la substance ${ }^{25}$." En effet, eu égard à son incorporéité et à sa dimension révélatrice de l'eî̉os, le $\pi$ otóv constitue une sorte de forme à l'intérieur de la substance. En outre, étant sans partie, inétendue et divisible sans se morceler à travers les corps, la qualité s'avère plus proche des principes incorporels que la quantité, qui est étendue, divisible et réductible en parties. Dès lors, étant ce qui possède le plus d'affinités avec l'zî̉os, le $\pi$ otóv reçoit logiquement la deuxième place, juste après l'ở $\sigma i ́ \alpha$.

La qualité exprime mieux les substances, car c'est à leurs caractères et à leurs propriétés, à leur forme et à leur qualité, que nous les reconnaissons. Si nous supprimions la qualité, les caractères et les propriétés, tant intelligibles que sensibles, disparaîtraient, tandis que si la quantité venait à manquer, seuls le sensible et le composé en seraient affectés. Pour cette troisième raison, la qualité s'avère également plus générale et plus apparentée à la substance intelligible que la quantité. Elle mérite donc de recevoir la deuxième place $(121,29-122,4)$. Simplicius ajoute cette ultime

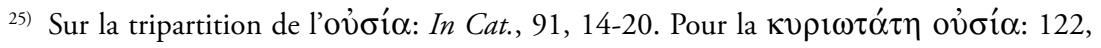
7-8; 206, 17-21. 
précision: la qualité ne peut jamais préexister à l'ov̉oí $\alpha$, car le $\pi$ oıóv $\tau \imath$ ne peut exister sans $\tau i ́$ ̌́ $\sigma \tau \imath$, la qualité requiert l'essence $(206,9-10)$.

Quant à Aristote, il n'adresse pas la question dans les mêmes termes qu'Archytas. D'une part, il reconnaît que l'ov̉oía n'est pas dite d'un sujet ni dans un sujet. Au lieu de s'interroger sur l'être et la pensée, il conclut sur le plan de l'énoncé. D’autre part, il privilégie, en lieu et place de la substance intelligible, la substance composée, car elle se révèle plus compréhensible à des débutants et plus accessible à nos habitudes linguistiques. Il s'en dégage qu'Archytas et Aristote prennent tous deux comme point de départ l'ov̉oí $\alpha$, mais qu'ils l'entendent en deux sens différents. Il n'y a dès lors rien d'étonnant à ce que chacun produise un ordre propre, en accord avec son postulat initial $(122,29-30)$. Archytas se montre $\dot{\omega} \varsigma$

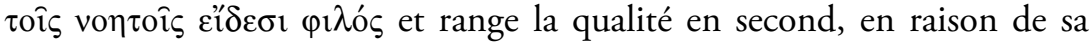

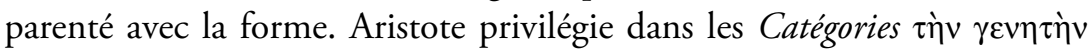

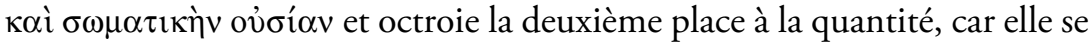
révèle plus corporéiforme $(157,26-32 ; 334,20-22)$. Cette variation du regard incite à nuancer la détermination du $\sigma \kappa о \pi$ ós: Archytas adopte le point de vue des formes intelligibles, se situant au-delà du langage ordinaire et du monde qu'il sert à décrire, tandis qu'Aristote écrit un traité de logique centré sur la substance sensible en devenir.

En revanche, ils s'entendent pour postposer les relatifs à la quantité. La relation succède à la quantité pour une double raison (158, 9-21). Naturelle, car la quantité se divise en quantité per se et en quantité pro alio; par conséquent, la relation doit être contiguë à la quantité. Elle ne peut néanmoins pas la précéder, étant donné que la grandeur ou le nombre qui fixent la valeur d'un objet quantifié (la quantité per se) préexistent aux termes comparatifs (la quantité pro alio). La quantité absolue existe dès lors antérieurement à la toute première forme de relation. De la même façon, l'excès et le défaut, qui sont connaturels à la quantité, impliquent l'existence de proportions, autrement dit de relations. Or, si la quantité appartient aux genres qui subsistent par soi, elle précède ceux qui manifestent une forme de relation. Pédagogique, car plusieurs termes qui appartiennent en apparence à la quantité s'avèrent relever de la relation (grand, petit, peu, beaucoup, etc.); par conséquent, il convient d'introduire la relation aussitôt après la quantité, en raison de cette connivence ${ }^{26}$. Sur ce point donc, les

26) En 158, 27-159, 8, Simplicius rapporte une justification alternative de l'ordre d'Aristote, 
ambitions des deux auteurs se rejoignent. Pourtant, malgré leur accord, Archytas et Aristote s'opposent, pour les raisons mentionnées, sur la place à attribuer à la qualité par rapport à la paire formée par la quantité et la relation: Archytas la range avant, Aristote après.

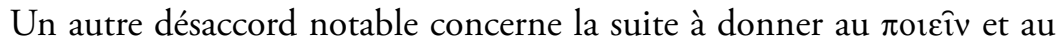

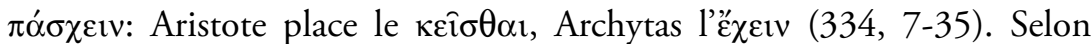
Simplicius, le premier suivrait un raisonnement finaliste et s'enquerrait de la position comme résultat de l'agir et du pâtir. Archytas par contre respecterait une logique qui s'accorde avec la doctrine néoplatonicienne et repose sur un triple privilège: celui de la relation la plus authentique (le

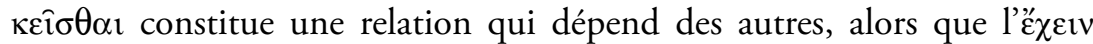
implique une relation de soi aux autres), celui de l'opération sur la pas-

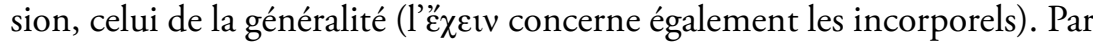
ailleurs, sur le plan de l'observation physique qui est celui d'Aristote, la position semble devoir précéder la possession. La première relation de tout corps est en effet celle qui se crée à l'égard de ce qui accueille sa position. Simplicius maintient donc qu'Aristote adopte une ligne de conduite phénoménologique centrée sur l'ov̉oía corporelle et composée, tandis qu'Archytas privilégie l'analyse logique et l'étude des genres purs posés dans les réalités les plus principielles. Car en identifiant Dieu à l'agir pur, par opposition au pâtir pur-la matière—, il retrouve les deux formes

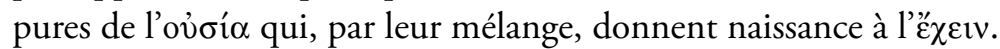

Simplicius insiste sur la nécessité de conférer un ordre aux catégories et révèle deux logiques possibles, à partir de l'opposition entre Archytas et Aristote. Toutefois, il lui arrive parfois de constater l'égale prétention de plusieurs genres à occuper une même place ( $\alpha \dot{v} \tau i \pi \lambda \varepsilon o v \varepsilon ́ \kappa \tau \eta \sigma ı \varsigma)$ et l'indif-

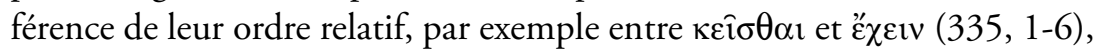

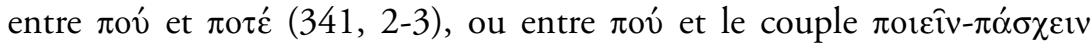
$(340,30-33)^{27}$. Il note des hésitations d'Archytas qui, sans rompre l'ordre

celle de Porphyre: la qualité résulterait d'une combinaison de quantité et de relation. Il juge l'argument facile d'accès et ingénieux. Il admet aussi qu'il s'accorde avec Empédocle (pour qui le monde naît de la combinaison des éléments) et Platon (chez qui les Formes sont faites de rapports proportionnels). Loin d'être absurde au sujet des composés, il ne convient cependant pas au raisonnement causal, qui renvoie nécessairement la pluralité à l'unité d'un principe.

27) Simplicius démarque ce dernier cas des autres, dans la mesure où il reçoit une justifica-

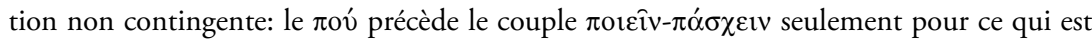


général, ouvrent à une alternative et, par là, justifient la voie adoptée par Aristote. Aussi, conclut-il, trancher entre $\pi$ ov́ et $\pi$ o $\tau \dot{\varepsilon}$ relève plutôt d'un usage des exégètes (décalque de l'ordre respecté par Aristote dans l'énumération) que d'une raison interne (341, 2-3; 342, 19-20). Loin de ruiner la thèse de la déduction nécessaire des genres, l'ambivalence des catégories, jointe à la souplesse inopinée du commentateur, sert de prétexte à une justification doctrinale. Elle l'autorise en effet à démontrer que toute réalité possède simultanément la double situation spatiale et temporelle, aucune ne pouvant se produire sans l'autre. Пov́ et $\pi$ o $\tau \dot{\varepsilon}$ apparaissent alors comme des genres jumeaux qui contribuent de façon égale à la génération et au mouvement, et qui, dès lors, méritent d'être classés ensemble (tout

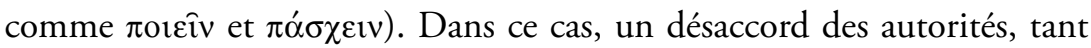
entre elles que vis-à-vis d'elles-mêmes, fournit la preuve de la nécessité d'un phénomène physique.

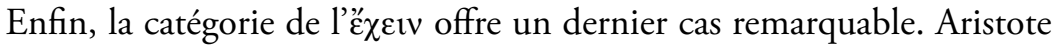
la cite en dernier lieu de son bref exposé sur les catégories finales, rompant avec l'ordre de la liste initiale. Archytas la range quant à lui à la suite immédiate du $\pi \alpha ́ \sigma \chi \varepsilon ı v$. Simplicius explique ce désaccord par une diffé-

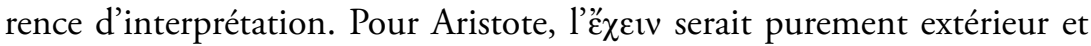
ne procèderait pas de soi-même de façon principielle—d'où sa place après

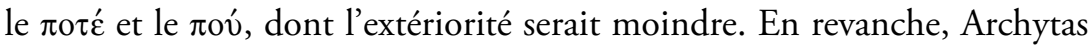
maintiendrait le lien homonymique entre la $\sigma \chi \varepsilon \dot{\sigma} \sigma \varsigma$ relationnelle et la

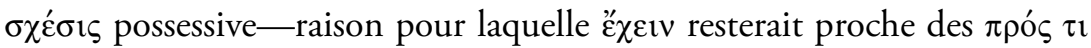
(365, 7-14). Simplicius introduit néanmoins une réserve (en recourant au " $\sigma \omega \varsigma$ indicateur):

Peut-être l'indique-t-il pour la raison que, bien que les genres possèdent un ordre, qu'Archytas fournit dans son exposé sur le sujet, les derniers se trouvent, en un sens, indifférents à l'égard de l'ordre, en raison de l'existence principielle et générique de tous (365, 21-24).

Cette invocation de l'indifférence pour expliquer le désaccord et les variations jette un doute manifeste sur le principe, pourtant si cher aux yeux

des substances corporelles, car la situation locale précède nécessairement l'action et la pas-

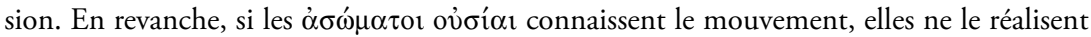
pas en un lieu. Cette explication étant posée par Simplicius, nous devons signaler qu'elle va à l'encontre de la citation qui sert à la corroborer. 
de notre exégète, d'un ordre révélateur d'une déduction a priori des catégories. Simplicius s'autorise à prendre la parole dans un contexte délicat, puisqu'il affaiblit l'autorité de ses prédécesseurs, Jamblique et Porphyre, très attentifs aux considérations taxinomiques.

Selon Simplicius, l'ordre dévoile l'importance et la signification des genres. À ses yeux, Archytas avait déjà pensé la nécessité d'une déduction des catégories et d'une organisation précise. Dans ces conditions, les variations entre Aristote et lui révéleraient des perspectives différentes, respectées dans le cas des premières catégories: empirico-phénoménologique, centrée sur la substance sensible et composée pour Aristote; noético-logique

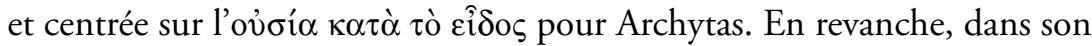
exégèse des dernières catégories, Simplicius rencontre plusieurs hésitations et se voit contraint de souligner l'indifférence de ces genres à l'égard de leur place respective. Il nuance la dimension déductive et fait preuve tantôt d'innovation tantôt de souplesse dans son activité de commentateur, vis-àvis de préceptes dont il notait pourtant la prégnance pour la saisie de l'ensemble.

\section{Un double inutile?}

Simplicius le montre: Aristote et Archytas n'écrivent pas le même traité. Le premier rédige une œuvre logique, destinée à des débutants en philosophie, afin d'exposer les mots simples qui signifient les êtres simples. Le second, en bon pythagoricien, révèle une vérité supérieure, qui manifeste un plus haut degré de pureté et d'unité, relative à un état antérieur de la pensée. Pourtant, malgré cette différence de points de vue, les deux auteurs s'accordent sur l'existence de dix genres et sur le sens à leur donner. En apparence du moins.

Bien que son premier effort soit de les estomper en vue de composer une image lisse et nette, Simplicius peint de l'harmonie entre Aristote et Archytas un tableau entaché de bigarrures. À côté des caractéristiques communes qu'il leur reconnait, il est contraint de constater plusieurs

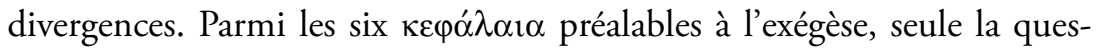
tion de l'authenticité reçoit une réponse dénuée d'ambiguïté. Quant au thème, à la place dans l'ordre de lecture, au découpage ou au titre, Simplicius doit ajuster le tir en fonction de développements successifs qui perturbent les hypothèses initiales, pourtant censées fournir une orientation rectiligne. 
Les deux traités résistent aux efforts de mise en parallèle systématique. Or, au lieu d'invoquer invariablement des différences entre les lecteurs visés (le lecteur pourrait s'attendre à ce qu’il généralise le principe de l'opposition entre publics cibles), Simplicius recourt parfois à des explications qui le mènent à contredire des postulats antérieurs. Devant l'incompatibilité de certains principes méthodologiques, il se voit conduit à composer, quitte à se distancier ou à relativiser certains apports de ses autorités-les commentaires de Porphyre et de Jamblique.

Dès lors, au vu des divergences et des artifices déployés pour restaurer la $\sigma u \mu \varphi \omega v i ́ \alpha$, pourquoi Simplicius persiste-t-il à soutenir la correspondance entre Archytas et Aristote? Ses efforts résultent de la tâche inhérente au travail de l'exégète tel qu'il le conçoit. À la différence des commentaires modernes, qui visent à une interprétation scientifiquement irréprochable d'un texte (sans tenir compte des ambitions moins nobles qui se cachent derrière toute publication scientifique aujourd'hui), le commentaire tardoantique tel que Simplicius le rédige poursuit en outre une finalité psychagogique $^{28}$. Il se révèle un lieu privilégié pour l'élévation de l'âme, par la mise en contact qu'il rend possible avec des connaissances qui excèdent notre monde. Dans ces conditions, en même temps qu'il découvre les principes élémentaires de la logique, le disciple rencontre, à la faveur d'excursus, les fondements de l'ontologie pythagoricienne-passant de ce fait des termes simples aux réalités elles-mêmes qu'ils servent à désigner. Bref, intégrer Archytas à l'explication des Catégories d'Aristote, c'est mener l'âme du lecteur vers un degré plus élevé du réel, par la confrontation avec des doctrines touchant à un domaine plus élevé du savoir.

\section{Une toile de fond}

Si, dans l'esprit de Simplicius, Archytas sert de modèle à Aristote, il n'y a rien d'étonnant à ce qu'il apparaisse de façon récurrente au sein du Commentaire sur les Catégories. Dans ces circonstances, analyser l'usage qui y est fait d'Archytas constitue un moyen d'améliorer notre compréhension de la méthodologie du commentateur, mais aussi d'approfondir nos connaissances de son interprétation des Catégories et, au travers, de celle

28) Pour une autre illustration de ce procédé, lire notamment la contribution de Brisson (2000) sur Proclus. 
de Jamblique. L'analyse des passages concernés révèle que Simplicius invoque Archytas pour quatre raisons principales: fournir une autorité, gagner en précision, relever une opposition, concilier.

\section{Archytas: la plus haute autorité}

Par rapport à Aristote, Archytas jouit du double privilège de l'ancienneté et de l'appartenance au pythagorisme. Il représente une autorité prestigieuse à utiliser à bon escient, qu'il s'agisse de confirmer un propos d'Aristote ou de lever une aporie à son encontre. Dès lors, examiner l'ascendant d'Archytas sur Simplicius et les circonstances de son invocation indiquera si notre commentateur recourt à l'argument d'autorité, selon quelle limite et à quelle fin.

D’après Simplicius, Aristote a suivi dans les Catégories un modèle pythagoricien provenant de deux sources. La deuxième partie-l'exposé des dix catégories - transcrirait le traité Sur les notions universelles d'Archytas, alors

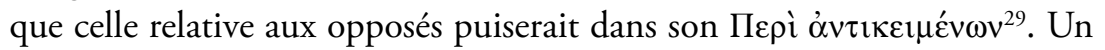
désaccord apparaît d'emblée: Archytas n'a rien exposé sur les noms d'analogue au début des Catégories. L’omission, que Simplicius juge volontaire, ne manque pourtant pas de susciter sa réflexion (40, 5-13): les Pythagoriciens posent une correspondance naturelle entre nom et réalité ( $\varphi v ́ \sigma \varepsilon \imath$ ). Sur ce point, ils s'opposent à Aristote, qui attribue aux noms une origine conventionnelle $(\theta \varepsilon \dot{\sigma \varepsilon l})$. Cet écart a retenu l'attention de ses prédécesseurs, comme le constate Simplicius:

Les Pythagoriciens ont élevé à dix le nombre de simples, comme Archytas (que Platon a fréquenté) l'a enseigné dans son livre Sur les notions universelles, suivi par Aristote jusque dans l'emploi des noms. Selon certains [i.e. Jamblique], il ne s'en écarte que dans cette seule mesure: il n'ajoute pas à ce

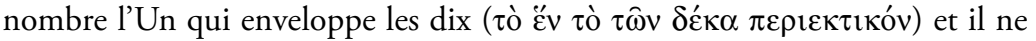

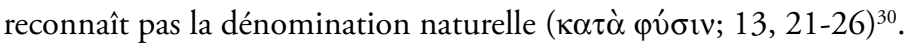

29) Cf. In Cat., 2, 15-25; 13, 21-26; et In Cat., 382, 8-10; 409, 7-10; 411, 29-30; 416, 22-27; 417, 15-17.

30) Simplicius néglige le premier point. Signifie-t-il qu'il existerait un Un enveloppant les dix termes universels? Cette affirmation poserait énormément de difficultés, dans la mesure où les dix genres sont censés être généralissimes, par conséquent privés de la possibilité d'une définition. Or, si dans la perspective du néoplatonisme tardif chaque genre doit être considéré 
Pour résoudre la difficulté relative à la dénomination naturelle, Simplicius invoque une doctrine intermédiaire. D'après la première institution du langage, les noms possèdent une origine conventionnelle. Or cette dénomination fut le fait des premiers onomatothètes, qui connaissaient la nature des êtres qu'ils souhaitaient désigner au moyen de ces noms. Il est donc correct de parler d'une position conventionnelle du langage mais en l'assortissant d'une connaissance de la nature des réalités (20, 4-7). Le désaccord entre Aristote et les Pythagoriciens est désormais levé au moyen de cette doctrine originale, qui associe nature et convention. Celle-ci soulève néanmoins un problème, dans la mesure où, selon Simplicius, aristotélisme et pythagorisme reposent sur des idées distinctes de la nature: si les Catégories l'identifient au monde phénoménal, les Pythagoriciens—suivis en cela par Platon-se réfèrent à des réalités transcendantes et examinent les réalités naturelles en tant qu'elles participent à ce qui dépasse la nature empirique $(6,27-30)$. Il resterait encore à expliquer comment s'articulent ces deux conceptions de la nature-nature transcendante et nature empirique.

La solution suscite par ailleurs une remarque méthodologique: Simplicius rapporte le jugement de ses prédécesseurs (sans doute Jamblique) mais s'en démarque sur deux points. D'une part, au fil du commentaire, il signalera un bien plus grand nombre de désaccords. D'autre part, il résout ce qui était apparu à Jamblique comme un point insurmontable de divergence entre Archytas et Aristote, restaurant du même coup leur harmonie. Dès lors, s'il mentionne davantage d'écarts, il le fait en vue d'accroître la concorde entre ces autorités, car plus leurs doctrines feront entendre de dissonances et plus leur dissipation manifestera l'accord profond qui les anime.

Modèle structurel, Archytas sert aussi d'autorité pour le choix, le nom et le nombre de genres. Si la division aristotélicienne en dix catégories peut paraître arbitraire, elle bénéficie à ce titre d'un appui non négligeable, puisqu'elle puise à la source du pythagorisme.

Procédant sur le mode pythagoricien, Archytas ramène la raison du nombre dix aux principes universels des êtres. Il affirme en effet que tout art et toute

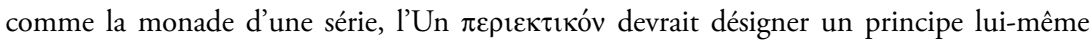
cause de la forme d'unité que tous les genres ont en commun dans leur définition. 
science est une réalité ordonnée et déterminée. Or une telle réalité est définie dans un nombre; de plus, la totalité du nombre est la décade; il est par conséquent vraisemblable que toutes les choses soient divisées en dix, que les espèces en leur totalité et les nombres essentiels soient dix; en outre que les extrémités du corps aient dix parties; et que les éléments du discours dans sa totalité soient dix $(68,22-28)$.

Attribuer à Aristote la division du réel en dix genres principiels et en dix nombres essentiels, c'est s'autoriser à l'intégrer à la tradition pythagoricienne, autrement dit à le resituer dans un contexte plus large: s'il n'a pas explicitement évoqué la relation entre l'Un et les dix genres, le principe de la décade confère à lui seul une source d'unité. En dépit des apories soulevées dans la Métaphysique à l'encontre des principes pythagoriciens (en particulier dans les livres $\mathrm{M}$ et $\mathrm{N}$ ), Aristote ne s'exclurait pas de cette tradition de pensée, qui part de Pythagore et passe par Platon. En soulignant la filiation entre la doctrine aristotélicienne et Archytas, Simplicius consacre la continuité de la pensée païenne, lieu de manifestation de l'unité métaphysique de la réalité. Dans ces conditions, toute objection adressée aux catégories d'Aristote se révélera au premier chef une attaque contre le système des principes pythagoriciens $(67,22-25)$.

Cette mise en garde ne satisfait pourtant pas pleinement Simplicius qui, suivant certainement Jamblique, ajoute aussitôt une autre raison d'avaliser l'existence de dix genres. Il suffit en effet pour s'en convaincre de

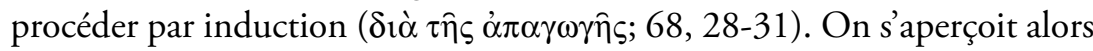
que toute réalité, quelle qu'elle soit, peut être reconduite à l'un des genres mentionnés par Aristote. Si l'autorité pythagoricienne doit convaincre à elle seule, il n'est pas inutile d'ajouter une preuve empirique pour réduire les sceptiques au silence $(7,33-8,8)^{31}$.

L'utilisation d'Archytas autorise également Simplicius à tirer des conclusions doctrinales. En juxtaposant les textes, Simplicius n'entend pas

\footnotetext{
31) Il existe plusieurs cas similaires. Archytas avait déjà affirmé que l'ov̉oía était un

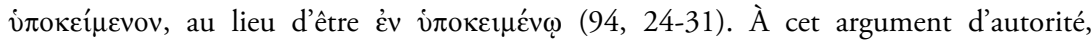
Simplicius ajoute une raison. Puisque la matière constitue le premier sujet, elle ne peut pas être elle-même dans un sujet; de même, la forme ne se trouve pas dans la matière comme dans un sujet. Par conséquent, le composé des deux se révèle un sujet, et non être dans un sujet. Ou, plus loin, Archytas avait déjà affirmé qu'être positionné signifiait être positionné dans, bien que le lieu lui-même ne soit pas en un lieu: étant lui-même limite des corps, il ne requiert pas d'autre limite qui l'entoure $(337,32-34)$.
} 
seulement corroborer un argument d'Aristote. Par l'accord manifeste entre les deux auteurs, il désire plutôt souligner la vérité fondamentale et commune qui inspire leurs écrits. Par exemple, à l'instar d'Aristote, Archytas

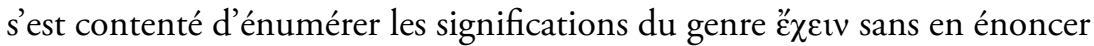
les différences spécifiques. Cette communauté de vue, qui pourrait passer pour une imprécision, apparaît à Simplicius comme un élément essentiel de la théorie de ce genre, car elle prouve l'impossibilité de se montrer plus complet (367, 3-13). En d'autres termes, Aristote et Archytas n'énoncent pas seulement des doctrines explicites; joints l'un à l'autre, ils en dispensent également entre les lignes.

Enfin, Archytas joue le rôle de repoussoir à l'égard d'apories soulevées contre Aristote. Sans dispenser le commentateur d'une raison, il offre un surcroît d'argument censé conférer une autorité aux Catégories. La seule mention de son nom augmente le crédit et avalise le propos d'Aristote contre ses détracteurs. Simplicius joue ainsi à plusieurs reprises Archytas contre Plotin. Dans les Genres de l'Etre (VI, 1, 24), ce dernier soutient que

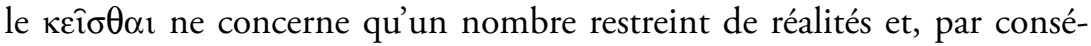
quent, ne mérite pas de faire l'objet d'un genre propre. Se réclamant d'Archytas, Simplicius lui objecte qu'à partir du moment où il appartient à tous les corps supralunaires, pour lesquels il faut distinguer entre confi-

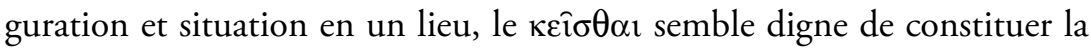
catégorie qui rend compte de la seconde ${ }^{32}$.

Simplicius joue sur plusieurs tableaux de l'autorité. En un premier sens, l'autorité renvoie à ses prédécesseurs, commentateurs des Catégories. En bon exégète, Simplicius se met en quête de la raison d'une difficulté relevée avant lui, plutôt que de polémiquer en surface. Il n'hésite pas à se détacher de sommités telles que Plotin ou Jamblique, qui se révèlent des autorités à vérifier par l'épreuve du texte. En un second sens, il recourt à l'autorité à titre d'argument. Archytas remplit ce rôle à plusieurs reprises dans l'examen des Catégories, survenant contre divers types d'objection adressées à Aristote, qu'il s'agisse d'un manque de précision, d'un reproche

32) In Cat., 338, 21-33. Cf. 76, 17-23, où Simplicius signale une objection de Plotin (VI, $1,2)$ et de Nicostrate relative à la possibilité de former un genre unique de la substance, au vu de la division entre substance sensible et substance intelligible. Simplicius cite Archytas pour rappeler que l'objet actuel, tant des Catégories que Des notions universelles, est la substance naturelle et discursive. Cf. 317, 12-22, où il cite la division archytéenne des $\pi$ oîعıv et $\pi$ ó $\sigma \chi \varepsilon ı v$ contre les arguments de Plotin. 
d'arbitrarité d'une doctrine ou d'une accusation d'égarement par rapport à la réalité. Là où le Pythagoricien avait déjà tenu un propos similaire, Aristote ne peut aucunement s'être fourvoyé. Dans ces conditions, le déploiement de l'explication d'Aristote apparaît en même temps comme la justification du propos d'Archytas. Cela n'empêche pas le commentateur de produire à l'occasion une preuve supplémentaire, fondée sur l'induction ou sur la raison.

\section{Un gain en précision}

Aux yeux de Simplicius, Archytas constitue la source sur la doctrine des genres premiers à laquelle Aristote a abondamment puisé. Aussi le commentateur n'hésite-t-il pas à s'y plonger à nouveau, dans le but de combler d'éventuelles lacunes. Là où Aristote manque de précision, le Pythagoricien offre un complément utile d'information.

Parlant en son nom propre, Simplicius accepte la définition archytéenne de la substance, parce qu'il la juge plus complète que celle d'Aristote. En effet, le propre de la substance ne se limite pas à être dans un substrat, comme l'affirment les Catégories, mais d'être par soi, ainsi que le dit $\operatorname{Archytas}^{33}$. Alors qu'Aristote se contente d'une détermination négative et énonce le non-propre de l'ov̉oía-ne pas être dans un substrat-, Archytas a déjà apporté l'enseignement qui atteint la substance dans sa positivité $(116,11-17)$. Plus complet, il l'est également quand son texte, apparemment similaire en tous points à celui d'Aristote, contient l'élément qui lève une ambiguïté manifeste.

Dans ce passage, [Archytas] énonce la même propriété de la substance et, au

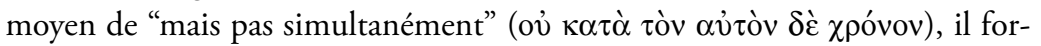
mule la raison du changement successif des contraires. De plus, il résout les apories tirées des attributs connaturels ${ }^{34}$, des mobiles éternels ${ }^{35}$ et des êtres

\footnotetext{
33) In Cat., 96, 24-30: preuve que Simplicius l'admet en première personne, il commence

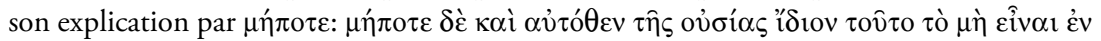

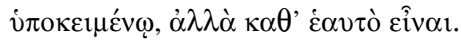

34) Si le chaud constitue la propriété essentielle du feu, ce dernier ne le reçoit pas comme une propriété extérieure. Par conséquent, le chaud n'est pas un propriété acquise pour le feu, comme c'est le cas pour l'eau (114, 31-36).

35) Étant toujours en mouvement, le Soleil ne peut s'arrêter. Par conséquent, bien qu’il
} 
éternels ${ }^{36}$ : tous ceux-ci ne surviennent pas selon le temps. Et il résout les disputes provenant des genres en ajoutant "demeurant" $\left(\delta ı \mu \mu^{\prime} v o \imath \sigma \alpha v\right)$, puisque ceux-ci ne demeurent pas $^{37}$ : ce n'est pas la couleur dans le blanc que reçoit le noir, mais il se retire avec le blanc et le même ne demeure plus (116, 17-24).

Traitant de la possibilité pour la substance d'admettre les contraires,

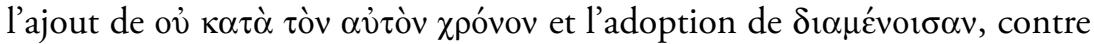
óv chez Aristote (4 a 10-11), résolvent la double aporie relative au fait d'être une et identique tout en acceptant les contraires. D'un côté, ce qui échoit à l'éternité, tant de l'être que du mouvement, et qui refuse de toute éternité un état contraire ne relève pas, par essence, de la temporalité: Archytas exclut de la définition les substances éternelles et, de ce fait, évite l'objection selon laquelle certaines substances ne pourraient pas recevoir les contraires. D'autre part, ce qui est se refuse au changement requis par la nécessaire succession des contraires au sein de la substance: bien qu'elles soient, les substances secondes, genres et espèces, ne sont pas des réalités susceptibles de demeurer. Comparée à celle d'Aristote et en dépit de la similitude apparente, la leçon d'Archytas sur la substance s'avère, à maints égards, plus précise et moins équivoque $e^{38}$.

À l'inverse, Simplicius estime que le gain en précision provient parfois d'un silence délibéré d'Archytas qui, ce faisant, évite une confusion suscitée par Aristote (6 b 15-19). Par exemple, s'abstenir de mentionner les contraires à propos des relatifs rappelle que ces derniers ne sont contraires que $\kappa \alpha \tau \grave{\alpha} \sigma v \mu \beta \varepsilon \beta \eta \kappa o ́ \varsigma$, puisque la contrariété résulte de leur présence à l'intérieur de substrats eux-mêmes contraires: la vertu et le vice ne sont pas contraires parce qu'ils sont relatifs, mais en tant qu'ils renvoient à des qualités contraires. Archytas se serait gardé d'une telle précision afin d'éviter aux lecteurs une confusion entre la relation elle-même et les sujets de la

soit une substance (qui a la particularité d'être toujours mue), il n'admet pas le contraire-à savoir le repos $(114,23-31)$.

36) Les substances intelligibles n'admettent pas les contraires (114, 21-23).

37) En tant que substances secondes, les genres et les espèces sont susceptibles des contraires: l'homme peut être craintif et hardi, le mouvement rapide et lent, etc. En ce sens, la substance admettrait les contraires $(114,5-20)$.

38) In Cat., 278, 30-33: Archytas parle avec plus de précision, lorsqu'il range au sein de la qualité non seulement la contrariété, mais également la privation. Simplicius loue constamment son acribie $(290,11-12 ; 438,31-32)$. 
relation: la relation est un genre commun qui unit des extrêmes, mais elle est elle-même différente de ces extrêmes dans lesquels s'observe la contrariété (178, 16-179, 4). En bref, Simplicius emploie l'argument a silentio pour relever la supériorité scientifique d'Archytas ${ }^{39}$.

Il lui arrive également de l'utiliser pour procéder à une forme d'harmonisation dans le silence. Archytas introduit en effet une médiété ( $\mu \varepsilon \sigma o ́ \tau \eta \varsigma)$ entre les relatifs $(384,7-13)$ : l'homme libre se situerait à mi-chemin du couple de relatifs que forment le maitre et l'esclave ${ }^{40}$. Aristote ne dit rien de tel ou, plutôt, n'en parle pas du tout. Aussi, Simplicius prétend que si ce dernier ne l'avait pas admise, il aurait formulé une définition plus complète des relatifs, qui aurait évité toute ambiguïté: devant une doctrine dont Aristote ne fait nulle mention mais qu'il ne rejette pas explicitement, Simplicius voit dans le silence une simple omission qui l'autorise à rétablir l'harmonie. Par conséquent, le silence lui sert non seulement à manifester la supériorité du Pythagoricien, mais aussi à justifier par provision la résolution d'éventuels désaccords.

Toutefois, c'est surtout le silence d'Aristote qui laisse un monde à occuper et oblige l'exégète à combler les lacunes. L'absence d'analyse des six dernières catégories le confronte en effet à une double difficulté. D'une part, il lui échoit d'expliquer pourquoi le Stagirite s'est abstenu d'en traiter. D'autre part, l'ambition didactique le contraint à des explications complètes, pour lesquelles il lui incombe de se doter d'un support autoritatif-le commentaire ne devant en aucun cas se transformer en production d'idées nouvelles. Concernant le premier problème, Simplicius invoque une raison simple: l'évidence. Ce débutant qu'est le lecteur d'Aristote se satisfait de quelques remarques rapides, car le sens du $\pi$ ov́ ou du $\pi$ o $\tilde{\varepsilon}$, par exemple, saute aux yeux. Quant au second problème, il justifie la

39) La situation inverse-un silence d'Archytas explicité par Aristote-, a été évoquée à propos du бколós. Dans ce cas, Simplicius oppose le point de vue unitaire d'Archytas à la multiplicité d'Aristote: par exemple, tandis que le premier se limite à l'identité de l'espèce et ne se soucie pas de diviser $\tau$ ò $\dot{\varepsilon} v \ddot{\varepsilon} \xi \varepsilon 1$, le second apporte des développements sur la $\delta i \alpha ́ \theta \varepsilon \sigma ı \varsigma$, sans pour autant s'écarter de l'hypothèse commune (cf. 240, 20-27; 272, 2-6).

40) L'idée de médiété se trouve dans un fragment authentique d'Archytas. En musique, il distingue trois formes de moyenne ( $\left.\mu \varepsilon \varepsilon_{\sigma \alpha}\right)$ : arithmétique, géométrique et subcontraire. Or,

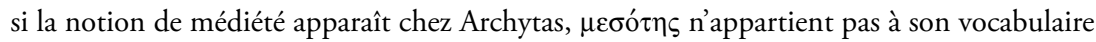
mais plutôt à celui de l'auteur qui le cite-Porphyre (B 2 = Porphyre, Commentaire sur les Harmoniques de Ptolémée, éd. Düring, 92-93). 
prégnance d'Archytas. Le Pythagoricien ne sert plus seulement de point de comparaison ou de texte parallèle. Il devient la source principale du commentaire, autour de laquelle s'organisent les analyses, les objections et leurs réponses. Pour les petites catégories (celles négligées par Aristote), il confere l'ordre de l'examen, ainsi que toutes les divisions, les descriptions, les propres et les différences ${ }^{41}$. Dès ce moment, c'est son texte qu'il s'agit d'expliquer, plutôt que celui, déficient, d'Aristote.

41) Comme l'exposé aristotélicien se raréfie à partir de l'agir et du pâtir, Archytas devient le moteur de l'exégèse de Simplicius. Ce dernier s'emploie à déplier le commentaire de Jamblique, fondé lui-même sur une explication de l'extrême brachylogie d'Archytas. Ce processus leur permet de rétablir les doctrines suivantes.

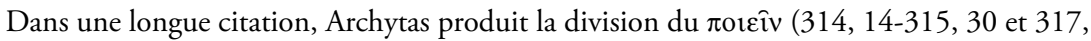
6-319, 15):

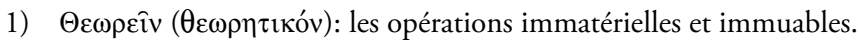

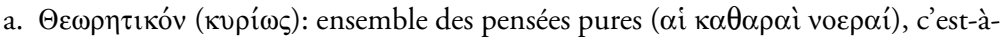

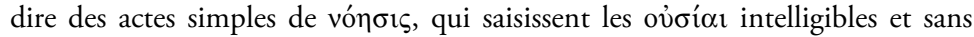
partie. Archytas n'en parle pas car, selon Simplicius, cette espèce n'est pas appropriée à une introduction.

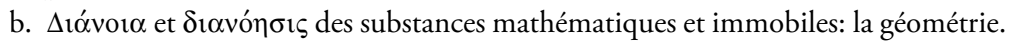
Elle fonctionne par intuition définie et composée.

c. Orwpí $\alpha$ des substances sensibles éternelles et toujours mues, qui est une perception accompagnée de raisonnement (oi $\mu \varepsilon \tau$ ' $\alpha i \sigma \theta \eta ́ \sigma \varepsilon \omega \varsigma \delta i \alpha \lambda o \gamma ı \sigma \mu o i ́)$ : l'astronomie.

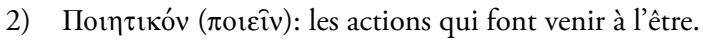

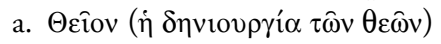

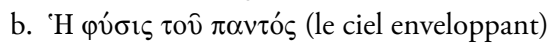

c. Les arts qui imitent la nature en inscrivant la forme dans le corps: le médecin qui produit la santé.

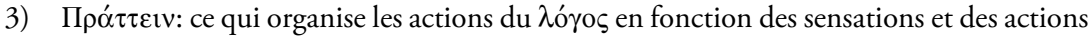
composées (choix, désir, opinion, etc.). Cette espèce relève de la capacité à former des opinions. Elle est en relation avec les sensibles et admet la vertu et le vice. Selon cer-

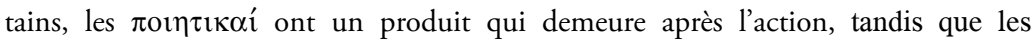

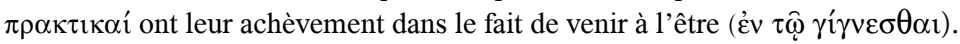

4) Avev̀ $\delta 1 \alpha v o i ́ \alpha \varsigma$ : les actions des animaux non rationnels (Simplicius renvoie aux traités biologiques).

Jamblique ajoute les actions des corps inanimés-les opérations des éléments ou les mouvements d'inclination. Simplicius juge qu'il s'agit d'une catégorie mixte, très étendue et qui concerne la classe la plus basse. Selon lui, Archytas n'en parle pas pour la raison qu'il ne s'occupe que des actions au sens propre, qui ont en elles-mêmes leur principe de mouvement, et qu'il rejette les actions mêlées de passion (319, 12-15).

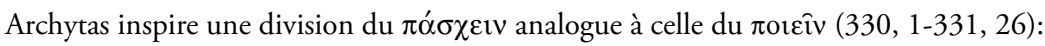


Archytas prouve la possibilité d'un traitement plus étendu que celui d'Aristote à propos des six dernières catégories. Leur opposition relève d'une différence de perspective. D'un côté, Aristote fournit un enseignement complet et précis sur ce que n'assument pas absolument les notions communes. Pour le reste, il juge leur évidence suffisante. De l'autre côté,

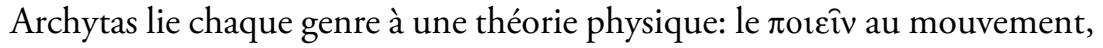
le $\pi$ ov́ au lieu, le $\pi$ o $\tau \varepsilon$ au temps. Or un traité de logique ne justifie pas de tels développements. C'est donc à juste titre qu'Aristote ne va pas au-delà de la connaissance manifeste relative à la signification. Quant à Archytas, il vise un autre objet et se montre plus complet concernant les réalités (300, 9-30).

À côté des informations de contenu, Archytas sert de garant à des précisions méthodologiques et conceptuelles. Il recommande ainsi d'appliquer à chaque catégorie un traitement propre et adapté "à la notion de sa propre nature" (

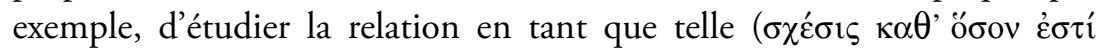
$\sigma \chi \varepsilon \dot{\varepsilon} \varsigma \varsigma)$, et non en tant que les relatifs possèderaient des traits communs avec la qualité. S'ils acceptent le plus et le moins-comme tout à l'heure la contrariété-, c'est uniquement par accident (179, 19-21). En outre, pour revenir à des considérations relatives au mode d'exposition, Simplicius

1) Пó $\theta$ os: ce qui suit la sensation et persiste à travers l'altération. Cette espèce inclut les

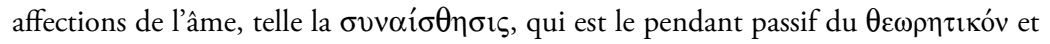
qui possède les mêmes degrés.

2) ПвлоvӨós: les altérations inconscientes et les passions insensibles qui surviennent dans la nature, entraînant un changement clair au terme du processus. Cette espèce s'oppose aux actions des inanimés.

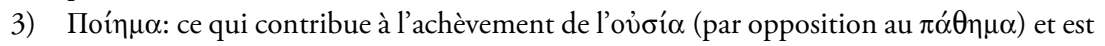

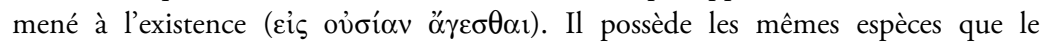
$\pi$ oin $\tau$ кóv.

4) П'́d $\theta \eta \mu \alpha$ : les affections qui ne s'achèvent pas dans un produit fini.

Plus brève, la division du кعiَ $\theta \alpha$ aboutit à ceci $(338,9-20)$ :

1) $\dot{\varepsilon} v \delta v \vee \alpha ́ \mu \varepsilon เ$

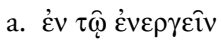

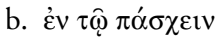

2) $\dot{\varepsilon} v \sigma \tau \alpha \dot{\alpha} \sigma \varepsilon 1$

Pour les autres catégories, In Cat., 351-357: sur le temps; 361-363: sur le lieu; 365, 25-366,

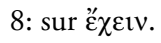


prête à Archytas une recommandation en vue d'un ordre plus logique. S’agissant de diviser la qualité,

Archytas semble placer l'espèce de la qualité relative à l'affection avant celle relative à l'être en puissance; peut-être est-ce plus logique, parce que ce qui est en acte doit précéder ce qui en puissance, quel qu'il soit $(259,17-20)$.

Archytas n'est donc pas seulement pourvoyeur de doctrines, mais également de la façon dont il convient de les examiner.

Dans la comparaison avec Aristote, Archytas l'emporte en précision, mais aussi en clarté et en concision. Si le premier se montre prolixe, le second préfère la brachylogie. Cette situation duelle laisse le champ libre au commentaire, car elle ouvre un espace pour l'explication. Dans ces conditions, Simplicius se livre à un double usage du silence. Tantôt il tire argument d'un silence d'Archytas pour justifier une doctrine obscurcie par l'abondance d'Aristote. Tantôt, au contraire, il recourt au texte d'Archytas pour pallier un silence d'Aristote, plus ou moins long. Une addition du Pythagoricien, si minime soit-elle, apparait alors comme un frein aux apories et aux objections adressées aux Catégories. Puisqu'il se destine à un public plus expérimenté, Archytas déploie des doctrines plus abouties, qui requièrent une plus grande concentration intellective. Sa contribution peut toutefois s'étendre bien au-delà des propos du Stagirite. Elle sert alors de patron à l'exégèse et fournit la doctrine nécessaire à un commentaire complet.

\section{L'écart par rapport à l'autorité}

Il serait bon d'ajouter quelques considérations sur la relation de Simplicius à Jamblique. Si, le plus souvent, il suit fidèlement son Commentaire et adopte sa lecture d'Archytas, il s'autorise de temps en temps un pas de côté. Nous avons rencontré quelques passages où Simplicius se risquait à

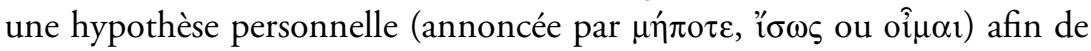
combler une lacune laissée par son modèle. Ailleurs, il ajoute, de façon explicite et par souci de complétude, de longues citations à son commentaire, preuve peut-être qu'il détenait un manuscrit d'Archytas (92, 27-93, $9 ; 407,15-409,12)$. Il mentionne également certaines situations où Jamblique a emprunté des distinctions conceptuelles proposées par Archytas ${ }^{42}$.

42) Cf. In Cat., 330, 1-14: énonçant les divisions qu'Archytas propose pour le $\pi \alpha ́ \sigma \chi \varepsilon ı v$, 
Autrement dit, par le biais de sa lecture d'Archytas, Simplicius nous fournit de précieuses informations sur l'état et le contenu du Commentaire perdu de Jamblique, notamment par les quelques objections qu'il adresse à son interprétation.

Simplicius reproche à Jamblique de s'éloigner du projet d'Archytas dans sa résolution d'une aporie soulevée contre lui. Alors que ce dernier établit que l'agir possède nécessairement la cause de son activité en lui-même, certains adversaires lui objectent qu'il existe des actes qui tireraient d'ailleurs la cause de leur activité, par exemple l'intelliger. Jamblique leur répond que la cause de l'intelliger ne peut pas entièrement provenir de l'être-intelligé, puisque celui-ci est en quelque sorte un pâtir par rapport à l'intelliger.

Cet argument est propre à la théorie relative à l'intellect. Cependant, il faut résoudre l'aporie conformément à ce qui est sous nos yeux, en disant, je pense, qu'Archytas a rendu compte des actions et des passions pures. Or, bien qu'il soit une sorte de pâtir, l'intelliger a néanmoins une activité éveillée de l'intérieur, tout comme le voir et, en un mot, le connaitre. Jamblique ne devait pas relever cette objection formulée contre le propos d'Archytas à partir de ces réalités mélangées (315, 1-6).

Simplicius ne conteste pas la solution de l'aporie en tant que telle. Il reproche à Jamblique de ne pas l'avoir résolue dans les termes d'Archytas. Pour Simplicius, l'exégète doit se maintenir dans les limites de l'œuvre qu'il commente, se servir des outils conceptuels qu'elle expose et répondre aux objections en veillant aux limites et aux objectifs qu'elle se fixe (cf. 319, 10-15). En d'autres termes, Jamblique aurait dû se contenter de nier l'objection, dans la mesure où elle manquait son but en formulant une critique hors de propos.

Enfin, de même que Simplicius joue parfois une autorité contre l'autre, il lui arrive aussi de réunir leurs propos pour opposer à Jamblique l'accord qu'il lit entre elles. À propos du lieu, il rapporte la voepò $\theta \varepsilon \omega p i ́ \alpha$ de Jamblique, qui se fonde sur la thèse d'Archytas ${ }^{43}$. D'après celle-ci, le lieu

Simplicius ajoute aussitôt qu'elles ont été reprises et développées par Jamblique, autrement dit bien intégrés à la doctrine scolaire.

43) In Cat., 361, 7-362, 16: la doctrine intellective de Jamblique peut être résumée par les points suivants: 
délimite les corps. Simplicius cite l'ı́ $\delta$ ıov du lieu tel qu'Archytas le définit et dont Jamblique s'inspire:

Le propre du lieu est que le reste est en lui, mais que lui n'est en rien. Car s'il est en un lieu, le lieu lui-même sera en retour dans un autre lieu, et cela arrivera jusqu'à l'infini. Par conséquent, il est nécessaire que le reste soit en un lieu, mais que le lieu ne soit en rien. Mais il est alors pour les êtres dans le même rapport que les limites par rapport aux limités, car le lieu de l'univers tout entier est la limite de tous les étants $(363,22-27)$.

Simplicius note que Jamblique étend ce propre à l'âme et à l'intellect. Il soutient que l'action de l'intellect, simple et unifiée, constitue la limite de l'univers. Au-delà, il existerait une ov̉oí $\alpha$ du lieu qui serait un dieu, qui

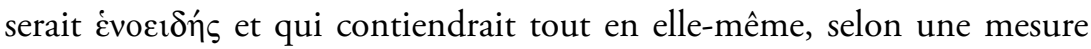

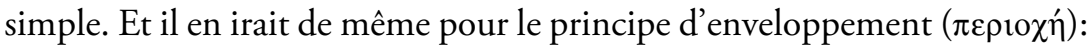
sa cause nous conduirait vers un lieu divin qui serait cause de lui-même et s'inclurait lui-même, subsisterait en lui-même et aurait une réalité qui ne serait plus séparable de ce qui est $(363,29-364,6)$. Mais Simplicius conteste cette interprétation:

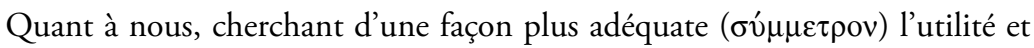
la nature du lieu au sens propre, et non dit par métaphore, nous affirmons que toutes les réalités inengendrées et sans partie, qui possèdent la substance et l'extension unifiée de l'être, sont tout entières en même temps en elles-mêmes et, pour cette raison, n'ont besoin ni de lieu ni de temps, mais là-bas suffit l'éternité, qui fait que tout reste dans l'un. De l'autre côté, les réalités engendrées et en parties, qui possèdent leur substance elle-même et l'extension de leur être, ont besoin d'un temps qui ne laisse pas être dispersée ni

- Le lieu est antérieur aux corps et les conditionne.

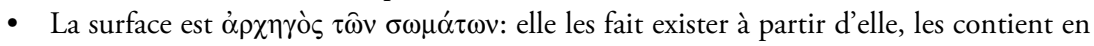
elle et les définit.

- Les corps ont leur être dans le lieu: ils sont contenus en lui et confinent leur extension à sa nature inétendue.

- Incorporel, le lieu est supérieur à ce qui est en lui: il est la limite commune, mais pas simple ( $\psi 1 \lambda$ òv $\pi \varepsilon ́ p \alpha \varsigma:$ comme les surfaces mathématiques sont les limites des corps mathématiques), qui décrit la limite physique des corps physiques. En bref, les limites doivent êtres adaptées à ce dont elles sont les limites. 
confondue l'extension de leur être, mais l'unisse et la connecte autant que possible dans l'ordre de l'antérieur et du postérieur (364, 7-15).

Simplicius se démarque de Jamblique sur deux points. Premièrement, il prétend tenir un propos plus adapté à l'objet actuel, qu'il soit celui d'Archytas ou d'Aristote. Deuxièmement, il met au jour une opposition qui avait échappé à Jamblique. D’une part, il existe des choses inengendrées et indivisibles, qui n'ont besoin ni du temps ni du lieu, mais qui possèdent leur être en elles-mêmes et se maintiennent dans l'unité grâce à l'éternité. D'autre part, les choses engendrées, qui possèdent leur être et la continuité de façon divisible, ont besoin du temps et du lieu. Du temps parce qu'il permet d'éviter la dispersion et la confusion de leur être, tout en les unifiant et en les liant dans un ordre, selon l'avant et l'après. Il empêche que des événements distincts et distants ne viennent à se mélanger, en maintenant leur succession. Il rend impossible que la guerre de Troie et l'instant présent ne se superposent subitement. Du lieu parce qu'il reçoit les parties des corps, les organise, les arrange et les définit, de sorte qu'elles ne se mélangent pas en se superposant. Grâce à lui, chacune occupe une place appropriée.

Simplicius distingue donc deux sortes de lieu et de temps:

1) celui qui est connaturel et propre à chacun, qui maintient chaque partie de l'individu à sa place.

2) celui qui est commun — certains lieux étant plus communs que d'autres (la terre est le lieu de tous les animaux, l'air d'une partie seulement, etc.).

Dans la définition de Simplicius, le lieu et le temps possèdent la capacité active, enveloppante et définitionnelle. Ils constituent donc plus qu'une simple limite. Simplicius déploie donc contre Jamblique cette théorie qu'il croit inscrite chez ses autorités Aristote et Archytas. Il produit ainsi une part de nouveauté, une distinction conceptuelle entre deux formes de temps et de lieu.

\section{Les stratégies de conciliation}

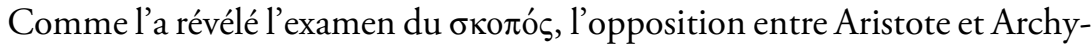
tas relève souvent d'une différence de points de vue: ils suivent des objectifs 
didactiques distincts, en vertu desquels ils adoptent une perspective soit logique soit ontologique. Or, lorsque les deux auteurs ne s'expriment pas d'une seule voix, l'exégète doit soit chercher le moyen de les réconcilier, soit trancher le débat en faveur de l'un des deux.

Le premier désaccord notable concerne les espèces de l'ov̉oía, dont les découpages respectifs témoignent d'ambitions divergentes. Le tableau suivant présente les divisions que Simplicius rapporte, en regard de ce que donnent les textes originaux $(77,4-11)^{44}$ :

\begin{tabular}{|c|c|c|c|}
\hline Archytas chez Simplicius & Archytas & $\begin{array}{l}\text { Aristote chez } \\
\text { Simplicius }\end{array}$ & $\begin{array}{c}\text { Arist., Mét. } \Lambda \text { 6, } \\
\text { 1071b3-11 }\end{array}$ \\
\hline 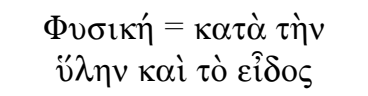 & 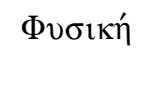 & 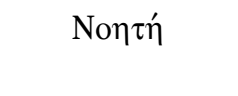 & 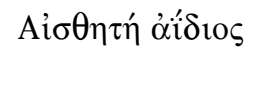 \\
\hline $\operatorname{Ai} \sigma \theta \eta \tau \eta^{\prime}=\sigma v^{\prime} v \varepsilon \tau \sigma \varsigma$ & Air $\theta \eta \tau \eta^{\prime}$ & $\operatorname{Ai\sigma } \theta \eta \tau \eta^{\prime}$ & 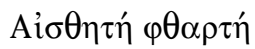 \\
\hline 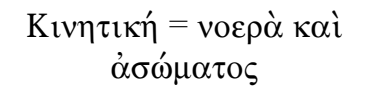 & & 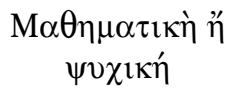 & 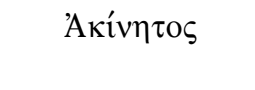 \\
\hline
\end{tabular}

Ce tableau montre les libertés que prend Simplicius (ou sa source, Jamblique) à l'égard de ses autorités. Il apparaît comme le véritable auteur de la triple division attribuée à Archytas qui, dans les fragments, ne distingue que deux substances-physique et sensible. De même, dans le passage parallèle de la Métaphysique, les trois substances reçoivent des noms différents de ceux mentionnés par l'exégète. D'une part, Simplicius introduit un troisième terme dans les propos d'Archytas, afin d'assurer la correspondance numérique avec Aristote. D'autre part, il modifie les noms pour améliorer la mise en relation. Or, malgré ces efforts, les deux divisions ne se rejoignent que sur la substance sensible. Comment dès lors les recouper? Simplicius les superpose, identifiant la substance physique à celle qui est relative à la forme et à la matière, tandis qu'il fait se correspondre substance sensible et substance composée. Il introduit ensuite un troisième terme par rapport à la seconde division aristotélicienne (en matière, forme et composé des deux)—la substance incorporelle et intellective—, qu'il identifie à la substance cinétique, aboutissant ainsi à un système dont les éléments sont censés se répondre. Or il n’explique pas vraiment la relation entre ces

44) C. Luna étudie les sources de ce passage dans Hadot (2001) 763-782. 
deux divisions et nous pourrions nous demander si, par le biais d'Archytas, Simplicius ne cherche pas à rétablir le lien entre platonisme et aristotélisme: la distinction qu'il attribue à Aristote ne concerne en effet que les dimensions de la substance sensible. En introduisant les attributs intellectifs et incorporels, il restaurerait l'idée de l'intelligible à côté de la perspective naturelle et sensible que suit Aristote.

Un peu plus loin, Simplicius fait état de la division aristotélicienne entre substance première et substance seconde. Il la juge irrecevable pour des Pythagoriciens car, en appelant première l'ov̉oía individuelle et seconde l'ov̉oí $\alpha$ générique, elle renverse l'ordre d'universalité et de souveraineté. Pour cette raison, selon lui, Archytas aurait préféré la division triple en matière, forme et composé-division par ailleurs connue d'Aristote mais inexploitée à cet endroit. À nouveau, la nuance révèle une différence d'optique. Dans la mesure où Archytas donne la division qui se retrouve dans la Métaphysique et dans la Physique, il se place du côté de l'ontologie ${ }^{45}$. Quant à Aristote, il se range dans la logique, en vertu de l'ordre pédagogique qu'il suit dans les Catégories ${ }^{46}$. En distinguant entre substance première et substance seconde, les Catégories usent d'un langage accessible et commun, qui correspond à un traité introductif et auquel la multitude est habituée $(78,31-79,5)$. En revanche, la distinction entre matière, forme et composé présente chez Archytas ne convient pas à des néophytes.

C'est pour la même raison que Simplicius prête à Aristote une perspective linguistique, là où il attribue à Archytas un regard ontologique. L'opposition devient flagrante dans l'examen de la nature des relatifs. Pour Aristote, dans un premier temps du moins, leur réciprocité ressortit au discours: "Tous les relatifs se disent par rapport à des réciproques (Cat., 7, 6 b 28)". Pour Archytas en revanche, la réciprocité dépasse la relation linguistique. Suivi par Simplicius, il insiste sur la réalité des relatifs. Ils sont

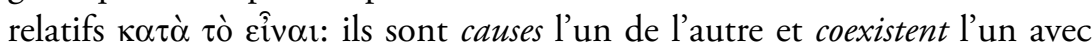
l'autre. Dans ces conditions, deux choses sont dites relatives parce qu'elles le sont, et non l'inverse: c'est seulement à partir du moment où une chose existe que se produit une distinction générique ${ }^{47}$. En d'autres termes, la

45) Métaphysique Z 3, 1029 a 2-7 et 26-33; Z 10, 1035 a 1-2; H 1, 1042 a 26-30; $\Lambda$ 3, 1070 a 9-13. La référence à la Physique est en revanche moins évidente.

46) In Cat., 91, 14-33. Ce passage est traduit et commenté par Hoffmann (2000) 361-362.

47) Cf. In Cat., 183, 2-10 et 189, 12-16. Ces passages ont été traduits dans l'examen du бколо́s. 


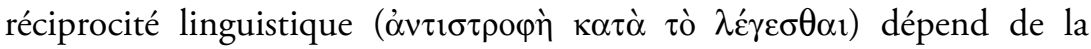

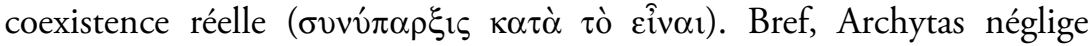

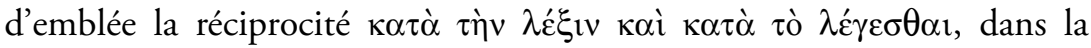

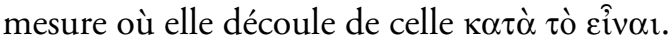

Or, plus loin, Aristote ne parle plus des relatifs en termes d'être dit, mais d'être, ce qui écarte nombre d'apories (Cat., 8 a 31-33). Il renverse la perspective, ne montrant plus l'être à partir de l'être-dit, mais l'être-dit à partir de l'être. Il passe d'un point de vue évident pour tous à un point de vue plus logique et plus causal. La différence entre Archytas et Aristote apparait à nouveau à Simplicius comme une différence de pertinence: le premier détient d'emblée la solution qui évite les apories et permet d'étendre aux réciproques des résultats que le second n'atteint qu'au gré d'hésitations, à propos des relatifs seulement ${ }^{48}$.

En général attentif aux discordances, Simplicius manque parfois une opposition qui ressort pourtant de ses citations et de ses explications. Chez Aristote, les relatifs ont pour propre "le fait d'être naturellement simultanés" ( tas, leur simultanéité ne se limite pas à leur coexistence mais implique leur causalité réciproque. L’opposition saute aux yeux:

Sont par nature simultanées toutes les choses qui impliquent réciproquement la conséquence de leur être, mais dont ni l'une ni l'autre n'est cause de l'être de sa réciproque. Par exemple, le double et le demi sont réciproques, car s'il y a double, il y a demi; s'il y a demi, il y a double. Aucun cependant n'est cause pour l'autre de son être (Cat., 13, 14 b 27-32. Cf. 15 a 8-12).

D'après Aristote, les relatifs s'impliquent réciproquement. Il exclut cependant qu'ils puissent être causes l'un de l'autre car, à ses yeux, la cause doit nécessairement être antérieure à l'effet et le premier terme doit toujours exister préalablement au second. Par conséquent, la relation de causalité exclut la simultanéité. Pour Archytas, la simultanéité possède un sens plus

48) C'est de cette façon que Simplicius répond à Syrianus, qui impute à la perspective linguistique d'Aristote une confusion sur la réalité des relatifs. Cf. In Cat., 199, 17-200, 3: "La différence tient peut-être ('ڤ $\sigma \omega \varsigma)$ en ceci, que la première [définition] indiquait l'être à partir

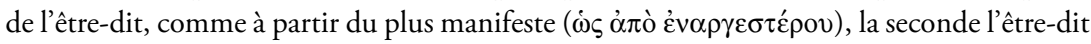

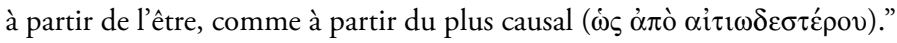


fort: chacun des termes fait exister et est la cause de l'autre. En effet, les relatifs ont pour propre de

coexister simultanément l'un avec l'autre et d'être causes l'un de l'autre ( $\tau$ '́

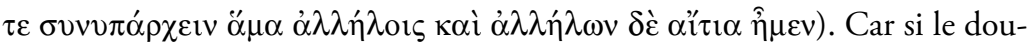
ble est, il est nécessaire que le demi soit également, et si le demi est, il est nécessaire que le double soit aussi. Et il est également nécessaire que le double soit cause du demi et que le demi soit cause du double $(189,23-27)$.

Simplicius passe à côté de ce désaccord au point de répéter, au terme de ce lemme, l'harmonie entre Aristote et Archytas, en y incluant également Platon $^{49}$ :

C'est donc avec raison qu'ils coexistent et disparaissent simultanément, et que l'un n'est pas sans l'autre, aussi bien selon Archytas, Platon et l'exposé d'Aristote dans des écrits plus précis $(196,30-33)^{50}$.

Les trois auteurs semblent s'accorder de façon indéniable sur la coexistence des relatifs. Mais Simplicius omet le problème issu de la causalité réciproque. En dépit de ses scrupules, notre commentateur connaît certaines failles.

La division du $\pi \alpha \dot{\alpha} \theta$ os offre une situation similaire $(331,26-332,5)$. Absente des Catégories, elle se trouve dans la Métaphysique:

On appelle affection, en un premier sens, la qualité suivant laquelle un être peut être altéré; par exemple, le blanc et le noir, le doux et l'amer, la pesanteur et la légèreté, et autres déterminations de ce genre.-En un autre sens, c'est l'acte de ces qualités et dès lors les altérations elles-mêmes.-On entend aussi par là, particulièrement, les altérations et les mouvements nuisibles, et surtout les dommages fâcheux.-Enfin, on appelle affections de grandes et cruelles infortunes (Métaphysique $\Delta 21,1022$ b 15-21; tr. Tricot).

49) Cf. République, IV, 438 b-439 a: Tout relatif l'est toujours par rapport à un objet déterminé qui le détermine en retour. Une chose n'est plus grande que par rapport à une autre plus petite, une science déterminée est science d'un objet particulier lui-même déterminé.

50) En 194, 4, Simplicius renvoyait aux propos meilleurs et plus élaborés de la Métaphysique ( $\Gamma$ 5, 1010 b 31-1011 a 2): si, en l'absence de science et de sensation, les substrats continuent d'exister, ils ne sont cependant plus sensibles ni connaissables. 
Citant ce passage, Simplicius ne peut s'empêcher de relever des différences par rapport à la division d'Archytas. Face à cette difficulté, il renomme les

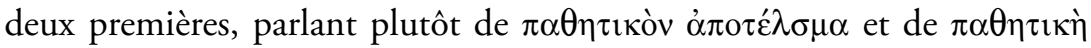

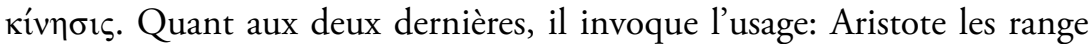

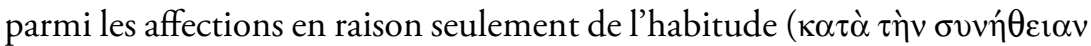

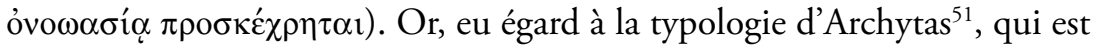
celle à laquelle fait droit Simplicius, Aristote ne distingue que les $\pi \varepsilon \pi$ ov $\theta$, c'est-à-dire les affections insensibles et sans conscience qui se produisent

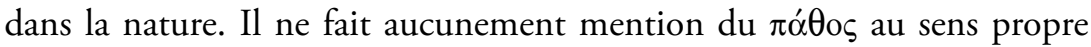
(l'effet de la sensation), du $\pi$ oín $\mu \alpha$ (l'adjuvant à l'achèvement de la substance), ni du $\pi \alpha \dot{\theta} \theta \eta \mu \alpha$ (l'affection inachevée). Simplicius ne note pourtant pas ce manque de complétude de la Métaphysique par rapport à la division d'Archytas. Sa vigilance est à nouveau réduite.

Bien qu'il paraisse acquis à la cause du pythagorisme, il arrive à Simplicius, en cas de litige, de donner raison à Aristote. Le cas le plus flagrant concerne le $\pi$ ov́ et le $\pi$ o $\varepsilon$, à propos desquels Simplicius fait état d'une opposition radicale entre Aristote et Archytas ${ }^{52}$.

Il faut savoir qu'Aristote, qui a posé le temps et le lieu dans la quantité, a fait

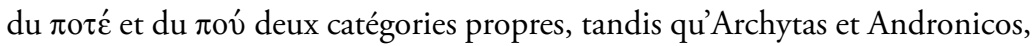

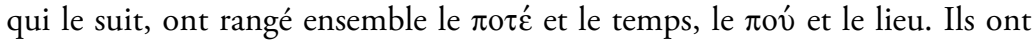
ainsi posé deux catégories et conservé la décade des genres. Les partisans d'Aristote doivent d'abord prouver que le lieu et le temps ressortissent à la quantité; ensuite que $\pi$ o $\tau \varepsilon ́$ et $\pi$ oú ne manifestent pas le temps et le lieu, mais une relation au temps et au lieu; enfin qu'ils méritent d'être comptés parmi les termes généralissimes $(342,21-28)^{53}$.

Simplicius explique que, dans leur conception de la catégorie de la temporalité, Andronicos et Archytas posent une nature particulière du

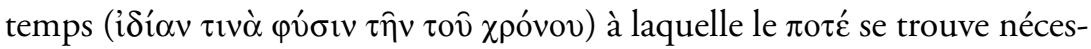
sairement lié, dans la mesure où la situation temporelle n'existe qu'eu

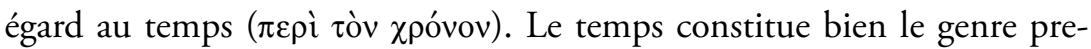

\footnotetext{
51) La division d'Archytas est citée dans la longue note 41.

52) Cette question a été envisagée par Hoffmann (2000) 363-364.

53) Simplicius signale que Plotin a nié le $\pi$ ov́ et le $\pi$ o $\tau \dot{\varepsilon}$, pour les avoir rangés dans le lieu et dans le temps. Or ces derniers ressortissent eux-mêmes à la quantité car il s'agit de mesures, voire de mesurés $(347,15-17 ; 358,8-11)$.
} 
mier à partir duquel toutes les déterminations temporelles peuvent exister: le quand n'a de sens qu'à être rapporté à la succession temporelle. Par conséquent, toute temporalité située s'avère seconde vis-à-vis du temps pris en lui-même (347, 6-12). Archytas et Andronicos ont donc privilégié

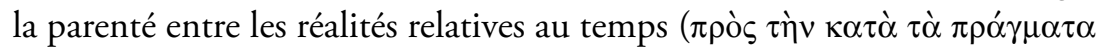

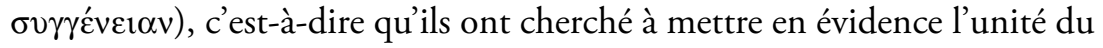
concept de temps, dont l'instant déterminé n'est qu'une expression.

À l'inverse, Aristote porterait l'accent sur les différences de signification

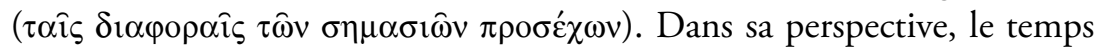
apparaîtrait comme une quantité continue et mesurable. En revanche, différent du temps ou d'une de ses parties, l'objet se trouverait en relation à son égard ( $\sigma \chi \varepsilon \dot{\varepsilon} \sigma 1 \varsigma \pi \rho o ̀ \varsigma \chi \rho o ́ v o v)$ et, du même coup, serait dit positionné dans le temps ( $\dot{\varepsilon} \vee \chi \rho o ́ v \omega)$. La réponse prêtée à Aristote permet de résoudre la première objection : le temps peut faire l'objet d'une mesure. Par conséquent, il relève de la quantité. De plus, la distinction répond également à la deuxième objection: si l'objet situé dans le temps s'avère différent du temps, il possède seulement une relation avec lui (346, 1-347, 5). En clair, nous retrouvons sur ce point l'opposition apparue dans la détermination du бколós: là où Archytas adopte une démarche conceptuelle qui se concentre sur le genre dans sa pureté et son unité, Aristote se montre attentif à des considérations linguistiques.

La troisième objection soulève davantage de difficultés, comme en témoigne Plotin ${ }^{54}$ :

Si ce qui est dans le temps est quelque chose d'autre que le temps, et si ce qui est dans un lieu est quelque chose d'autre que le lieu, pourquoi "ce qui est dans un vase" ne serait-il pas une nouvelle catégorie, ce qui est dans la matière une autre et ce qui est dans un substrat une autre encore? Et pourquoi n'en irait-il pas de même pour la partie dans le tout, le tout dans les parties, le genre dans les espèces, et l'espèce dans le genre? Et ainsi nous multiplierions les catégories.

Si le $\pi$ ov́ et le $\pi$ o $\varepsilon \varepsilon$ désignent des relations au lieu et au temps, en vertu de quoi posséderaient-ils un statut isolé parmi les onze façons possibles d'être dans quelque chose $(348,27-35)^{55}$ ? Pour quelle raison, parmi les neuf

54) Plotin, VI, 1, 14, 19-24 (tr. Luc Brisson). Le texte est cité par Simplicius, In Cat., 349, 5-10.

55) Simplicius reprend la liste des significations dressée par Aristote dans la Physique (IV, 3, 
autres façons, aucune n'engendrerait d'autres catégories? Simplicius rapporte en premier lieu la solution de Jamblique: toutes les autres formes d'être dans quelque chose s'avèrent reconductibles à la relation et aux relatifs. Peu satisfait par cette solution parce qu'elle ne définit pas suffisamment en quoi le $\pi$ ov́ et le $\pi$ o $\tilde{\varepsilon}$ se distingueraient des relatifs, Simplicius y voit plutôt deux formes différentes de relatifs, considérant que tout ce qui est dans quelque chose ne mérite pas de faire l'objet d'une catégorie propre. Afin de produire un critère clair de démarcation, il définit trois conditions ${ }^{56}$ :

1) Il faut que l'un soit contenant, l'autre contenu.

2) Chaque composant doit conserver sa nature propre, sans devenir partie ou constituant de l'autre dans lequel il se trouve.

3) L'universel doit exister absolument; par exemple, le corps est ce qui se trouve dans le lieu et dans le temps.

Dans la mesure où les neuf autres significations font apparaitre des relations constitutives et non autonomes, ou encore qui ne peuvent subsister séparément, elles ne satisfont pas aux trois critères précédents. Par exemple, l'espèce est dans le genre, mais chacun des deux termes ne possède qu'une existence notionnelle. Par conséquent, leur séparation est loin d'être absolue. En revanche, les choses qui se trouvent dans le temps ou dans le lieu sont bien différentes du temps et du lieu, puisqu'elles conservent leur nature propre (350, 3-9).

Simplicius admire l'à $\gamma \chi \chi^{\prime} v o 1 \alpha$ d'Aristote qui, par la discrimination qu'il établit entre le temps et le $\pi$ o $\tilde{\varepsilon}$, le lieu et le $\pi$ ov́, a réalisé un véritable progrès philosophique vis-à-vis d'Archytas $(348,25)$. Néanmoins, la distinc-

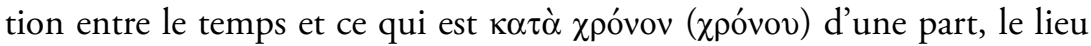

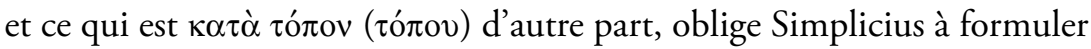
une théorie personnelle de la relation, afin de comprendre ce qui fait la spécificité de ces deux catégories par rapport aux autres formes de l'être dans quelque chose. Il doit donc produire des critères de démarcation à l'intérieur de ce genre, afin de séparer ce qui pourrait faire l'objet d'une

210 a 14-24), mais lui en ajoute deux: d'une part le sens d'accident dans la substance et, de façon générale, d'être dans un substrat, d'autre part le fait d'être dans le temps.

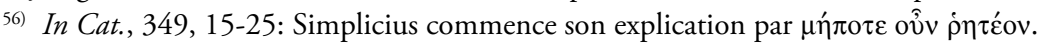


catégorie propre de ce qui doit appartenir aux relatifs. Face à de tels désaccords, Simplicius s'attèle à résoudre la symphonie des deux auteurs. Il en ressort qu'il ne se comporte pas en partisan définitivement acquis. Il se livre à une mise à l'épreuve des concepts qui, dans le dernier cas, tourne à l'avantage d'Aristote, dont il souligne le mérite. Cet exercice le contraint aussi à se démarquer de Jamblique et d'autres exégètes, afin d'élaborer une théorie personnelle, utile pour l'examen du réel.

En d'autres lieux, la confrontation avec Archytas constitue moins une cause de dysharmonie qu'un moyen de réconcilier les Catégories et certains aspects incompatibles du platonisme. Simplicius convoque Archytas à propos de la notion de figure $(\sigma \chi \eta \hat{\mu} \mu \alpha)$, sous le prétexte qu'il tient sur le sujet une position plus claire qu'Aristote. Notons que cette clarté demeure somme toute très relative, dans la mesure où elle n'appartient qu'au travail de notre commentateur ${ }^{57}$.

La polémique s'enracine dans un désaccord entre Platon et Aristote sur le statut de la figure: est-elle antérieure ou postérieure au corps dans lequel elle apparaît? Pour Platon, les $\sigma \chi \eta \dot{\mu} \mu \alpha \alpha$ constituent les principes des éléments et sont antérieurs aux corps: le chaud est composé de figures à angles aigus, telles des pyramides, le froid de figures moins aigues, tel l'icosaè$\mathrm{dre}^{58}$. Ces figures s'avèrent matérielles, naturelles et en mouvement. Elles se distinguent des figures mathématiques qui, pour leur part, sont immobiles et immatérielles. Or Aristote ne s'accorde ni avec Platon ni avec les mathématiciens, car il pose des figures matérielles qui coexistent aux corps, ni non plus avec les Stoïciens, qui jugent les figures corporelles et les identifient à des qualités. En résumé, et bien que nous ne lisions rien de tout cela dans les Catégories, Simplicius attribue à Aristote une position intermédiaire entre Platon et les Stoïciens selon laquelle, sans être ni matérielle ni antérieure aux corps, la figure se produirait simultanément dans les corps engendrés.

Le problème rencontré par Simplicius, et par Jamblique avant lui, consiste à réconcilier Platon et Aristote qui, pensent-il, ne peuvent soutenir des thèses radicalement distinctes. Pour cette raison, il invoque l'autorité d'Archytas, qui aurait au préalable clarifié la question en opérant une

57) In Cat., 271, 6-272, 6. Ni dans les Notions universelles ni dans les Catégories nous ne trouvons la trace des thèses discutées par Simplicius.

58) In Cat., 271, 9-14. Cf. Timée, 55 d-56 b. 
distinction entre $\sigma \chi \eta \hat{\mu} \mu \alpha$ et $\sigma \chi \eta \mu \alpha \tau \imath \sigma \mu o ́ s$, entre la figure et le résultat de la configuration. Ce serait au niveau du $\sigma \chi \eta \mu \alpha \tau \imath \sigma \mu o ́ s$, et non du $\sigma \chi \hat{\eta} \mu \alpha$, que se situerait la qualité formelle. La qualité qui exprime la figure se produit en effet dans les corps en tant qu'ils sont déjà en train de se configurer $(\mu \varepsilon \tau \alpha \sigma \chi \eta \mu \alpha \tau i \zeta o ́ \mu \varepsilon v \alpha)$. Il en résulte une distinction entre le corps en train de se configurer et la qualité qui s'y établit: la limite formelle apparaît au moment du façonnement. De plus, les figures ne traversent pas l'ensemble du corps, mais demeurent en surface: ce qui possède la figure, sans être la figure elle-même, la reçoit de l'extérieur et se trouve embrassé par elle. En outre, ces qualités n'existent pas en acte à partir d'elles-mêmes

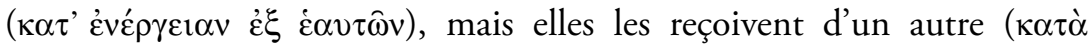

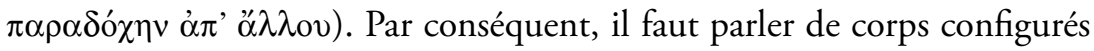
et non configurant.

Dans cette interprétation d'Archytas, les $\sigma \chi \eta \eta_{\mu \alpha} \alpha \alpha$ existent antérieurement aux corps, sans être encore des qualités ${ }^{59}$. Il convient seulement de parler de qualités à partir du moment où il est question de corps en train de

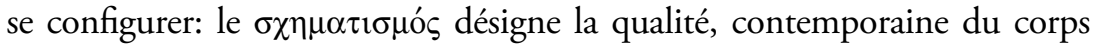
configuré. En ce sens, la doctrine archytéenne manifeste une parfaite harmonie avec la position d'Aristote dans les Catégories. Par ailleurs, l'idée de don extérieur qu'elle recèle s'accorde pleinement avec la théorie de Platon, puisque les corps élémentaires fournissent les formes aux autres corps sensibles. La figure se révèle une qualité extrinsèque et superficielle. Par conséquent, cette interprétation, qui distingue entre la figure mathématique, la figure informante et la figure informée, explique l'avènement de la forme dans le corps, tout en restaurant la symphonie d'Aristote et de Platon ${ }^{60}$.

Dernier cas de figure, plus rare: Simplicius renvoie ses deux autorités à une erreur commune. Il devient alors l'auteur de la précision qui doit lever l'ambiguïté du langage ordinaire dont elles ont toutes deux été la victime:

\footnotetext{
59) Ce vocabulaire est évidemment absent du texte de Platon.

60) Simplicius utilise aussi Archytas pour harmoniser Aristote et le néoplatonisme, afin d'introduire la doctrine du $\lambda$ ó $\gamma$ os dans les choses au sein des Catégories (290, 11-291, 18). Grâce à une citation d'Archytas, il affirme que les variations qualitatives (positives ou négatives) dépendent du $\lambda$ ó $\gamma$ os de la chose, et non de sa participation à la forme. En d'autres termes, la

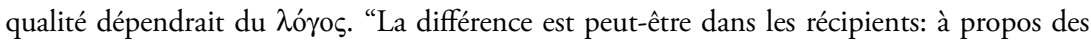
couleurs connaturelles, par exemple le blanc dans le lait ou dans la neige, il est évident que l'excès positif est conformément au logos naturel lui-même" (290, 23-25).
} 
Mais peut-être, même s'il est possible d'observer la propriété des relatifs au singulier $(\dot{\varepsilon} v \imath \kappa \hat{\omega} \varsigma)$, de la même façon que nous observons la propriété unique du trois et de chacun des nombres qui subsistent dans la pluralité, et même s'il est possible aussi dans le cas de cette catégorie, comme dans le cas des autres, de s'exprimer tant au singulier qu'au pluriel, ce n'est pourtant pas de la même façon. Dans le cas des autres, en effet, l'un était le genre lui-même tandis que les multiples étaient les espèces circonscrites dans le genre, ici en revanche les multiples ne sont pas les espèces de ce qu'on appelle relatif, mais ce qui constitue la relation et dans quoi on observe la relation. Car c'est le propre de la relation seule d'exister uniquement dans les multiples, ce qui ne se présente pour aucune des autres catégories. Voilà donc, semble-t-il, ce qu'il faut conclure de ce qu'on vient de dire, à savoir que la relation possède une différence par rapport aux autres catégories en ce qu'elle ne peut se dire, à leur façon, au singulier et au pluriel, puisqu'elle possède absolument son existence dans la pluralité $(160,34-161,10)$.

À la différence des autres genres, il est impossible d'user du singulier et du pluriel à propos des $\pi \rho o ́ s \tau$, pour désigner alternativement le genre et la multiplicité. Pour cette catégorie, et ce contre l'usage tant d'Aristote que d'Archytas, le pluriel ne désigne pas les espèces du genre mais la pluralité des relatifs, puisque la relation possède la particularité d'exister seulement dans la pluralité (il faut toujours parler des relatifs, même lorsqu'on cite des exemples). Dans cette situation quelque peu singulière, Simplicius s'autorise à une prise de distance à l'égard de ses autorités, mais à la condition de nombreuses précautions. Il se livre à une critique qui repose sur une tentative de réconcilier non plus deux auteurs, sujets au même écueil, mais la langue philosophique et rigoureuse à la réalité de ce qu'elle décrit.

Les écarts inévitables entre Archytas et Aristote contraignent Simplicius à des exercices de funambulisme. Pour recouvrer l'équilibre, il use toutefois de plusieurs stratégies. La première consiste à renvoyer les désaccords à des divergences d'objectif: les deux auteurs ne diffèrent pas quant au fond-ce que notre exégète s'empresse de justifier par des références à d'autres œuvres d'Aristote—, mais quant au public ciblé. Si le Stagirite s'autorise la distinction, peu rigoureuse, entre substance première et substance seconde au lieu de la division en matérielle, formelle et composée, c'est parce qu'il s'adresse à des débutants, et non à des métaphysiciens confirmés. La deuxième stratégie de Simplicius l'incite à ignorer des oppositions manifestes. Si la chose ne relevait pas de la pure spéculation, il 
faudrait s'interroger sur la raison de ces silences: sont-il délibérés ou non? La dernière stratégie s'avère la plus féconde, étant donné qu'elle aboutit à la création de nouvelles doctrines. S'il serait ambitieux de parler de système inédit, il convient à tout le moins de souligner les mérites de Simplicius, qui fournit quelques critères efficaces, notamment pour comprendre la

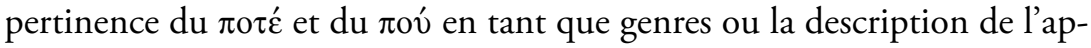
parition de la figure dans les corps.

\section{Une conciliation ouvrière de doctrine}

Pour le lecteur moderne, les discordances les plus intéressantes appartiennent à cette troisième catégorie, là où la résolution de l'opposition entre Aristote et Archytas aboutit à la production d'une doctrine nouvelle et originale, qu'elle soit empruntée à Jamblique ou, mieux, qu'elle naisse du commentaire de Jamblique mais soit imputable à Simplicius lui-même. Or, parmi les discordances autour de la doctrine des catégories, la situation de la quantité et, en particulier, la question de la poлń mériteraient une attention particulière. Simplicius note à ce sujet, entre ses autorités, un désaccord important qui concerne la catégorie dans son ensemble. Tandis que, dans les Catégories, Aristote distingue deux espèces—le continu d'une part, le discret de l'autre-, Archytas mentionne un troisième terme: la роли́. Littéralement, la poлń désigne l'inclinaison ou l'action d'incliner, en parlant notamment d'une balance dont les plateaux penchent en un sens ou en un autre. Elle signifie aussi l'impulsion de haut en bas, autant que ce qui détermine cette impulsion. Par extension, la porń s'identifie au poids, puisqu'elle correspond à la tendance d'un corps à se diriger vers le bas ${ }^{61}$.

Le désaccord apparaît à la simple confrontation des textes, puisque les Catégories donnent la division suivante:

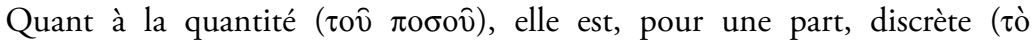

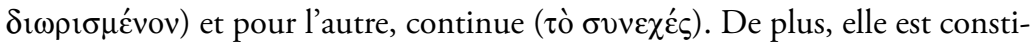
tuée ou bien de parties qui occupent une position les unes par rapport aux

${ }^{61)}$ La traduction de $\operatorname{\rho onń~par~inclinaison~doit~permettre~la~distinction~tant~avec~le~poids~}$

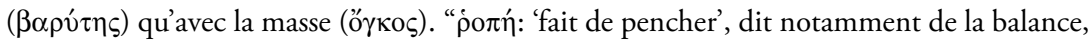
'balancement, poids qui fait pencher, influence décisive, crise'” (Chantraine 1999). 
autres dans les ensembles ou bien de parties qui n'occupent pas de position.

Or est discrète la quantité telle que le nombre ( $\alpha \rho \imath \mu \mu o ́ s)$ et le discours ( $\lambda$ ófos), alors que la quantité continue comprend la ligne ( $\gamma \rho \alpha \mu \mu \eta ́)$, la surface $(\dot{\varepsilon} \pi \imath \varphi \alpha ́ v \varepsilon \imath \alpha)$ et le corps $(\sigma \hat{\omega} \mu \alpha)$, mais aussi, en dehors de cela, le temps et le lieu (Cat., 6, 4 b 20-25; tr. Bodéüs 2001).

En revanche, pour ce qui est de la position d'Archytas, Simplicius rapporte ceci, au début de son explication du texte aristotélicien relatif à la quantité:

À mon avis, il faut prêter attention à Archytas, qui a lui-même divisé le combien ( ò $\pi$ roóv) en trois. Il écrit en effet ceci: "Les différences de la quantité sont au nombre de trois: la première réside dans l'inclinaison ( $\dot{\varepsilon} v$ jo $\pi \hat{\alpha})$, comme le talent, la deuxième dans la grandeur ( $\dot{\varepsilon} v \mu \varepsilon \gamma \dot{\varepsilon} \theta \varepsilon \mathrm{\varepsilon})$, comme de deux coudées, la troisième dans la pluralité ( $\left(\dot{\varepsilon} v \pi \lambda \alpha^{\prime} \theta \varepsilon \mathrm{l}\right)$, comme dix (In Cat., 128, 16-19)."

À la division aristotélicienne de la quantité en discret et en continu, Archytas en substitue une autre, distinguant entre l'inclinaison, la grandeur et la pluralité. De deux espèces chez Aristote il passe à trois. En outre, le changement de vocabulaire modifie la perspective: là où Aristote formule deux critères en vertu desquels se répartissent les espèces de la quantité, Archytas propose plutôt des espèces déjà formées. Enfin, il insiste sur sa nouvelle espèce, la jo

$\mathrm{Si}$ minime que semble la modification, elle altère radicalement la conception globale du genre. Introduire une espèce à côté du couple formé par le discret et le continu implique de le penser selon d'autres critères, dans la mesure où nous passons d'une bipolarité à un système à trois entrées. Il en résulte que l'opposition du discret et du continu doit soit être raffinée, soit être dépassée. En dehors des répercussions logiques, il faut considérer la théorie physique qui sous-tend la tripartition. Intégrer le poids dans la quantité entraîne une prise de distance par rapport à une conception purement qualitative de la physique. Si l'idée de poids n'appartient plus à la qualité mais à la quantité, elle glisse doucement d'un système régi par la notion de lieu naturel à un système où la gravité du corps reçoit une mesure-qui se révèle en outre indépendante de la grandeur et du nombre, s'agissant d'espèces différentes d'un même genre.

Confronté à cette opposition textuelle et face à une telle difficulté, le commentateur devra soit admettre une incompatibilité entre les deux 
autorités, soit produire une doctrine qui réconcilie les aspirations de chacune. Simplicius suivra cette seconde option.

\section{La thèse de Jamblique}

Simplicius signale que la solution d'Archytas-la subdivision supplémentaire de la quantité-a été reprise par Athénodore ${ }^{62}$ et par Ptolémée (128, $5-8)^{63}$. En rétablissant la chronologie, il ressort que la division triple du genre remonterait au moins aux débats postérieurs à Andronicos de Rhodes. Cependant, comme les travaux de ces auteurs n'ont pas subsisté, il est difficile d'identifier les raisons qui les ont menés à s'écarter d'Aristote. Il est en revanche plus intéressant d'étudier leur impact sur des commentateurs que nous connaissons davantage.

Avant d'interroger le bien-fondé de cette division, Simplicius rappelle que, dans la topologie d'Aristote, la lourdeur n'appartient pas à la quantité mais à la qualité, au même titre que la minceur et l'épaisseur (In Cat., 128, 8-12): une chose est lourde ou légère, autant que mince ou épaisse, en vertu du quel et non en vertu du combien. Il s'agit là d'affections des réalités, eu égard auxquelles elles sont qualifiées et se révèlent telles ou telles. À s'en tenir à de telles affirmations, la pesanteur s'avère sans lien avec la quantité, puisqu'elle désigne un état de la chose plutôt qu'elle n'en indique une mesure. Que dire dans ce cas de la mine ou du talent? Qu'une chose pèse deux mines ou trois talents semble bien identifier cette quantité à un nombre. Dès lors, le poids, exprimé en mine et en talent, devrait être ramené au nombre, puisqu'il s'agit de dire combien de mines pèse un objet. Mais qu'advient-il alors de la mine et du talent pris en eux-mêmes, c'est-à-dire

62) Simplicius se réfere à Athénodore à plusieurs reprises dans le Commentaire sur les Catégories, à qui il attribue un traité Contre les Catégories d'Aristote (62, 25-26). Dans la mesure où Simplicius fait également état du traité de Cornutus Contre Aristote et contre Athénodore, il en résulte qu'Athénodore serait antérieur à ce dernier. Or nous savons que Lucius Annaeus Cornutus aurait vécu au premier siècle PCN (en 65, il aurait été banni de Rome, où il enseignait, par Néron). Nous pouvons donc situer Athénodore entre Andronicos de Rhodes et le traité de Cornutus. D’après les renseignements de Simplicius, nous pouvons déduire que, tout comme Cornutus après lui, Athénodore était un partisan de l'interprétation linguistique des Catégories (Cf. In Cat., 159, 31-33; 187, 28-30). Sur ces deux auteurs, Moraux (1973) 585-601; Hadot (2001) 43 et 386-387.

63) Il s'agit de Claude Ptolémée, astronome, géographe et mathématicien grec ayant vécu en Égypte de 90 à 168. 
en tant qu'ils possèdent une pesanteur donnée, irréductible au nombre ou à la grandeur? S'il est possible d'établir le rapport numérique entre mine et talent, en disant que le talent équivaut à soixante mines, ce procédé aboutit à un cercle logique qui revient à définir une quantité par une autre, elle-même définie dans son rapport à la première. De plus, pourquoi en irait-il ainsi pour le poids et pas pour la grandeur: qu'une chose mesure trois coudées n'implique pas de réduire la longueur au nombre, si ce n'est

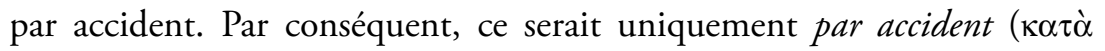
$\sigma v \mu \beta \varepsilon \beta \eta \kappa o ́ \varsigma)$ que le poids serait une quantité et s'exprimerait par le nombre ou la grandeur.

Contre cette difficulté, notre commentateur propose de postuler, avec le $\mu$ ท́ $\pi$ o $\varepsilon$ caractéristique de la prise de parole, que

ce n'est peut-être pas au sens où le blanc est un combien par accident-parce que la surface est un combien-, que l'est l'inclinaison, mais elle est un combien par elle-même, pour la raison qu'elle admet par elle-même le propre du combien, le fait d'être égal et inégal, au même titre que d'autres déterminations admettent l'excès et le défaut (In Cat., 128, 13-16).

Mine et talent répondent au propre de la quantité tel qu'Aristote le définit, l'égal et l'inégal, et non au propre de la qualité, le plus et le moins ${ }^{64}$. La mine s'avère égale à la mine, le talent au talent. Ils produisent respectivement une même inclinaison de la balance qui, de ce fait, reste à l'horizontale et demeure stable. Dès lors, il y a bien lieu d'instituer une troisième subdivision de la quantité, qui décrit une troisième façon d'être égal ou inégal (dont il restera à énoncer la différence) ${ }^{65}$. La ṕo $\eta_{\eta}$ n’est donc pas quantité par accident, mais bien par soi.

Jamblique avait déjà accepté la division attribuée à Archytas et l'avait intégrée à son exégèse des Catégories. Simplicius rapporte son avis, avec

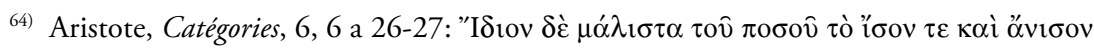
$\lambda \varepsilon ́ \gamma \varepsilon \sigma \theta \alpha$.

65) Simplicius reprendra cette conclusion plus loin, sans guère mentionner Jamblique: "Archytas quant à lui, qui affirme que le fait d'être égal ou inégal est propre au combien, soutient qu'il s'observe dans la pluralité, dans la grandeur et dans l'inclinaison, mais qu'aucun d'entre eux n'accompagne ni la substance, ni la qualité. C’est donc en trois sens qu'il définit le fait d'être égal et inégal, selon les trois différences du combien (151, 32-35).” 
lequel il semble lui-même s'accorder, dans la mesure où il résoudrait un problème rencontré $(128,20-22)$ :

Jamblique admet cette division, étant donné que cette triade est conçue selon la mesure la plus parfaite de la quantité et qu'elle s'accorde avec les choses ( $\dot{\omega} \varsigma$

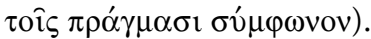

La rectitude de la solution jamblichéenne résulte de l'harmonie qu'elle manifeste. D'une part elle s'accorde avec Archytas, restaurant l'autorité de la doctrine pythagoricienne et révélant la vérité fondamentale de la tradition grecque. D'autre part elle concorde avec les choses mêmes puisque, comme l'a souligné Simplicius, elle épuise le genre de la quantité-obéissant en cela au critère de la méthode pythagoricienne: s'accorder avec les réalités. Elle manifeste donc bien les différentes façons pour une chose d'être quantifiée.

Comment, dès lors, Jamblique justifie-t-il l'introduction de la po $\pi \eta$ ? En quoi se trouve-t-elle en harmonie avec les réalités? Simplicius cite in extenso son explication:

La quantité selon l'inclinaison n'est identique ni à la grandeur ni à la pluralité. Elle s'observe plutôt dans le mouvement et possède le combien selon le poids et la légèreté (128, 22-24).

Les attributs de la po $\pi \eta$-le fait d'être observée dans le mouvement et la pesanteur-la lient plus que les autres espèces, nombre et longueur, au domaine de la physique. Sans qu'il ne le fasse ici, Simplicius pourrait donc invoquer cette raison pour justifier qu'Aristote n'en traite pas dans les Catégories, ouvrage de logique et non de physique. Jamblique poursuit:

A donc été laissée de côté cette division, qui se donne comme suit: 'parmi les quantités, les unes possèdent une inclinaison les autres non'. Il est clair que cette dernière n'appartient ni aux continus ni aux discrets, ni à ce qui a ou non une position (128, 24-27).

Jamblique estime qu'Aristote a manqué d'exhaustivité mais qu'il suffit de restituer ce qu'il a laissé de côté. Il suggère de compléter la division aristotélicienne de la quantité, en ajoutant-à côté des oppositions entre le continu et le discret d'une part, entre ce qui a une position et ce qui n'en a pas d'autre part-l'opposition entre ce qui possède une inclinaison et ce 
qui n'en a pas. Inspiré par Archytas, ce geste s'inscrit bien dans la ligne des Catégories: il fournit un troisième critère de la quantité, plutôt qu'il n'en isole une espèce supplémentaire. Dans les Catégories, les mêmes exemples servent en effet à illustrer les deux premières oppositions: la ligne est une quantité continue dont les parties occupent une position, tandis que le nombre est une quantité discrète dont les parties n'occupent pas de position déterminée. Ces couples ne mènent donc pas à la formation d'espèces proprement dites, pour lesquelles les membres de l'une ne pourraient également appartenir à l'autre, mais elles donnent deux angles distincts sous lesquels envisager les exemples. À l'inverse, dans le traité Sur les notions universelles et sa lecture par Simplicius, la grandeur (la ligne) et la pluralité (le nombre) constituent bien des espèces. Bref, Jamblique recoupe les démarches respectives des deux autorités: identifier des critères pour Aristote, isoler des espèces pour Archytas.

Et dans l'univers ${ }^{66}$, continue Jamblique, cette division paraît manifeste, puisque d'une part les quatre éléments ont une inclinaison, d'autre part le ciel est sans inclinaison $(128,27-28)$.

Jamblique utilise un argument physique fondé empiriquement pour prouver la nécessité de la division logique. À l'intérieur de l'univers, les éléments observent des inclinaisons qui, faut-il le préciser, s'avèrent différentes-puisque la terre et l'eau possèdent davantage de lourdeur, l'air et le feu davantage de légèreté. Le ciel, en revanche, n’a aucune inclinaison: il ne tend ni à s'éloigner de la Terre ni à s'effondrer sur elle. D'après Jamblique, il en résulte que la jo $\pi$ ń apparaît comme la différence propre au terrestre, qui s'exprime dans la quantité, là où les deux autres espèces de la quantité s'appliquent aussi au céleste. Par conséquent, l'inclinaison permet d'établir la distinction, au sein du domaine de la physique, entre le monde céleste et le monde terrestre. Cela suffit-il à affirmer que la différence physique est d'ordre quantitative et non qualitative?

60) 'Ev $\tau \hat{̣} \pi \alpha v \tau i$ : Jamblique postule qu'Archytas écrit sur la totalité des réalités ( $\pi \rho \alpha ́ \gamma \mu \alpha \tau \alpha)$, et pas seulement sur les termes qui les désignent-ce qui explique par ailleurs un des titres mentionnés par Simplicius. 
Et au sein des mouvements, les mouvements rectilignes se produisent avec l'inclinaison, eux qui possèdent un début, une fin et marquent entre eux des intervalles de repos. En revanche, le mouvement circulaire, qui est continu et sans début ni fin, dans la mesure où il est mouvement perpétuel, est sans inclinaison (’’ $\rho \rho \varepsilon \pi \dot{n} \varsigma ; 128,29-32)$.

Conséquence du point précédent, si la po $\pi$ ń s'observe dans le mouvement, elle n'appartient pas à toutes ses formes, car elle est propre au mouvement rectiligne, qui caractérise le monde d'ici-bas. À nouveau, elle apparaît comme un critère distinctif entre deux mondes, mais aussi entre les deux sortes de mouvement qui leur correspondent.

Dans les combien incorporels, dit-il, une telle différence est évidente: si on admet en principe que l'âme est un combien en soi, en tant qu' elle incline vers

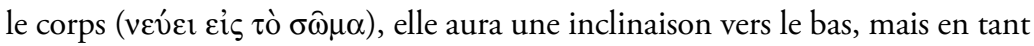
qu'elle se détourne des choses d'en bas et incline vers l'intelligible ( $\pi \rho$ ò $\varsigma$ iò

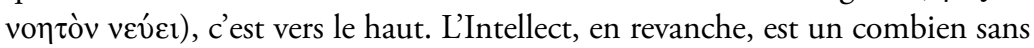

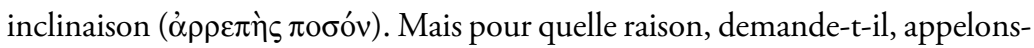
nous combien les intervalles de la voix ( $\tau \dot{\alpha} \delta 1 \alpha \sigma \tau \dot{\eta} \mu \alpha \tau \alpha \tau \hat{\eta} \varsigma \varphi \omega \vee \eta ิ \varsigma)$, tandis que nous n'en faisons pas autant pour les intervalles de l'inclinaison (128, $32-129,1)$ ?

La théorie de l'inclinaison de l'âme se trouve déjà chez Plotin. Dernière

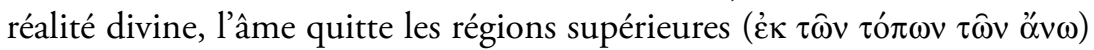

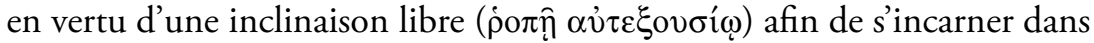
les corps, d'y exercer sa puissance et d'ordonner les réalités postérieures ${ }^{67}$. Cette descente révèle les capacités psychiques, qui demeurent inactives et inconnues de l'âme elle-même aussi longtemps que celle-ci reste dans l'incorporel où elle n'a pas l'occasion de les mettre en pratique. Or ce séjour corporel s'avère cause de dommages pour l'âme, qui doit s'enfuir aussitôt après y avoir exercé ses facultés. Comment dès lors concevoir le lien somato-psychique et la possibilité du retour de l'âme? Plotin développe ce point dans son avant-dernier traité6 ${ }^{68}$ : en réalité, ce n'est pas l'âme ellemême qui se mêle au corps dans la descente, mais son ombre; car, pour l'âme, s'incliner vers le bas, c'est regarder vers le pire-vers ce qui est péris-

67) Plotin, Enn., IV 8 [6] 5, 24-37.

68) Plotin, Enn., I, 1 [53] 12, 23-28. 
sable et relève du sensible ${ }^{69}$. L'âme elle-même ne descend pas: son inclinai-

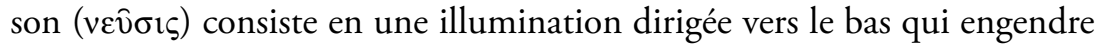
un reflet et fait vivre ce qui est illuminé ${ }^{70}$. L'âme doit plutôt s'incliner vers le haut et exprimer le désir qu'elle éprouve envers l'Intellect. À cette seule condition, elle peut demeurer parfaite et toujours pure, sans jamais incliner vers le pire. Tournée dans cette direction, elle en arrive à oublier les sensibles et à disparaître de ce qu' elle illumine ${ }^{71}$. Si Plotin évoque une inclinaison psychique tant vers le bas (les corps qui entraînent la po $\pi \eta$ ) que vers le haut (l'Intellect), il ne la traite en rien en termes de quantité, sinon par métaphore-ajoutons que, dans le cas où l'âme ne choisit de tendre ni vers le bas ni vers le haut, elle observe un mouvement circulaire dépourvu de

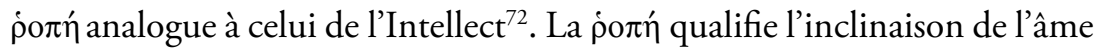
vers le bas, c'est-à-dire vers ce qui est ontologiquement postérieur à l'intelligible. Or c'est en toute liberté, de façon indépendante et souveraine, que l'âme incline vers les corps, et non en raison d'une contrainte physique à laquelle elle serait nécessairement soumise.

Dans l'argument de Jamblique, la distinction entre deux types de réalités corporelles entraine une analogie avec les réalités incorporelles: de

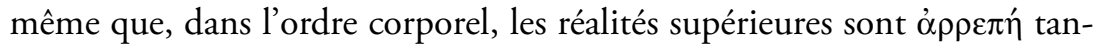
dis que les réalités inférieures possèdent une po $\pi$ ๆ́, dans l'ordre incorporel l'Intellect ne possède pas de $\dot{\rho}$ orń, alors que l'âme en possède une en vertu de sa descente dans le corps. La circularité du mouvement de l'Intellect le rapproche des réalités célestes, là où l'âme descend (ou monte) en ligne

69) Cf. Enn., V 3 [49] 9, 1-7. Pour Plotin, poлń désigne aussi la faible impulsion selon laquelle un individu de rang inférieur dévie du droit chemin (III 3 [48] 4, 45-50). L'idée de lourdeur du corps qui entraîne l'âme à sa suite se trouve déjà chez Platon: une légère impulsion externe suffit à rendre malade un corps débile (République, VIII, 556e); un individu moins noble, pris à des occupations moins élevées (recherche des honneurs ou des plaisirs), risque fortement d'incliner vers le vice, donc à abîmer son âme à cause d'une tendance vers le corps (Lois, XI, 920b). Notons que, chez Aristote, le plaisir et la douleur pèsent lourdement sur la poursuite de la vertu et du bonheur, en traversant la vie de part en part (Éthique à Nicomaque, X, 1, 1072 a 24-28).

70) Comme le souligne Aubry (2004) 320-322. Voir également le propos de Plotin Contre les Gnostiques (II 9 [33] 4, 7-9): “la cause productrice du monde n'est pas que l'âme s'incline, mais plutôt qu'elle ne s'incline pas; car s'incliner, c'est oublier les intelligibles; et si elle les avait oubliés, comment façonnerait-elle le monde?”

71) Voir Enn. I 8 [51] 4, 25-32; 15, 20-23; IV 4 [28] 25, 2-8.

72) Plotin, Enn., II 1 [40] 3, 22-23. 
droite. Par conséquent, la doctrine de la potń sert à rendre compte du double mouvement rectiligne de l'âme-l'un ascendant, la conversion

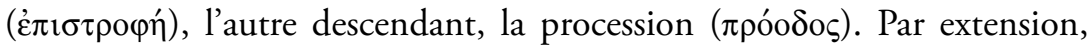
elle justifie aussi la différence entre deux formes de connaissance: l'une circulaire, la connaissance intelligible d'une pensée qui se pense elle-même, l'autre rectiligne, qui vise intentionnellement des objets vers lesquels elle se projette.

Jamblique établit une analogie de définition entre le corps et l'âme, respectivement, vis-à-vis de la po $\pi$ ク́, dont il résulte plusieurs constats. D’une part, elle incite à considérer l'intellect et l'âme comme des quantités, donc à étendre les limites de cette catégorie. Si l'inclinaison se révèle une espèce de la quantité qui a pour propriété de s'observer dans le mouvement et si l'âme manifeste un tel mouvement d'inclinaison, alors l'âme apparait ellemême comme une quantité. De plus, si le critère sépare dans la quantité ce qui possède une porń de ce qui n'en possède pas, alors l'intellect s'identifie à une quantité, mais dépourvue d'inclinaison. À tout le moins, la conclusion peut surprendre. D'autre part, en indiquant deux orientations pour l'âme-l'une vers le corps, l'autre vers l'intelligible—, l'analogie montre que l'inclinaison peut se produire en deux sens, sans se réduire au seul mouvement de gravité vers le bas. Ce qui vaut pour les incorporels semble également avoir cours parmi les réalités corporelles. L'analogie paraît néanmoins comporter un point de rupture. En effet, tandis que l'âme peut incliner dans l'une ou l'autre direction, selon qu'elle se tourne vers les sensibles ou vers l'intelligible, les corps inclineront toujours dans la direction qui leur convient par nature.

Toutefois, la lecture parallèle des Mystères d'Égypte complète l'analogie. Jamblique y décrit l'âme totale ( $\psi v x \grave{\eta} \dot{\eta}$ ö $\lambda \eta$ ) comme l'âme absolument purifiée des dissonances de la matière et qui a pour type un feu sans tâche ni mélange ${ }^{73}$. Elle est animée d'une lumière intérieure, pure et stable, qui la conduit vers le haut. À l'inverse, incline vers le bas l'âme qui traîne des traces de liens et de châtiments, alourdie par des combinaisons de pneumas matériels et retenue par les troubles de la matière. Le mouvement d'inclinaison de l'âme dépend donc de sa structure physique, selon qu'elle possède une nature purement ignée ou entachée de matière plus grave. À l'idée plotinienne de libre détermination par l'âme de son inclinaison,

73) De Myst., II, 5, 15-29 (édition Des Places 1966). 
Jamblique oppose une théorie de la purification psychique, qui correspond trait pour trait à une purification de sa composition physique: seule peut s'élever l'âme délestée de son ancrage matériel, autrement dit l'âme qui s'est déliée de tout attrait envers la matière et qui a modifié du même coup sa propre constitution. De plus, au premier livre du même traité, Jamblique oppose les propriétés des genres extrêmes:

Àl'un appartient l'être le plus élevé, incompréhensible, supérieur à toute mesure, sans forme en ce sens qu'aucune ne le définit; l'autre est subjugué par l'inclinaison

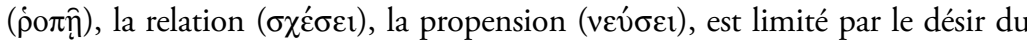
moins bon et la familiarité avec les êtres du second rang, et désormais modelé

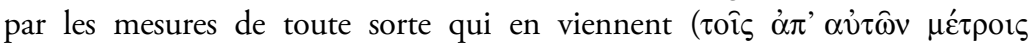

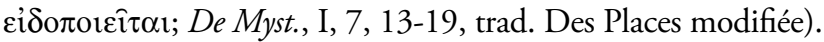

Mêlées au sensible, les réalités inférieures que constituent les âmes incarnées sont soumises à une inclinaison vers le bas, poussées qu'elles sont par un désir du pire. À la différence des réalités supérieures, elles sont déterminées par les mesures que les Formes inscrivent dans la matière. D’une certaine manière, nous pouvons en déduire qu'elles s'apparentent à des formes de quantité, dans la mesure où elles possèdent des limites mensurables. Par conséquent, bien que, dans la citation de Simplicius, Jamblique n'explique pas de quelle façon la porní relève de la quantité, le parallèle esquissé avec les Mystères résout, à tout le moins partiellement, la question de la relation entre quantité et inclinaison psychique.

La citation de Jamblique s'achève sur une question oratoire: pour quelle raison les intervalles de la voix relèveraient-ils de la quantité et pas ceux de la joлń? S'il est possible de distinguer entre syllabes longues et brèves, de chiffrer les rapports musicaux, de parler de rythmes et de mètres, de fixer des limites, pourquoi n'en irait-il pas de la même façon pour cette forme de quantité qu'est l'inclinaison ${ }^{74}$ ? Le découpage qu’opère Simplicius au sein de l'argument se révèle quelque peu étrange, car il passe abruptement de considérations d'ordre hypostatique à une analogie de définition. Sa portée s'éclaire dans la suite du commentaire. La comparaison entre po et $\lambda$ ó $\gamma$ o relève d'une argumentation a fortiori: pour quelle raison hésiter à

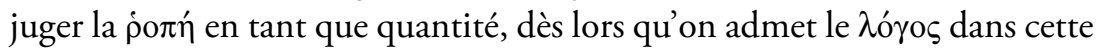

74) Jamblique pense peut-être au Philèbe (17c-e), où Platon rapporte les intervalles sonores à des questions de quantité, exprimées par des rapports numériques. 
catégorie $^{75}$ ? Simplicius mentionne cependant deux objections soulevées contre l'appartenance du $\lambda$ ó ${ }^{\prime}$ as à la quantité $(130,32-131,10)$. En premier lieu, les catégories traitent uniquement des réalités sans liaison, alors

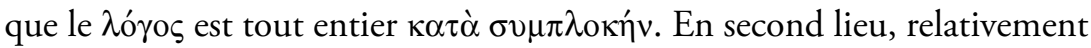
à sa longueur, le $\lambda$ ó tant que $\lambda$ óros signifiant, il reçoit une quantité au vu de l'impact qu'il exerce sur l'air, il devrait plutôt, à cet égard, entrer dans la catégorie de l'agir ou du pâtir, puisque la production d'un son résulte de son action sur l'air-cette seconde objection remonte à Plotin lui-même ${ }^{76}$. Jamblique la résout de la façon suivante $(131,10-17)$ : à la différence d'un doigt, le discours ne produit pas un impact quelconque sur l'air mais engendre une $\varphi \omega v \eta ́$ d'une intensité proportionnée à notre audition. Dès lors, il s'agit d'une action particulière, mesurable et susceptible de variations. En conclusion, si malgré toutes ces difficultés le $\lambda$ ó $\gamma o \varsigma$ appartient à la quantité, la poлń doit à plus forte raison en constituer une espèce, tant il est possible d'établir en son sein des rapports exprimables en termes de quantité, de proportion et de mesure.

La suite de la citation évoque une polémique à l'égard de Porphyre, relative à la doctrine des Catégories. Au sein de son propre Commentaire, Jamblique devait rectifier les propos de son maître vis-à-vis desquels il se trouvait en désaccord. À la faveur de cette discussion, Simplicius dresse un constat essentiel pour envisager le statut de la porń en tant que quantité:

Contre Cornutus et Porphyre qui soutiennent que l'inclinaison observée selon la lourdeur et la légèreté est une qualité, Jamblique affirme que l'inclinaison n'est ni la lourdeur ni la légèreté, mais la mesure de la lourdeur et de la légèreté: "Par eux-mêmes, les lourds ou les légers procéderaient à l'infini s'ils ne possédaient de limite hors d'eux-mêmes. Mais puisque la puissance de l'inclinaison, grâce aux mesures qu'elle leur impose, adjoint un ordre et une limite, ils s'établissent du même coup dans le bon ordre." Comme à l'ordinaire, il traverse et résout de cette façon ces apories relatives au combien $(129,1-7)$.

\footnotetext{
75) Aristote cite le $\lambda$ ó ${ }^{2}$ ○ comme exemple de quantité discrète (Cat., 6, 4 b 23).

76) Plotin, Enn., VI 1 [42] 5, 1-14. Ces objections sont déjà mentionnées par Dexippe, qui cite aussi la solution de Jamblique, mais sur un mode paraphrastique et anonyme (In Cat., 69, 37-70, 8; puis 70, 9-14).
} 
Paraphrasant Jamblique, Simplicius lui attribue une distinction subtile le menant à une double conception de la porń, peu exploitée ici, qui servira plus loin à notre commentateur pour rétablir l'harmonie entre Aristote et Archytas. Dans ces termes, la poñ́ ne désignerait ni la lourdeur ni la légèreté en tant que telles, mais bien la mesure de la lourdeur et de la légèreté. À cet endroit du texte, Simplicius ne discute pas néanmoins cette doctrine jamblichéenne, qui esquisse une démarcation entre la pesanteur envisagée comme qualificatif de la chose et la pesanteur conçue comme sa mesure. Il se contente de l'utiliser comme argument pour asseoir la nécessité de la jo $\pi$ ń en tant qu'espèce de la quantité, soulignant au passage l'efficacité de Jamblique à traverser les apories tout en asseyant sa thèse.

Pour finir, Jamblique esquisse une expérience de pensée proprement irreprésentable pour un néoplatonicien-l'acosmie générale-dans le but de manifester la fonction taxinomique de la porń. À l'intérieur des choses

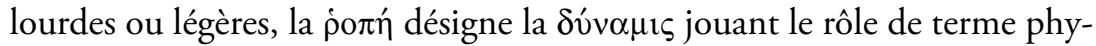
sique qui achève le mouvement et l'empêche de s'effectuer à l'infini. Elle en ressort comme la limite interne au sein d'un corps qui lui impose un ordre. C'est l'expression de sa puissance qui produit le mouvement, selon la mesure qui est la sienne. En vertu de la quantité de ṕotń, l'objet incline vers une place donnée, accomplissant le mouvement rectiligne adéquat

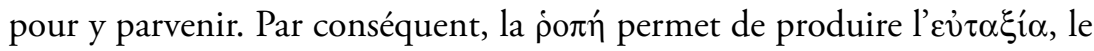
bon ordonnancement du monde où chaque objet occupe la place qui correspond à la mesure de son inclinaison. Elle définit ainsi le critère distinctif entre le monde terrestre et le monde céleste et, parmi les incorporels, entre notre âme et l'Intellect.

\section{La position aristotélicienne}

Dans les Catégories, Aristote n'envisage nulle part la question de la lourdeur et de la légèreté. Pas davantage n’y mentionne-t-il la porń. En d'autres termes, le débat autour du statut de la pesanteur parmi les catégories s'avère postérieur à Aristote lui-même, et tout ce qui pourrait en être dit par les commentateurs résulte d'un croisement de données et d'une reconstruction à partir des leçons des Catégories sur la qualité et sur la quantité, mais aussi d'autres textes du corpus aristotélicien qui abordent la porń, en particulier le traité $D u$ Ciel.

Dans le De Calo, Aristote évoque la porń à plusieurs reprises, dans le cadre de son étude des corps lourds ou légers. Il l'identifie à la tendance 
que manifeste un corps à rejoindre ou à fuir le centre ${ }^{77}$. Elle constitue le principe du mouvement naturel des corps, dans la mesure où elle exprime une propriété inscrite en eux et correspond à ce qui fait qu'un corps se meut, soit à partir du centre soit vers lui. À l'inverse, le corps privé de potń s'avère un corps immobile et mathématique qui, de surcroît, ne peut être considéré comme se trouvant en un lieu ${ }^{78}$. Aussi, la poлń impulse un mouvement naturel, non contraint, en vertu duquel un corps gagne le lieu qui lui est naturellement apparenté et qui fournit la limite de son mouvement $^{79}$. Pour cette raison, Aristote avance, comme entre parenthèses, la définition suivante:

Car nous disons que quelque chose est "pesant" et "léger" par son aptitude à

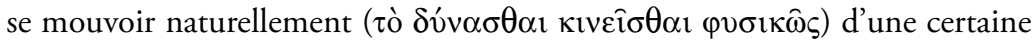

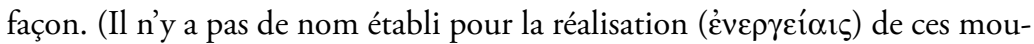

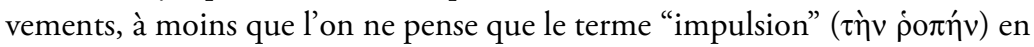
soit un (IV, 1, 307 b 31-34; tr. Dalimier et Pellegrin 2004).)

La роли́ ne serait rien d'autre que l'actualisation du mouvement naturel d'un corps qui rejoint son lieu propre. Dans ces conditions, elle s'avère complètement indépendante de la grandeur et de la petitesse ${ }^{80}$. L'inclinaison d'un corps ne résulte pas de ses dimensions, pas plus que sa pesanteur. Elle découle de sa forme élémentaire: selon l'élément qui le compose, un corps aura tel ou tel mouvement. S'il est fait de terre ou d'eau, il visera le centre; s'il est fait d'air ou de feu, il s'en écartera. Dans le contexte de l'analyse aristotélicienne, la joлtr ne se trouve nullement liée à des considérations quantitatives, mais bien qualitatives. Elle constitue une propriété des corps, définissant le terme de leur mouvement naturel.

En outre, Aristote traite la poлń au moyen du plus et du moins, autrement dit des critères qui servent dans les Catégories à définir la qualité: de deux corps qui ont une $\dot{\rho} о \pi \dot{n}$, qui tendent à gagner le centre, le plus lourd

\footnotetext{
77) Aristote, Du Ciel, II, 14, 297 b 6-7; 12-14.

78) Du Ciel, III, 6, 305 a 24-31.

79) Du Ciel, III, 2, 301 a 22-26.

80) Du Ciel, II, 14, 297 b 7-10: "Peu importe que l'on parle d'une motte de terre, de n'importe quelle partie de la terre ou de la Terre entière. En effet, ce dont il est question n'est pas une conséquence de la petitesse ou de la grandeur, mais est une caractéristique de tout ce qui possède une impulsion vers le centre."
} 
poussera le plus léger dans le mouvement centripète commun ${ }^{81}$. Il s'agit d'une même tendance, c'est-à-dire de la même expression d'une propriété, mais qui se trouve plus ou moins marquée, selon la pesanteur respective. La théorie aristotélicienne de la porń obéit donc résolument à une logique de la qualité-c'est d'ailleurs parmi les espèces de la qualité que, dans la Métaphysique, Aristote range la pesanteur et la légèretée ${ }^{22}$.

Devant le silence criant des Catégories, Simplicius transfere au Stagirite la position du plus célèbre commentateur péripatéticien, Alexandre d'Aphrodise. Digne héritier de la tradition aristotélicienne, Alexandre se doit d'en avoir exprimé le sens avec la plus grande fidélité. Au vu de la théorie de la porń développée dans le De Calo, il ne semble cependant pas avoir trahi l'esprit d'Aristote en intégrant l'inclinaison à la qualité:

Or, en plaçant à la suite d'Aristote l'inclinaison non pas dans le combien, mais dans le quel, Alexandre soutient que l'égal et l'inégal ne s'appliquent pas aux lourds au sens propre, mais de façon abusive. Car c'est le semblable et le dissemblable qui leur conviennent, comme aux autres quels. Le fait d'être mesuré, dit-il, en est bien affirmé de façon assez vague: "En effet, pour le blanc, celui-ci est dit dix fois plus blanc que celui-là, sans qu'il ne soit mesuré par la dixième partie du blanc, mais par la partie de la surface où se trouve le blanc. Or, si le blanc est mesuré par accident, le lourd le sera de la même façon: par le fait de mesurer le corps dans lequel se trouve la lourdeur. Le lourd existerait en vertu de la qualité et non de la grandeur, puisque tout ce qui serait plus lourd serait directement aussi plus grand que le plus léger, du moins serait-il aussi de mesures plus grandes. Or, s'il n'est pas mesuré, l'égal et l'inégal ne se diront pas du lourd ou du léger." Voilà l'argument détaillé d'Alexandre (151, 35-152, 13).

Pour Alexandre, la porń ne désigne une quantité qu'en vertu d'un abus de langage car, en tant que telle, la lourdeur ne fait l'objet d'aucune mesure. C'est plutôt le corps dans lequel elle se trouve qui est mesuré et dont la variation des dimensions modifie la pesanteur: il existe une corrélation

\footnotetext{
81) Du Ciel, II, 14, 297 a 25-30.

82) Aristote, Métaphysique $\Delta$ 14, 1020 b 8-12 (tr. Tricot 1953): "La qualité se dit encore des propriétés des substances en mouvement, comme la chaleur et la froidure, la blancheur

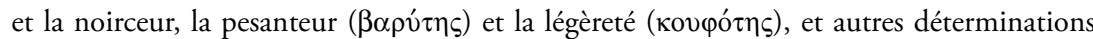
de ce genre, d'après lesquelles, quand elles changent, les corps aussi sont dits subir une altération."
} 
entre les changements de mesure du corps et de sa pesanteur. L'affirmation entre apparemment en contradiction avec la thèse aristotélicienne du De Calo. En réalité, lorsque Aristote postule l'invariabilité de l'inclinaison par rapport à la taille, il entend souligner que la po $\pi$ ń d'un corps résulte de sa nature. La limite de son mouvement s'avère indépendante de ses dimensions. En revanche, pour Alexandre, la taille influence accidentellement la pesanteur: plus un corps est grand, plus de parties de lui-même manifestent sa propriété. Une portion de terre sera dite plus lourde qu'une simple motte, du simple fait de sa plus grande taille. Averti selon toute vraisemblance de l'existence d'un débat sur la nature de la pesanteur, Alexandre se range derrière la position d'Aristote et la consolide au passage. Il n'altère pas radicalement la thèse aristotélicienne mais rend compte d'un fait de langage, inadapté à la théorie des lieux naturels. De la motte à la portion de terre, c'est la même qualité de pesanteur qui s'exprime ${ }^{83}$.

Ce n'est donc pas la poлń elle-même qui apparaît égale ou inégale à une autre, mais le corps où elle se manifeste, qui s'avère ou non égal en grandeur à un corps du même type. Dans ces conditions, il faut uniquement

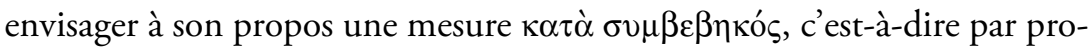
jection d'un constat établi pour le corps dont elle est la propriété. Il est dès lors préférable de traiter la pooń en termes de similitude et de dissemblance, qui sont les propres de la qualité (cf. Cat., 11 a 15-19): la pooń d'un corps est semblable à celle d'un autre, à la façon dont la blancheur d'une surface est comparable à celle d'une autre surface.

Les exégètes plus récents, qui posent conformément à Archytas trois genres du combien, n'affirment pas que la lourdeur est mesurable au moyen du

83) Cette citation nuance, en apparence du moins, la position qu'Alexandre exprime en in Met:: " Mais, à présent, le lourd et le léger se trouvent dans les combien par soi; or Aristote les comptera sous peu parmi les quels. Peut-être seraient-ils des combien d'une part, en tant qu'ils signifient un excès ou un défaut d'inclinaison, des quels d'autre part, en tant qu'ils étaient ce qui possède des causes identiques de se mouvoir vers le bas (397, 21-24). " Sans traiter le double sens avec détail, ce bref passage est pourtant compatible avec celui que discute Simplicius. D'une part, Alexandre parle avec retenue: pour poser que lourd et léger appartiennent à la quantité, il emploie '̌ $\sigma \omega \varsigma$ et le conditionnel, comme s'il rapportait une thèse à l'égard de laquelle il se montre prudent. D'autre part, il opère une différence de niveau, en soutenant que lourds et légers sont des qualités, mais signifient des quantités. Cela revient à dire que, pour lui, seul le sens qualitatif de la ṕotí doit s'entendre proprement, tandis que le sens quantitatif se comprend uniquement de façon dérivée. 
corps, car les mesures du corps et de la lourdeur seraient identiques. Or la mesure du corps et celle de l'inclinaison sont souvent inverses. Par exemple, dans le cas du plomb et de la laine, la mensuration du corps est inégale, s'en trouve-t-il ainsi, tandis que l'inclinaison est égale. Ou, à rebours, les masses seront égales, les inclinaisons inégales. Peut-être que, pour tous les corps, les

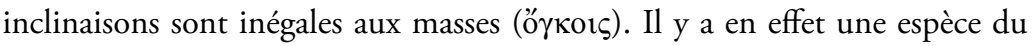
combien dans la grandeur, une autre dans l'inclinaison. La mine et le talent diffèrent spécifiquement du pied et de la coudée, de sorte qu'ils sont mesurés par un autre combien, ne sont pas un quel et ne sont pas contenus dans un des autres combien $(152,13-23)$.

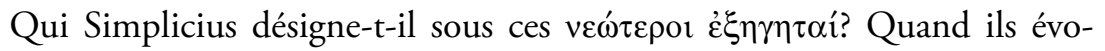
quent la question de la triple division de cette catégorie, le reste des commentateurs s'y montrent hostiles. Seul Ammonius adopte une attitude de réserve: il se contente de la mentionner et d'y joindre les raisons qui servent à la justifier ${ }^{84}$. Son successeur, Olympiodore, prend une position moins favorable, dans la mesure où il se prononce ouvertement contre la possibilité de traiter la jo ń $_{\text {comme une espèce à part entière de la quantité }}^{85}$. Élias suit une trajectoire similaire et poursuit la critique: il ne faut pas considérer la jo comme un $\pi \alpha ́ \theta$ os de la quantité ${ }^{86}$. En substance, l'objection revient à la

\footnotetext{
84) Ammonius, In Cat., 55, 4-10: "Certains affirment que les espèces au sens propre du

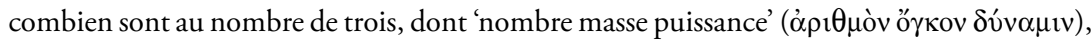
c'est-à-dire l'inclinaison. Ils soutiennent que le discours et le temps sont identiques au nombre; que la ligne, la surface et le corps peuvent être ramenés à quelque chose de commun, la grandeur; que le lieu est identique à la surface; de sorte que, de la quantité, une espèce est le nombre, l'autre la grandeur, la troisième la puissance. Sous cette dernière peuvent être ramenés le lourd et le léger, qui sont des inclinaisons relevant du combien."

85) Olympiodore, In Cat., 82, 33-40: "Aristote divise le combien en continu et en discret. On objecte directement à cette division qu'elle ne circonscrit pas toutes les espèces: l'inclinaison, tiens, qui est une espèce du combien (en tant que nous parlons du talent, du demi-talent), est laissée de côté. À cela, certains répondent que l'inclinaison est identique au continu et que, pour cette raison, Aristote ne l'a pas mentionnée. Quant à nous, nous répondons que c'est faux: $\dagger \ldots$ car on déclare ces choses de même masse ou de même lourdeur. Que répondons-nous donc? Que l'inclinaison n'est pas une espèce du combien, mais plutôt une affection $(\pi \alpha \dot{\theta} \theta$ os) du combien, tout comme le pair et l'impair ne sont pas des nombres, mais des affections du nombre."

86) Élias, In Cat., 186, 21-32: "Nous disons que l'inclinaison ne peut constituer une troisième espèce du combien, puisqu'elle est une affection du combien continu. Tout corps est en effet soit léger soit lourd. Comme la chaleur est une qualité essentielle dans le feu,
} 
thèse soutenue par Alexandre d'Aphrodise: la poлń concerne seulement le corps qualifié et non le corps comme tel. Ajoutons que seul Élias attribue la paternité de cette triple division à Archytas, citant aussi le nom de Ptolémée $^{87}$. De plus, il mentionne Platon à leurs côtés, auquel il impute également cette doctrine ${ }^{88}$.

Au vu de la vive hostilité que suscite la tripartition de la quantité auprès des commentateurs alexandrins, il ne reste guère plus que Jamblique et, peut-être, Syrianus parmi les candidats possibles pour identifier les interprètes récents ${ }^{89}$. Contre Alexandre mais à la suite d'Archytas, ils ont distingué mesure du corps et mesure de la lourdeur. Leur argument vise à rendre raison du donné empirique: à inclinaison identique, la laine et le plomb manifestent des dimensions différentes, et inversement. En réalité, les deux camps cherchent la solution de problèmes distincts. Les aristotéliciens-rejoints à certains égards par les commentateurs alexandrinsrendent compte de l'identité du mouvement centripète de deux corps de même nature. Suivant Archytas, les platoniciens s'interrogent sur les écarts de pesanteur entre des corps de natures différentes. Cette manière différente d'envisager la question aboutit à une réponse originale, qui utilise aussi l'argument de l'indépendance de la grandeur et de la petitesse à l'égard de

l'impair et le pair † sont des qualités essentielles dans le nombre, la lourdeur et la légèreté † sont des qualités essentielles dans le corps, du fait que le corps dans sa totalité est soit lourd, soit léger. En outre, il faut montrer que la légèreté et la lourdeur ne sont pas des espèces du combien, mais bien une affection, puisque le discret s'observe directement dans les réalités divines, immatérielles et intelligibles, où il n'y a pas d'inclinaison. Premièrement, donc, le discret est une espèce du combien; deuxièmement, en est une autre l'opposé au discret, à savoir le continu - la ligne, la surface, le corps. Or rien de tel n'est léger ou lourd, ni la ligne, ni la surface, ni le corps mathématique, ni le corps sans qualité, mais seulement le corps qualifié. Les inclinaisons sont donc des affections, et non des combien au sens propre." La restitution entre cruces vise à combler une lacune due à un saut du même au même de la part d'un copiste.

87) Élias, In Cat., 185, 9-10: "Troisièmement, il faut examiner si ce genre comporte ces espèces seules [discret et continu], ou bien aussi l'inclinaison, comme l'affirment Platon, Archytas et l'astronome Ptolémée."

88) Signalons que Dexippe est muet sur ce point. Quant à Philopon, dans son Commentaire sur les Catégories, il traite seulement de la ṕo $\eta$ dans un registre moral: la puissance

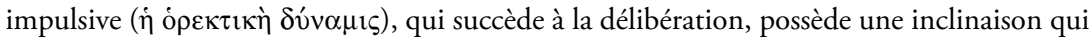
mène l'action dans l'une ou l'autre direction (In Cat., 146, 4-6).

89) Dans l'In de Calo, Simplicius identifie explicitement Jamblique, en une occasion du

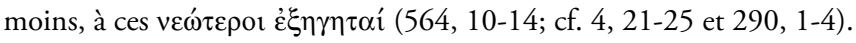


la pesanteur, mais en vue d'un tout autre constat et au vu d'une tout autre question: comment expliquer les variations d'inclinaison qui affectent des corps différents?

La $\dot{\rho} \pi \eta \dot{~ s e ~ r e ́ v e ̀ l e ~ b i e n ~ u n e ~ m e s u r e ~ a u t o n o m e ~ v i s-a ̀-v i s ~ d e ~ l a ~ g r a n d e u r . ~}$ Elle possède d'ailleurs un système métrique propre, différent de celui utilisé pour les autres quantités: par exemple, la mine et le talent sont indépendantes du pied et de la coudée. Si devant l'incommensurabilité à l'égard des autres quantités la jo $\pi$ ń s'avère une espèce à part entière, elle doit posséder une différence qui exprime sa particularité. Or Simplicius a rappelé que la division aristotélicienne entre grandeur et nombre reposait sur deux différences: le continu pour la grandeur, le discret pour la pluralité $^{90}$. Dès lors, il reste à énoncer la différence correspondant à la dernière espèce de quantité. Malheureusement, ni Archytas, ni Jamblique, ni Simplicius ne la fournissent.

À bien y réfléchir, c'est-à-dire dans ce cas à examiner l'exemple choisi par Simplicius, cette troisième différence ne peut aboutir qu'à un seul résultat: le dense. Si la jo dimensions des corps_comme l'indique l'exemple du plomb et de la laine-, le critère de variation doit provenir de la constitution interne de ce corps, en d'autres termes de la quantité de matière qui le compose. L'observation montre que le plomb est plus compact que la laine: ses parties apparaissent plus solidaires et plus serrées les unes aux autres. Il possède une densité supérieure. Par conséquent, la division triple avancée par Archytas, reprise par Jamblique et Simplicius, fait apparaître l'idée de densité, qui pourrait fournir le troisième critère de la quantité, à côté du continu et du discret. Dans ces conditions, le poids s'avère bien indépendant de la taille du corps et justifie une troisième espèce.

Avec ce troisième critère, du moins avec l'affirmation d'une troisième espèce de la quantité, la physique prend une tout autre tournure. Si les corps n'obéissent plus à une propriété inscrite naturellement en eux mais suivent un mouvement en vertu de la quantité de matière dont ils sont composés, la physique se met en chemin vers une conception plus moderne, qui cherche moins à comprendre les similitudes entre des corps

90) In Cat., 123, 1-15. Dans l'exposé d'Aristote toutefois, ces différences apparaissaient comme les véritables espèces, alors que nombre et grandeur constituaient des entités leur appartenant (Cat., 6, 4 b 20). 
de même nature que les rapports entre des corps de natures différentes. Il ne s'agit certes là que d'une première étape. Le vocabulaire moderne, en effet, assortira la densité à la masse, c'est-à-dire à la quantité de matière qui compose le corps: un corps plus dense sera aussi d'une masse plus élevée.

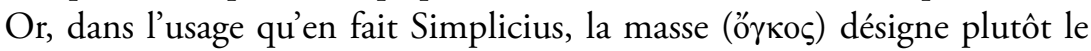
corps en tant que mesurable, selon sa grandeur. Il restera à se former un lexique adapté.

Mais Alexandre, présumant pour je ne sais quelle raison ce qui est au début (que le mesuré est seulement selon la grandeur, mais pas selon l'inclinaison), conclut que l'inclinaison n'est pas un combien, mais un quel. Or, si le lourd différait du lourd d'après la qualité, la variation se produirait grâce à des différences de caractères, comme cela s'observe pour toutes les autres choses qui varient par des qualités. En réalité, l'inclinaison, en tant qu'elle est inclinaison, possède la même particularité, et elle ne varie que parce qu'elle entraîne telle ou telle quantité, selon le rapport de convergence vers le centre. Il y a donc trois espèces du combien et c'est de trois façons qu'on observe l'égal et l'inégal dans ces réalités (152, 23-31).

La surprise qu'exprime Simplicius à l'égard des absurdités auquel aboutit Alexandre souligne sa défiance par rapport à ce commentateur qu'il juge d'habitude si brillant et pour lequel il éprouve par ailleurs le plus profond respect. Du point de vue de la méthode exégétique, il en ressort qu’à une foi aveugle, Simplicius privilégie un changement d'autorité, s'il s'en trouve une en meilleur accord avec le donné empirique: exégète informé et fidèle, il ne s'occupe pas moins de rendre raison de la réalité.

Notre commentateur énonce enfin la propriété de la porń, qui est d'exercer une attraction de telle ou telle intensité. Celle-ci varie en fonction d'un calcul de convergence vers le centre de gravité, et non d'une différence de caractère (qui serait une propriété qualitative). La pression que l'objet lourd suscite sur la balance est chiffrable et comparable à celle d'un autre, à l'aide du système des poids. Dès lors, si un corps tend à rejoindre le centre, ce n'est plus simplement parce qu'il vise à gagner son lieu naturel, mais parce que réside en lui une quantité déterminée d'attraction. Et à partir du moment où il devient possible de chiffrer un tel mouvement, nous quittons la sphère d'une physique régie par des principes téléologiques, pour entrer dans une physique plus mécaniste, où les différences de mesure entraînent des différences d'effet. 
Pour conclure sur cette divergence entre Aristote et ses interprètes néoplatoniciens tardifs, il semble que la différence de conception physique résulte d'un déplacement du regard, passant de la recherche de ce qui assure l'identité à ce qui justifie les différences. Loin d'être anodin, le changement de perspective aurait pu initier le passage à la physique moderne.

\section{La conciliation}

La constitution d'une troisième espèce de la quantité confronte Simplicius à un dilemme. Si la division d'Aristote s'avère erronée et inadéquate à l'égard du réel, faut-il conclure à la mise au rebut de sa théorie physiqueet, par extension, de la logique qui la sous-tend? Ou bien, si le travail du bon exégète consiste à chercher une solution aux problèmes de divergence, ne convient-il pas d'en chercher la raison et, mieux encore, de trouver un moyen pour restaurer l'harmonie? C'est ce second parti que prend Simplicius, à l'intérieur cette fois de l'examen des espèces de la qualité (sur Cat., 8,10 a 11 s.):

$\mathrm{Vu}$ que certains ont introduit à cet endroit le propos sur la pesanteur et la légèreté, il est nécessaire de les parcourir quelque peu et d'affirmer que le

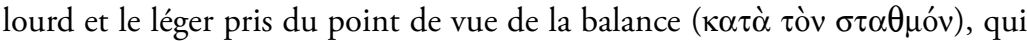
recourent pour l'inclinaison au plus et au moins, seraient un combien, étant donné qu'Archytas aussi a défini une espèce une du combien relative à l'inclinaison et à la balance. En revanche, les propriétés à l'entour des corps, en fonction desquelles l'air est dit léger et le feu plus léger que l'air, la terre est dite lourde et plus lourde que l'eau, celles-ci manifestent une qualité. De la même façon que le fin et l'épais, ceux qui sont dans la masse et mesurés

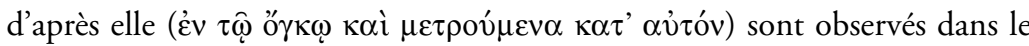

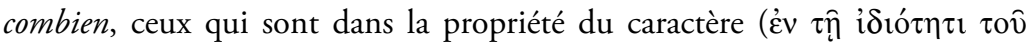

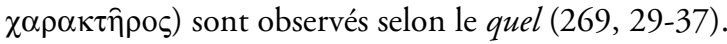

Le point est désormais établi et Simplicius ne reprend plus sa démonstration: la poлń relève de la quantité, conformément à la division archytéenne. Or se conformer à la seule position du Pythagoricien reviendrait à ruiner une partie de l'édifice logique des Catégories, mais aussi—et surtout—de la cosmologie qualitative à la base du De Calo. Dès lors, bien qu'il s'accorde avec Jamblique pour reprendre l'institution par Archytas d'une troisième espèce du combien, relative au poids, Simplicius se montre également 
attentif à une nuance logée dans le texte de son prédécesseur. Dans ses termes, il faudrait distinguer entre la pesanteur et la mesure de la pesanteur (In Cat., 129, 1-4.). Se dessine une différence entre aspects qualitatif et quantitatif. C'est la voie que notre exégète emprunte pour concilier les théories d'Archytas et d'Aristote, en opérant une distinction au sein de la ролт́ qui aboutit à deux idées de la pesanteur.

Selon la première, il existerait une forme de $\dot{\rho} 0 \pi \hat{n}$ correspondant à la

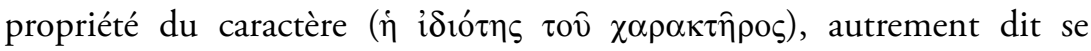
situant au niveau de ce qui fait qu'une réalité est telle qu'elle est. Elle équivaudrait à la qualité essentielle associée aux éléments, telle la légèreté du feu ou la lourdeur de l'air, désignant la propriété d'un corps à incliner plus ou moins et découlant de sa nature élémentaire—puisque, conformément à la cosmologie aristotélicienne, chaque élément possède une propriété. Notons au passage que Simplicius ne mentionne, à propos de ce premier sens, que les éléments en tant que tels: le feu, l'air, la terre et l'eau. Veut-il suggérer que cette forme de poлń ne concerne que les éléments, auxquels serait assortie une qualité particulière (lourdeur ou légèreté)? Ou bien sousentend-il que, selon la concentration des éléments présents en lui, un corps possèdera une po $\pi$ ń plus ou moins élevée? La solution à cette alternative de Simplicius est à trouver dans la recherche de l'espèce de la qualité à laquelle devrait être rapportée la poлń-en un sens qualitatif cette fois.

Donc, parmi ceux-ci, tout ce qui n'est pas coordonné au combien, mais au caractère et au quel, est-ce qu'on les range dans une autre espèce de la qualité, à côté des quatre citées, comme Andronicos et Plotin le comprennent, ou bien est-il possible de les faire entrer sous l'une des quatre? Cela deviendra clair quand on saura, au sujet de la lourdeur et de la légèreté mêmes, ce qu'elles sont. Car, si la chaleur, qui déploie les corps, produit le léger identique à elle-même et si la fraîcheur, qui les contracte, produit le lourd identique à elle-même, ils seront ramenés à des qualités affectives. Mais si la légèreté accompagne le chaud, la lourdeur le froid, la chaleur et la fraîcheur seront aussi des qualités actives. Or le lourd et le léger ne feront ni ne subiront rien par nature, mais les premières sembleront ramenées à des qualités affectives, les secondes à un autre genre propre. Et si elles appartiennent par essence aux éléments, elles ne sembleront pas être des qualités. Tandis que si leur réceptacle est et est dit un quel, et si à partir de la différence essentielle

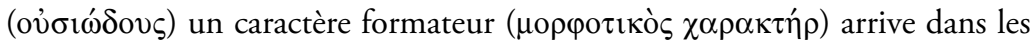
corps participants, ceux-ci seront aussi des quels (269, 37-270, 14). 
À l'instar du sens quantitatif, faut-il soit considérer que la po $\pi$ ń qualitative forme une espèce autonome, comme l'ont estimé Plotin (VI 1 [44] 11, 29-32) et Andronicos, soit l'intégrer à l'une des quatre autres espèces de la qualité? Si le froid produit le lourd par contraction tandis que le chaud produit le léger par dilatation, alors il devrait s'agir de qualités affectives, au sens où lourd et léger résulteraient d'une affection-d'une action exercée sur eux par la chaleur ou la fraîcheur. Or, dans les Catégories, les qualités affectives, auxquelles appartiennent la chaleur et la fraîcheur, ne sont pas dites affectives du fait que les corps qui en sont pourvus en seraient euxmêmes affectés, mais parce que ces qualités sont susceptibles de produire une affection, au gré des sens: au toucher, la chaleur produit une certaine affection, comme la douceur produit au goût une affection donnée (Cat., 8, 9 b 3-9). Par conséquent, puisque le lourd et le léger ne produisent pas ce type d'affection mais seraient eux-mêmes le fruit d'une affection, ils doivent être reconduits vers une espèce de la qualité différente des qualités affectives, ainsi que des états et dispositions, des capacités naturelles et des qualités dérivées. Simplicius se rallie sur ce point à l'avis de Plotin.

Lourd et léger ne peuvent pas non plus simplement s'identifier aux propriétés essentielles des éléments: ils ne constitueraient plus dans ce cas des qualités mais relèveraient de la nature même des éléments. Au contraire, ils altèrent le réceptacle qui les accueille, font en sorte qu’il soit qualifié et dit d'une certaine façon. Ils lui impriment donc une différence essentielle en vertu de laquelle un élément est informé et se distingue des autres: c'est par sa lourdeur, sa froideur et sa contraction que la terre se distingue du feu, plus étendu, plus chaud et plus léger. L'inclinaison est qualité en ce que la participation aux éléments dans lesquelles elle siège déterminera la formation des corps, c'est-à-dire engendrera leur pesanteur. Dès lors, cette forme de poлń peut être en quelque sorte rapportée à la notion de masse volumique, au sens où elle désigne une propriété attachée intrinsèquement à un objet en fonction de sa constitution, en vertu de laquelle il est dit, par nature, plus ou moins lourd.

Selon la seconde signification, en tant qu'elle se trouve dans une masse et est mesurée, la poлń désigne la quantité en fonction de laquelle un corps possède une puissance plus ou moins élevée d'attraction vers le centre. Quelle relation entretient-elle avec la première forme de poлń? Si les éléments possèdent une pesanteur en vertu d'une détermination qualitative et si les corps sont faits des éléments, alors la ṕotń d'un corps envisa- 
gée quantitativement correspondrait à sa possession plus ou moins élevée des caractères élémentaires. C'est du fait que les corps sont constitués

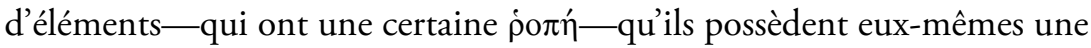
ค่оли́ donnée. Nous en revenons à l'idée de densité: le corps plus lourd contiendra davantage d'éléments contractés, le corps plus léger davantage d'éléments étendus.

Après avoir emboîté le pas à Jamblique et à Archytas pour défendre la valeur de la $\rho$ ón en tant que quantité, Simplicius déploie à présent autant d'arguments pour l'identifier en tant que qualité. Cependant, la seconde démarche ne vient pas annuler la première. Elle la complète en produisant deux notions distinctes de la pesanteur, qui rétablissent l'harmonie entre Archytas et Jamblique d'un côté, Aristote et Plotin de l'autre, par l'attribution d'une vérité à chacune de ces théories. Elle distingue simplement les propos: la jo $\pi$ ń comprise comme quantité renvoie à la pesanteur des corps, tandis que la jo

\section{Vers une physique quantitative?}

La distinction d'une troisième espèce de quantité, qui apparaît dans le traité Sur les Notions universelles d'Archytas, trouve en Jamblique et en Simplicius des lecteurs intéressés. Elle est aussi le signe qu'au tournant du $\mathrm{I}^{\text {er }}$ siècle $\mathrm{ACN}$ et du début de notre ère, la physique éprouvait le besoin d'outils quantitatifs supplémentaires. Elle répond en effet à un constat empirique: si les balances nous permettent de mesurer le poids des corps, il doit dès lors exister une forme de quantité qui corresponde à ce phénomène, incommensurable au nombre ou à la grandeur. Nous avons là la trace qu'à un moment de l'histoire des sciences s'est ouverte, dès l'Antiquité, la possibilité de passer d'une physique qualitative à une physique quantitative.

Sur cette question de la pesanteur, comme sur beaucoup d'autres, Simplicius emprunte la solution de Jamblique_elle-même inspirée d'Archytas. Il le suit pour instaurer une troisième espèce de quantité, qui puisse rendre compte des différences de poids. Toutefois, il s'en détache quelque peu quand il s'agit de restaurer un plus haut degré d'harmonie, en la rétablissant non seulement avec les réalités empiriques, comme le soulignait déjà Jamblique, mais également entre les autorités: Archytas et Aristote. Cet exercice le conduit à produire une nouvelle doctrine-aussi inédite qu'en contradiction avec d'autres commentateurs des Catégories (Alexan- 
dre, Olympiodore, Élias)—, qui distingue entre deux niveaux de la po $\pi$ ń et manifeste une différence entre la propriété des éléments et celle des corps qui en participent, où apparaît la possibilité d'une mesure.

Cependant, l'innovation survient chez Simplicius sans préparation. La forme quantitative du poids n'apparaît en effet pas de façon nette dans le reste des Commentaires. Il y est souvent question de la porń en tant que principe du mouvement d'inclinaison vers le centre, mais n'y est envisagée aucune possibilité de mesurer ce mouvement. La poлń reste plutôt associée à une propriété élémentaire ${ }^{91}$. Tout en étant le témoin de la production d'une nouvelle physique et tout en produisant lui-même une nouvelle doctrine, Simplicius reste par ailleurs l'héritier d'une tradition, qui s'est poursuivie bien après lui.

\section{Conclusion}

Sans lui consacrer de Commentaire autonome, Simplicius compte Archytas parmi les auteurs qu'il suit de près. Dans son interprétation des Catégories, il lui arrive même d'utiliser le traité Sur les notions universelles comme support: à propos des catégories finales qu'Aristote lui-même évoque à peine, Archytas sert de base à l'exégèse, à laquelle il fournit les définitions, les propres et les divisions. Pour ce faire, en pédagogue consciencieux, Simplicius formule la ligne de conduite pour mener en parallèle la lecture des deux textes, en sorte que l'un vienne pallier les défauts de l'autre. Cependant, le décalage chronologique provoqué par la croyance en l'authenticité du pseudépigraphe le contraint à de nombreuses contorsions dans sa pratique exégétique. Comme derrière toute déclaration d'intention préalable au travail philosophique, il apparaît que les deux auteurs résistent à cette tentative de mise en système univoque et que Simplicius doit s'accommoder d'une certaine dose de bricolage (avec toute la mauvaise foi que

\footnotetext{
91) Sur la centaine d'occurrences de po $\pi$ ń que l'In de Calo, aucune ne renvoie à une signification quantitative. 'Poлń et $\varphi v ́ \sigma ı \varsigma$ y sont souvent liées, Simplicius parlant "d'inclinaison naturelle" (par exemple 258, 2; 286, 21; 693, 13; 693, 21; 700, 18) ou de "nature et inclinaison des corps" (par exemple 263,18). Souvent aussi la po le lieu propre ou naturel $(286,7 ; 712,2)$. Il en va de même dans l'In Phys.: si les occurrences sont un peu moins nombreuses, elles n'en restent pas moins attachées à la dimension qualitative.
} 
comporte ce type d'exercice, auquel nous nous soumettons tous). Il en ressort que:

1) S’il leur attribue un but commun, Simplicius élabore une interprétation d'Archytas qui s'interroge sur les notions universelles dans leur pureté, au lieu d'étudier les catégories en tant qu'elles désignent les réalités (comme le ferait Aristote). Dans ces termes, le traité d'Archytas s'avère plus ontologique que logique.

2) La destination exacte du traité d'Archytas reste discutée. À l'instar des Catégories, il paraît posséder une finalité introductive, qui n’ouvre pourtant plus à la philosophie en général, mais à son sommet seulement: l'ontologie pythagoricienne. Pour cette raison, il renferme un propos plus précis, plus concis et plus complet, dont le déploiement fait la pleine lumière sur des points demeurés obscurs dans le texte d'Aristote.

3) Les hésitations autour de l'ordre conduisent Simplicius à battre en brèche par rapport à son postulat fondamental de la nécessité d'une déduction des catégories. À nouveau, l'ordre observé par Archytas résulte d'une perspective plus élevée dans l'intellection, tandis que les Catégories obéissent à une description empirique de la réalité sensible.

4) Archytas constitue une source capable de légitimer un propos d'Aristote mis en cause par une objection. Néanmoins, Simplicius ne se contente jamais d'un simple argument d'autorité. Il l'agrémente à chaque fois du raisonnement ou de la preuve empirique qui le corrobore.

5) Les désaccords conduisent parfois Simplicius à faire preuve d'inventivité et à élaborer des doctrines originales, qu'il s'agisse de définir la réalité de la relation ou de formuler ses critères propres par rapport à

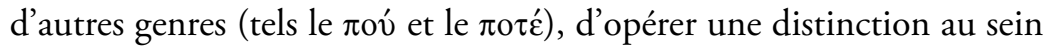
de la notion de masse entre une notion quantitative et une notion qualitative, ou encore de décrire le processus d'inscription de la figure dans les corps. En chacune de ces occasions, Simplicius avance une théorie nouvelle digne d'intérêt pour l'historien de la philosophie.

6) Simplicius utilise plusieurs stratégies d'harmonisation: la distinction entre les niveaux de lecture, la juste compréhension d'un silence, voire la dénégation des écarts. Toutes servent un seul et même objectif, révéler la vérité unique et commune, latente derrière le texte d'Aristote, d'Archytas, mais aussi de Platon, de Plotin et de Jamblique. 
7) De tout cela, il résulte qu'Archytas contribue à la rédaction d'un commentaire capable d'élever l'âme de son lecteur à un niveau plus élevé du réel, réalisant ainsi la véritable tâche du travail exégétique: interpréter le texte afin de mener le disciple sur le chemin de son amélioration psychique.

Simplicius suit certes des modèles, mais son souci de rester fidèle à ses autorités le conduit à s'engager dans des constructions doctrinales inédites, parfois fécondes. Quand il commente une œuvre, il vise non seulement à l'expliquer, mais aussi à révéler la vérité qu’y a exprimée son auteur. À cette fin, il se sert d'outils extérieurs au texte source, dont il cherche également à rendre compte. Or, en bon exégète et pédagogue attentif, il entend en outre rendre leur vérité accessible. Animé d'une conscience aiguë de sa tâche de commentateur, il cherche à mettre le lecteur en contact avec cette vérité, en adaptant certes le propos à son degré d'apprentissage, mais surtout en insistant sur son universalité. Sur bien des points, il se comporte à l'égard de ses autorités comme un bricoleur, s'ingéniant à rétablir l'harmonie profonde de la pensée, reflet de l'harmonie fondamentale de l'univers. Mais les bricolages ne constituent-ils pas ce qui permet à la pensée de progresser et de promouvoir de la nouveauté?

\section{Bibliographie des ouvrages cités}

Aubry, G. 2004. Plotin. 53. I, 1. Introduction, traduction commentaire et notes. Paris: Cerf.

Bodéüs, R. 2001. Aristote. Catégories. Introduction, texte, traduction française et notes. Paris: Les Belles Lettres.

Brisson, L. 2000. "Le commentaire comme prière destiné à assurer le salut de l'âme. La place et le rôle des Oracles Chaldä̈ques dans le Commentaire sur le Timée de Platon par Proclus", in M.-O. Goulet-Cazé (éd.), Le Commentaire entre tradition et innovation. Actes du Colloque international de l'Institut des Traditions textuelles, Paris et Villejuif, 22-25 septembre 1999. Paris: Vrin, 329-353.

Baltussen, H. 2008. Philosophy and Exegesis in Simplicius. The Methodology of a Commentator, London: Duckworth.

Chantraine, P. 1999. Dictionnaire étymologique de la langue grecque, Paris: Klincksieck.

Dalimier, C.; Pellegrin, P. 2004. Aristote: Du Ciel. Texte, traduction française et notes. Paris: Garnier Flammarion.

Des Places, E. 1966. Jamblique. Les Mystères d'Égypte. Paris: Les Belles Lettres. 
Golitsis, P. 2008. Les Commentaires de Simplicius et de Jean Philopon à la Physique d'Aristote, Berlin-New York: De Gruyter.

Hadot, I. 1990. Simplicius. Commentaire sur les Catégories, Fasc. I: Introduction, première partie (p. 1-9,3 Kalbfleisch); traduction de Ph. Hoffmann, commentaire et notes à la traduction par I. Hadot. Leiden-New York-København-Köln: Brill.

- 2001. Simplicius. Commentaire sur les Catégories d'Aristote. Chapitres 2-4. Traduction par Ph. Hoffmann, commentaire par C. Luna. Paris: Les Belles Lettres.

Hoffmann, Ph. 1980. "Jamblique exégète du pythagoricien Archytas: trois originalités d'une doctrine du temps". Les Études philosophiques, 3, 307-323.

— 1987. "Catégories et langage selon Simplicius_la question du skopos du traité aristotélicien des Catégories", in I. Hadot (éd.), Simplicius. Sa vie, son æuvre, sa survie. Actes du colloque international de Paris (28 sept.- ${ }^{\text {er }}$ oct. 1985). Berlin-New York: De Gruyter, 61-90.

— 2000. "Les Catégories aristotéliciennes $\pi$ o $\tau \grave{\varepsilon}$ et $\pi$ oú d'après le commentaire de Simplicius", in M.-O. Goulet-Cazé (éd.), Le Commentaire entre tradition et innovation. Actes du Colloque international de l'Institut des Traditions textuelles, Paris et Villejuif, 22-25 septembre 1999. Paris: Vrin, 355-376.

Huffman, C.A. 2005. Archytas of Tarentum. Pythagorean, Philosopher and Mathematician King. Cambridge: Cambridge University Press.

Moraux, P. 1973. Der Aristotelismus bei den Griechen, t. II, Berlin: De Gruyter.

Szlezák, Th.A. 1972. Pseudo-Archytas über die Kategorien, Berlin-New York: De Gruyter.

Thesleff, H. 1961. An Introduction to the Pythagorean Writings of the Hellenistic Period, Åbo: Åbo Akademi.

1965. The Pythagorean Texts of the Hellenistic Period. Åbo: Åbo Akademi.

Tricot, J. 1953. Aristote. Métaphysique. Traduction française et notes. Paris: Vrin. 